\title{
Incidence of Serious Infections and Design of Utilization and Safety Studies for Biologic and Biosimilar Surveillance
}

\author{
Jie Zhang, PhD; Gayathri Sridhar, PhD; Charles E. Barr, MD, MPH; Bernadette Eichelberger, PharmD; \\ Catherine M. Lockhart, PharmD, PhD; James Marshall, MPH; Jerry Clewell, PharmD, MBA; \\ Neil A. Accortt, PhD; Jeffrey R. Curtis, MD, MS, MPH; Cynthia Holmes, PharmD, MS; \\ Cheryl N. McMahill-Walraven, PhD; Jeffrey S. Brown, PhD; and Kevin Haynes, PharmD, MSCE
}

\begin{abstract}
BACKGROUND: There is a need for postmarketing evidence generation for novel biologics and biosimilars.

OBJECTIVE: To assess the feasibility, strengths, and limitations of the Biologics and Biosimilars Collective Intelligence Consortium (BBCIC) Distributed Research Network (DRN) to examine the utilization and comparative safety of immune-modulating agents among patients with autoimmune diseases.

METHODS: We conducted a retrospective cohort study among patients enrolled in health insurance plans participating in the BBCIC DRN between January 1, 2006, and September 30, 2015. Eligible patients were adult ( $\geq 18$ years) new users of a disease-modifying nonbiologic and/or biologic agent with a prior diagnosis of rheumatoid arthritis (RA), other inflammatory conditions (psoriasis, psoriatic arthritis, ankylosing spondylitis), or inflammatory bowel disease (IBD). Follow-up started at treatment initiation and ended at the earliest of outcome occurrence (serious infection); treatment discontinuation; or switching, death, disenrollment, or end of study period. The study leveraged the FDA Sentinel System infrastructure for data management and analysis; descriptive statistics of patient characteristics and unadjusted incidence rates of study outcomes during follow-up were calculated.
\end{abstract}

RESULTS: Eligible patient drug episodes included 111,611 with RA (75\% female), 61,050 with other inflammatory conditions ( $51 \%$ female), and 30,628 with IBD ( $52 \%$ female). Across all 3 cohorts, approximately half of the patient drug episodes initiated a biologic ( $50 \%$ in RA; $60 \%$ in psoriasis, psoriatic arthritis, ankylosing spondylitis; and $55 \%$ in IBD). The crude incidence rate of serious infection was 9.8 (9.5-10.0) cases per 100 personyears in RA, 7.1 (6.8-7.5) in other inflammatory conditions, and 14.2 (13.614.8) in IBD patients.

CONCLUSIONS: This study successfully identified large numbers of new users of biologics and produced results that were consistent with those from earlier published studies. The BBCIC DRN is a potential resource for surveillance of biologics.

J Manag Care Spec Pharm. 2020;26(4):417-90

Copyright $\odot 2020$, Academy of Managed Care Pharmacy. All rights reserved.

\section{What is already known about this subject}

There is a need for postmarketing evidence generation for novel biologics and biosimilars.

The Biologics and Biosimilars Collective Intelligence Consortium (BBCIC) is a multistakeholder nonprofit public service initiative dedicated to providing scientific evidence on the comparative safety and effectiveness of biosimilars and novel biologics.

The BBCIC Distributed Research Network (DRN) consists of U.S. commercial health plans that participate in the FDA Sentinel System, thus enabling BBCIC to conduct claims-based research specific to biologics using the Sentinel common data model and analytic tools.

\section{What this study adds}

This study demonstrates the strengths of the BBCIC DRN for active surveillance of biologics.

This study identifies methodologic challenges and limitations of the BBCIC DRN and develops recommendations for improving the design and conduct of future comparative safety/effectiveness studies of biologics.

工 n March 2015, the U.S. Food and Drug Administration (FDA) approved the first biosimilar (filgrastim-sndz, Zarxio) for all indications of the reference biologic. ${ }^{1}$ As of January 2019, the FDA has approved 17 biosimilars, which include biosimilars for widely used biologics such as adalimumab (Humira), etanercept (Enbrel), and infliximab (Remicade) that are indicated for the treatment of several autoimmune inflammatory conditions. ${ }^{2,3}$ The availability of biosimilars offers physicians and patients choice in biologic therapies, with potential for cost savings, even though actual savings are contingent on numerous factors and are yet to be evaluated empirically in the United States. ${ }^{4,5}$

All FDA-approved biosimilars are required to be "highly similar" and have "no clinically meaningful differences from the reference product in terms of safety, purity, and potency (safety and effectiveness)."6 However, the population included in pre-approval clinical trials may not be large or heterogeneous enough to identify and assess rare but potentially serious adverse effects. Therefore, it is important to engage in 
postmarketing safety monitoring and evaluation of approved biologics. ${ }^{7}$ Further, the evidence generated through postmarketing comparative utilization and safety studies of biosimilars and reference biologics may help ensure the safety and effectiveness of a biosimilar and instill health care provider and patient confidence in these products. ${ }^{8,9}$

The Biologics and Biosimilars Collective Intelligence Consortium (BBCIC) is a nonprofit public service initiative dedicated to providing scientific evidence on the comparative safety and effectiveness of biosimilars and reference biologics, with participants from managed care organizations, integrated delivery systems, pharmacy benefit management firms, research institutions, and pharmaceutical companies. ${ }^{10,11}$ The BBCIC's Distributed Research Network (DRN) consists of geographically diverse U.S. commercial, including Medicare Advantage, health plans that also participate in the FDA Sentinel System, thus enabling BBCIC to conduct claims-based research specific to biologics using the Sentinel common data model and analytic tools. ${ }^{12,13}$

To evaluate the potential capability of the BBCIC DRN for future study of biosimilar use and safety, we conducted a study among patients with select autoimmune conditions that are commonly managed with anti-inflammatory biologics, such as anti-tumor necrosis factor (TNF) alpha antagonists. ${ }^{14-19}$ These biologics reduce disease activity and systemic inflammation, improve quality of life, and, when initiated early in the disease course, can effectively improve the prognosis of these patients. ${ }^{14-20}$ However, these agents have also been associated with increased risk of developing serious infections in some studies, but not others. ${ }^{21-24}$ Because serious infection is a relatively uncommon adverse event (AE), an abundance of published safety data come from analyses of administrative claims from large populations. ${ }^{23-28}$

The present study focused on determining whether the BBCIC DRN (a) provides sufficient sample size and exposed follow-up time and (b) contains key data elements needed to ascertain exposure to biologics, define disease-based cohorts, and identify study outcomes.

\section{Methods}

\section{Study Design and Population}

We conducted a retrospective cohort study using curated data from the BBCIC DRN held in the Sentinel common data model format. The study population included individuals enrolled in one of the participating plans during the study period between January 1, 2006, and September 30, 2015. We identified all new users of a conventional or biologic disease-modifying antiinflammatory agent (Appendix A, available in online article) during the study period. New user patient episodes were defined as patients who initiated such an agent without any previous use in the prior 365 days. The date of treatment initiation was defined as the index date, and the 365 days preceding the index date was defined as the baseline period. In addition, eligible patient episodes were required to satisfy the following inclusion criteria: (a) a diagnosis of rheumatoid arthritis (RA), psoriasis, psoriatic arthritis, ankylosing spondylitis, ulcerative colitis, or Crohn disease identified from physician encounters any time prior to treatment initiation; (b) at least 18 years of age at the time of treatment initiation; and (c) at least 12 months of continuous medical and pharmacy coverage prior to treatment initiation. Follow-up started on the index date and ended at the earliest of the following: occurrence of outcome (e.g., hospitalized infection, described in detail below), treatment discontinuation or switching (described in detail below), disenrollment (which may indicate health plan change or death), or end of the study period.

Three cohorts of patient episodes were assembled based on disease diagnosis, which included: (a) RA; (b) psoriasis, psoriatic arthritis, and ankylosing spondylitis; and (c) ulcerative colitis and Crohn disease, collectively referred to as inflammatory bowel disease (IBD). All analyses were conducted independently within each cohort. A patient diagnosed with multiple autoimmune conditions may be included in more than one cohort. Additionally, prior use of methotrexate in the baseline period was required for patients in the RA cohort to create a more homogeneous cohort of patients who failed their initial methotrexate therapy. Patients were excluded from the analyses if they (a) ever had nonmelanoma skin cancer based on all available data, (b) had an immune-compromising condition (organ transplantation, HIV, advanced kidney/liver disease) based on all available data, or (c) had a serious infection any time during the 183-day period before the index date. Patients were censored if they developed an immune-compromising condition during follow-up.

\section{Medication Exposure and Covariates}

The medications of interest included anti-TNF biologics (adalimumab, certolizumab, etanercept, golimumab, infliximab), non-TNF biologics (abatacept, natalizumab, rituximab, tocilizumab, ustekinumab, vedolizumab), the oral Janus kinase inhibitor (tofacitinib), and nonbiologic disease-modifying agents (azathioprine, hydroxychloroquine, leflunomide, methotrexate, sulfasalazine, and 6-mercaptopurine). Biologics (e.g., secukinumab) approved close to the end of the study period (September 30, 2015) were not included, as it was unlikely that there would be sufficient numbers of new users to conduct the intended analysis. Appendix A presents medications examined in each of the 3 cohorts, as not all the medications are approved for all the conditions.

Exposure to the medications was ascertained based on Healthcare Common Procedure Coding System (HCPCS) codes for infused medications and National Drug Code (NDC) numbers for pharmacy-dispensed medications. Because HCPCS codes do not have a built-in days supply, the dosing interval 
FIGURE 1 Study Flow for Each Drug Cohort

\section{A. RA CONSORT Diagram}

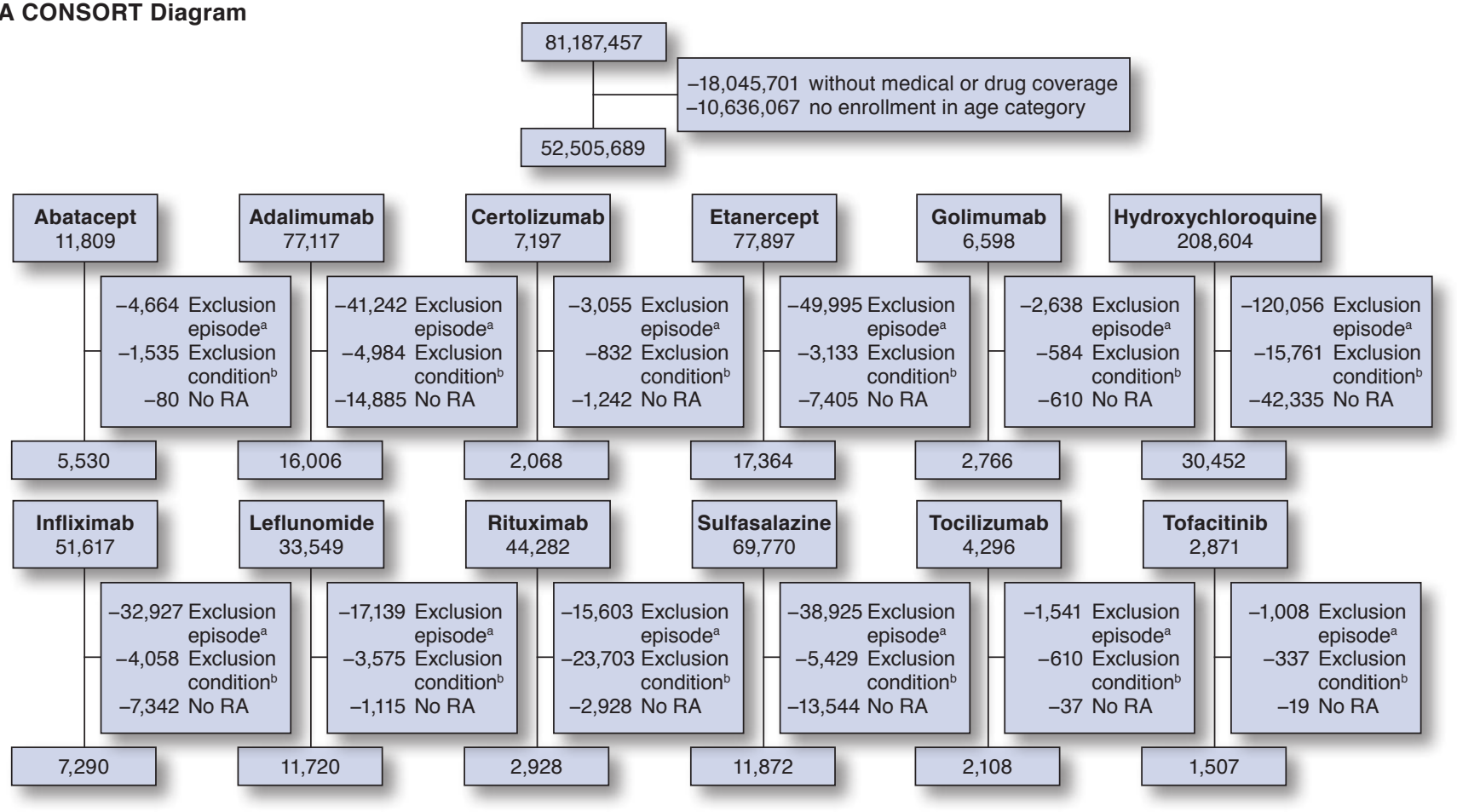

\section{B. PsO, PsA, and AS CONSORT Diagram}

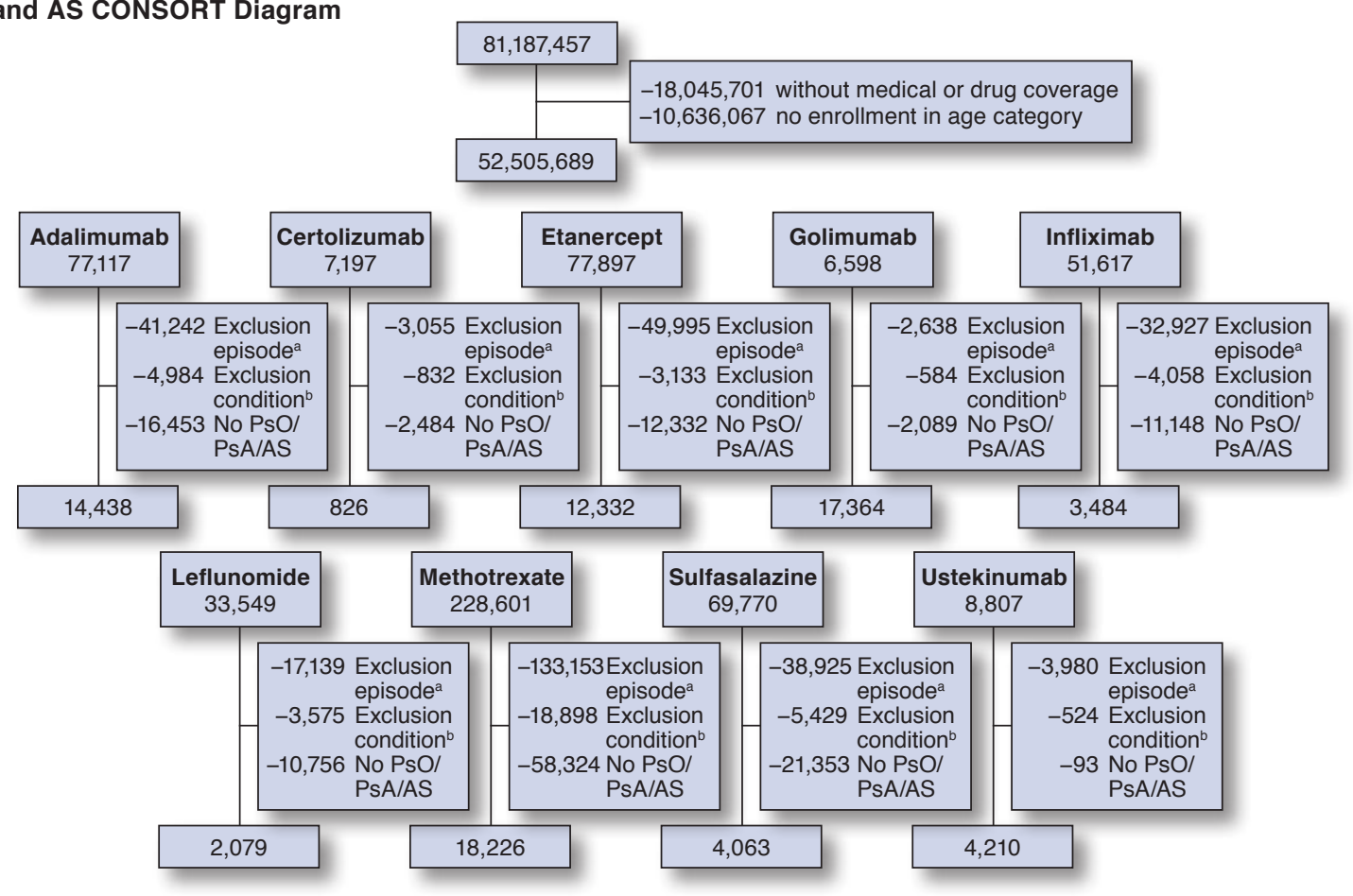




\section{IBD CONSORT Diagram}

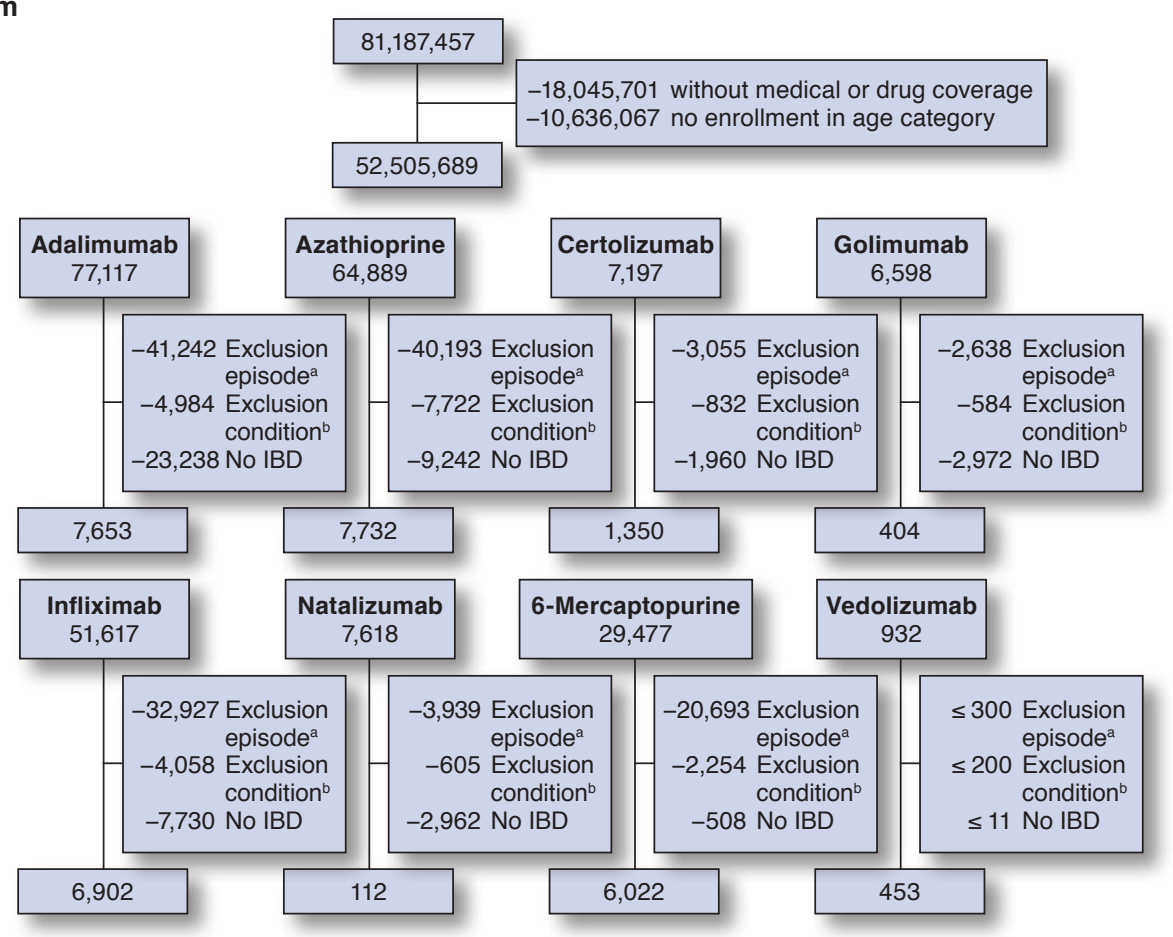

axclusion episodes based on claim in query period, episode incident, and pre-index enrollment criterion.

${ }^{b}$ Exclusions based on nonmelanoma cancer, immunocompromising conditions, and serious infection code lists.

AS = ankylosing spondylitis; CONSORT = Consolidated Standards of Reporting Trials; IBD=inflammatory bowel disease; PsA = psoriatic arthritis; PsO=psoriasis; $R A=$ rheumatoid arthritis.

provided on the FDA-approved label was applied to impute the days supply and determine exposure status during follow-up. For example, patient episodes were considered to be exposed for 56 days after receiving an infliximab infusion.

Because a biologic may be used as monotherapy or in combination with 1 or more nonbiologic disease-modifying medications, patient episodes were classified into 1 of 3 mutually exclusive categories on the index date: (a) initiated any anti-TNF biologic (regardless of nonbiologic use); (b) initiated any non-TNF antagonist biologics (regardless of nonbiologic use, RA only [Appendix A]); and (c) initiated any nonbiologic disease-modifying agents (no biologic use). Only the first patient episode was used in the outcome ascertainment analyses. During follow-up, treatment episodes were truncated when a patient episode switched within (from one anti-TNF biologic to another) or between the categories (step up from nonbiologic to biologic).

Age, sex, and calendar year of treatment initiation were ascertained on the index date. Other covariates ascertained during the 183-day period before the index date included oral glucocorticoid use, comorbidities (Charlson Comorbidity Index and individual components of the index), and health services use (e.g., numbers of inpatient visits, distinct classes of medications prescribed).

\section{Outcomes}

The study included 2 safety outcomes: serious infection and pneumonia. Serious infection was defined as incident cases of infection that required an emergency department (ED) visit or hospitalization. Cases were identified based on the presence of a discharge diagnosis code for infection of the respiratory tract, skin and soft tissue, genitourinary tract, gastrointestinal tract, or central nervous system or septicemia/sepsis. Pneumonia was defined as incident cases of pneumonia that required an ED visit or hospitalization and were identified based on a discharge diagnosis code for pneumonia from an ED visit or hospitalization.

\section{Statistical Analysis}

This study leveraged the FDA Sentinel System infrastructure, including the common data model, Sentinel-curated data (common data model v6.0.2), and modular programs for data 
TABLE 1 Baseline Patient Characteristics, by Disease Cohort and by Therapy Class ${ }^{a}$

\begin{tabular}{|c|c|c|c|c|c|c|}
\hline All Therapies & \multicolumn{2}{|c|}{ Anti-TNF Biologic } & \multicolumn{2}{|c|}{ Non-TNF Biologic } & \multicolumn{2}{|c|}{ Nonbiologic } \\
\hline \multicolumn{7}{|l|}{ Rheumatoid arthritis $(n=115,118)$} \\
\hline Total treated $\mathrm{N}=115,118$ & \multicolumn{2}{|c|}{$\mathrm{n}=35,926$} & \multicolumn{2}{|c|}{$\mathrm{n}=25,737$} & \multicolumn{2}{|c|}{$\mathrm{n}=53,455$} \\
\hline Age, mean, SD & 51 & 13.1 & 60.7 & 13.8 & 54 & 13.6 \\
\hline \multicolumn{7}{|l|}{ Age groups, years, n (\%) } \\
\hline $18-49$ & 15,671 & $(43.6)$ & 5,384 & $(20.9)$ & 19,458 & $(36.4)$ \\
\hline $50-64$ & 15,986 & $(44.5)$ & 10,883 & $(42.3)$ & 23,760 & $(44.4)$ \\
\hline $65-79$ & 3,757 & $(10.5)$ & 7,157 & $(27.8)$ & 8,462 & (15.8) \\
\hline $80+$ & 512 & (1.4) & 2,313 & $(9.0)$ & 1,775 & (3.3) \\
\hline \multicolumn{7}{|l|}{ Gender, n (\%) } \\
\hline Female & 24,353 & $(67.8)$ & 14,940 & $(58.0)$ & 40,681 & $(76.1)$ \\
\hline Male & 11,573 & $(32.2)$ & 10,797 & $(42.0)$ & 12,774 & $(23.9)$ \\
\hline CCI, mean, SD & 0.3 & (1.0) & 1.5 & $(2.0)$ & 0.4 & $(1.2)$ \\
\hline \multicolumn{7}{|l|}{ Health services utilization, mean, SD } \\
\hline Ambulatory encounters & 10.6 & $(8.7)$ & 14.6 & $(10.9)$ & 10.8 & $(9.4)$ \\
\hline ED encounters & 0.1 & $(0.4)$ & 0.1 & $(0.5)$ & 0.1 & $(0.5)$ \\
\hline Inpatient hospital encounters & 0.0 & $(0.2)$ & 0.1 & $(0.5)$ & 0.0 & $(0.2)$ \\
\hline Nonacute institutional encounters & 0.0 & $(0.1)$ & 0.0 & $(0.1)$ & 0.0 & $(0.1)$ \\
\hline Other ambulatory encounters & 2.9 & $(4.6)$ & 3.6 & $(5.5)$ & 2.9 & $(5.0)$ \\
\hline Filled prescriptions & 21.1 & $(16.1)$ & 18.7 & $(16.5)$ & 18.3 & $(15.6)$ \\
\hline Unique medical products & 7.9 & $(5.0)$ & 7.7 & $(5.3)$ & 7.3 & $(4.9)$ \\
\hline Unique drug classes & 7.5 & $(4.6)$ & 7.4 & $(5.0)$ & 7.0 & $(4.6)$ \\
\hline \multicolumn{7}{|c|}{ Psoriasis, psoriatic arthritis, ankylosing spondylitis $(n=60,700)$} \\
\hline Total treated $\mathrm{N}=60,700$ & \multicolumn{2}{|c|}{ Total $\mathrm{n}=26,812$} & & & \multicolumn{2}{|c|}{ Total $\mathrm{n}=33,888$} \\
\hline Age, mean, SD & 48.0 & $(13.4)$ & & & 53.6 & $(14.3)$ \\
\hline \multicolumn{7}{|l|}{ Age groups, years, $\mathrm{n}(\%)$} \\
\hline $18-49$ & 14,347 & $(53.5)$ & & & 13,140 & $(38.8)$ \\
\hline $50-64$ & 10,102 & $(37.7)$ & & & 13,956 & $(41.2)$ \\
\hline $65-79$ & 2,103 & $(7.8)$ & & & 5,520 & $(16.3)$ \\
\hline $80+$ & 260 & $(1.0)$ & & & 1,272 & (3.8) \\
\hline \multicolumn{7}{|l|}{ Gender, n (\%) } \\
\hline Female & 13,429 & $(50.1)$ & & & 19,851 & $(58.6)$ \\
\hline Male & 13,383 & $(49.9)$ & & & 14,037 & $(41.4)$ \\
\hline CCI, mean, SD & 0.3 & $(1.0)$ & & & 0.7 & $(1.8)$ \\
\hline \multicolumn{7}{|l|}{ Health services utilization, mean, SD } \\
\hline Ambulatory encounters & 9.9 & $(9.2)$ & & & 11.5 & $(11.1)$ \\
\hline ED encounters & 0.1 & $(0.4)$ & & & 0.1 & $(0.5)$ \\
\hline Inpatient hospital encounters & 0.0 & $(0.2)$ & & & 0.1 & $(0.4)$ \\
\hline Nonacute institutional encounters & 0.0 & $(0.1)$ & & & 0.0 & $(0.1)$ \\
\hline Other ambulatory encounters & 2.5 & $(4.7)$ & & & 3.0 & $(5.6)$ \\
\hline Filled prescriptions & 16.9 & $(15.3)$ & & & 17.3 & $(15.5)$ \\
\hline Unique medical products & 6.8 & $(4.9)$ & & & 7.2 & $(5.0)$ \\
\hline Unique drug classes & 6.3 & $(4.6)$ & & & 6.8 & $(4.7)$ \\
\hline
\end{tabular}

management and analysis. ${ }^{13}$ Specifically, cohort identification and descriptive analysis (Cohort ID and Descriptive Analysis v5.0.3) Level-1 queries (created using SAS version 9.4 [SAS Institute, Cary, NC]) were executed to identify the cohorts, ascertain exposures and outcomes, and produce unadjusted incidence rates. ${ }^{29}$ Descriptive statistics (mean [standard deviation], number [percentage]) of patient characteristics ascertained during baseline or on the index date were calculated (before implementation of the exclusion due to nonmelanoma cancer). Crude incidence rates and 95\% confidence intervals of serious infection and pneumonia during follow-up were calculated, stratified by categories of medications initiated (defined above: nonbiologic disease-modifying agents, anti-TNF biologics, and non-TNF biologics). Crude incidence rates are reported as observed, without adjustment for potential confounding variables such as oral glucocorticoid use. Data analyses were performed separately for each of the 3 cohorts. Codes used to identify exposure and outcome and to 
TABLE 1 Baseline Patient Characteristics, by Disease Cohort and by Therapy Class $^{\mathrm{a}}$ (continued)

\begin{tabular}{|c|c|c|c|c|c|}
\hline All Therapies & \multicolumn{2}{|c|}{ Anti-TNF Biologic } & Non-TNF Biologic & \multicolumn{2}{|c|}{ Nonbiologic } \\
\hline \multicolumn{6}{|c|}{ Inflammatory bowel disease $(n=32,938)$} \\
\hline Total treated $\mathrm{N}=32,938$ & \multicolumn{2}{|c|}{ Total $n=16,503$} & & \multicolumn{2}{|c|}{ Total $n=16,435$} \\
\hline Age, mean, SD & 44.6 & $(15.5)$ & & 44.7 & (15.4) \\
\hline \multicolumn{6}{|l|}{ Age groups, years, n (\%) } \\
\hline $18-49$ & 10,154 & $(61.5)$ & & 10,143 & $(61.7)$ \\
\hline $50-64$ & 4,789 & $(29.0)$ & & 4,718 & $(28.7)$ \\
\hline $65-79$ & 1,362 & $(8.3)$ & & 1,370 & (8.3) \\
\hline $80+$ & 198 & $(1.2)$ & & 204 & $(1.2)$ \\
\hline \multicolumn{6}{|l|}{ Gender, n (\%) } \\
\hline Female & 10,154 & $(61.5)$ & & 10,143 & $(61.7)$ \\
\hline Male & 4,789 & $(29.0)$ & & 4,718 & $(28.7)$ \\
\hline CCI, mean, SD & 0.6 & $(1.2)$ & & 0.6 & $(1.4)$ \\
\hline \multicolumn{6}{|l|}{ Health services utilization, mean, SD } \\
\hline Ambulatory encounters & 10.7 & $(8.6)$ & & 14.2 & $(9.9)$ \\
\hline ED encounters & 0.2 & $(0.6)$ & & 0.2 & $(0.7)$ \\
\hline Inpatient hospital encounters & 0.2 & $(0.5)$ & & 0.1 & $(0.4)$ \\
\hline Nonacute institutional encounters & 0 & $(0)$ & & 0 & $(0.1)$ \\
\hline Other ambulatory encounters & 3.1 & $(4.9)$ & & 4 & $(6.8)$ \\
\hline Filled prescriptions & 17.5 & $(15)$ & & 19.7 & $(16.1)$ \\
\hline Unique medical products & 7.1 & (4.9) & & 7.7 & (5) \\
\hline Unique drug classes & 6.9 & $(4.6)$ & & 7.6 & $(4.8)$ \\
\hline
\end{tabular}

implement inclusion and exclusion criteria are available in Appendix B (available in online article).

\section{Results}

Figure 1 shows a study flow for each drug cohort of interest within each clinical condition of interest. We identified 3 large cohorts of patient drug episodes, including 111,611 patient episodes with RA (75\% female; Figure 1A); 61,050 with psoriasis, psoriatic arthritis, ankylosing spondylitis (51\% female; Figure 1B); and 30,628 with IBD (52\% female; Figure 1C). Across all 3 cohorts, patients between 18 and 64 years of age accounted for more than $85 \%$ of the total population. Approximately half of the patients initiated a biologic (50\% in RA; $60 \%$ in psoriasis, psoriatic arthritis, ankylosing spondylitis; and 55\% in IBD patients). Among RA patients, we identified a total of 1,512 tofacitinib new patient episodes. Because we expected very low numbers of patients with either study outcome, tofacitinib new users were not included in the analyses to assess crude incidence rates. Details of patient baseline characteristics are presented in Table 1.

Patients in the 3 cohorts (RA; psoriasis, psoriatic arthritis, ankylosing spondylitis; IBD) contributed a total of 47,580 , 22,076, and 15,251 person-years (PYs) of exposed follow-up time, respectively, to the analysis of serious infection. The overall estimated incidence rate of serious infection was 9.8 (95\% confidence interval $[\mathrm{CI}]=9.5-10.0)$ cases per $100 \mathrm{PYs}$ in
RA; $7.1(\mathrm{CI}=6.8-7.5)$ in psoriasis, psoriatic arthritis, and ankylosing spondylitis; and $14.2(\mathrm{CI}=13.6-14.8)$ in IBD patients. When stratified by age, numerically higher incidence rates were observed in older age groups, and a gradient was observed across 3 cohorts and across all treatment categories (Table 2).

Different from serious infection, the highest incidence rate of pneumonia was observed in RA patients ( 1.6 cases per 100 PYs; $C I=1.5-1.7$ ); followed by patients with IBD ( 1.3 cases per 100 PYs; CI=1.1-1.5); and lowest in patients with psoriasis, psoriatic arthritis, or ankylosing spondylitis (0.8 cases per 100 PYs; CI =0.7-0.9; Table 3). Again, consistently across diagnosis and treatment, numerically higher incidence rates were observed among older patients compared with younger ones.

\section{Discussion}

This study describes the utilization of biologic and nonbiologic anti-inflammatory agents and unadjusted rates of serious infection and pneumonia in 3 cohorts of patients with autoimmune diseases. This study also identifies strengths and limitations of the BBCIC DRN, along with methodologic challenges and recommendations for improving design and conduct of future comparative safety/effectiveness studies of biologics.

First, this descriptive analysis demonstrates that the BBCIC DRN provides a sufficiently large sample size and follow-up time to examine relatively uncommon AEs in patient populations and subgroups of interest. For instance, pneumonia is an 
TABLE 2 Incidence Rates of Serious Infection, Overall and Stratified

\begin{tabular}{|c|c|c|c|c|c|c|c|c|c|c|c|c|}
\hline & \multicolumn{4}{|c|}{ Rheumatoid Arthritis } & \multicolumn{4}{|c|}{$\begin{array}{l}\text { Psoriasis, Psoriatic Arthritis, } \\
\text { Ankylosing Spondylitis }\end{array}$} & \multicolumn{4}{|c|}{ Inflammatory Bowel Disease } \\
\hline & Events, $\mathbf{n}$ & PYs, $n$ & IR & $95 \% \mathrm{CI}$ & Events, $\mathbf{n}$ & PYs, $n$ & IR & $95 \% \mathrm{CI}$ & Events, $\mathbf{n}$ & PYs, $n$ & IR & $95 \%$ CI \\
\hline Overall & 4,649 & 47,580 & 9.8 & $9.5-10.0$ & 1,577 & 22,076 & 7.1 & $6.8-7.5$ & 2,169 & 15,251 & 14.2 & $13.6-14.8$ \\
\hline Anti-TNF biologics & 1,974 & 22,057 & 8.9 & & 912 & 13,423 & 6.8 & & 1,192 & 8,000 & 14.9 & \\
\hline \multicolumn{13}{|l|}{ Age, in years } \\
\hline $18-49$ & 726 & 9,730 & 7.5 & & 452 & 7,864 & 5.7 & & 807 & 5,734 & 14.1 & \\
\hline $50-64$ & 821 & 9,945 & 8.3 & & 362 & 4,868 & 7.4 & & 290 & 1,958 & 14.8 & \\
\hline $65-79$ & 355 & 2,176 & 16.3 & & 87 & 654 & 13.3 & & 81 & 287 & 28.2 & \\
\hline $80+$ & 72 & 205 & 35.1 & & 11 & 37 & 29.4 & & 14 & 21 & 67.2 & \\
\hline \multicolumn{13}{|l|}{ Gender } \\
\hline Female & 1,349 & 14,082 & 9.6 & & 460 & 5,444 & 8.4 & & 643 & 4,065 & 15.8 & \\
\hline Male & 625 & 7,975 & 7.8 & & 452 & 7,979 & 5.7 & & 549 & 3,935 & 14.0 & \\
\hline Non-TNF biologics & 648 & 5,137 & 12.6 & & & & & & & & & \\
\hline \multicolumn{13}{|l|}{ Age, in years } \\
\hline $18-49$ & 146 & 1,608 & 9.1 & & & & & & & & & \\
\hline $50-64$ & 248 & 2,323 & 10.7 & & & & & & & & & \\
\hline $65-79$ & 204 & 1,048 & 19.5 & & & & & & & & & \\
\hline $80+$ & 50 & 158 & 31.7 & & & & & & & & & \\
\hline \multicolumn{13}{|l|}{ Gender } \\
\hline Female & 507 & 4,095 & 12.4 & & & & & & & & & \\
\hline Male & 141 & 1,041 & 13.5 & & & & & & & & & \\
\hline Nonbiologics & 2,027 & 20,386 & 9.9 & & 665 & 8,652 & 7.7 & & 977 & 7,251 & 13.5 & \\
\hline \multicolumn{13}{|l|}{ Age, in years } \\
\hline $18-49$ & 555 & 7,310 & 7.6 & & 205 & 3,799 & 5.4 & & 577 & 4,793 & 12.0 & \\
\hline $50-64$ & 799 & 9,441 & 8.5 & & 288 & 3,702 & 7.8 & & 296 & 2,007 & 14.8 & \\
\hline $65-79$ & 530 & 3,094 & 17.1 & & 139 & 1,027 & 13.5 & & 86 & 420 & 20.5 & \\
\hline $80+$ & 143 & 542 & 26.4 & & 33 & 124 & 26.6 & & 18 & 30 & 59.9 & \\
\hline \multicolumn{13}{|l|}{ Gender } \\
\hline Female & 1,512 & 15,643 & 9.7 & & 413 & 4,463 & 9.3 & & 484 & 3,421 & 14.1 & \\
\hline Male & 515 & 4743 & 10.9 & & 252 & 4,189 & 6.0 & & 493 & 3,829 & 12.9 & \\
\hline
\end{tabular}

infrequent $\mathrm{AE}$, which occurred at crude incidence rates of 0.8 1.6 cases per 100 PYs across the 3 cohorts in this study. Still, the large size of the BBCIC DRN enabled the identification of $50,106,22,819$, and 16,377 PYs of exposed follow-up time and 792,187 , and 214 incident cases of pneumonia among patients with RA; psoriasis, psoriatic arthritis, or ankylosing spondylitis; and IBD, respectively. The BBCIC DRN also contains adequately detailed data elements to characterize the study populations that included comorbidities and use of prescription medications and other health services. These measures have the potential to serve as proxies of RA severity, health status, and health-seeking behavior that may be included as potential confounders to be adjusted for in future studies.

The crude incidence rates of serious infection among patients in the RA and IBD cohorts initiating an anti-TNF agent in the present study were numerically higher (8.9 and 14.9 per 100 PYs, respectively) than the crude rates reported in a similar study by Grijalva et al. (2011; 8.2 and 10.9 per 100 PYs, respectively). ${ }^{23}$ This is likely due to differences in how outcomes are defined: the present study identified serious infections from both ED visits and hospitalizations and included both principal and secondary diagnoses, whereas the Grijalva study only included the principal diagnosis from hospitalizations. ${ }^{23}$ Likewise, the unadjusted crude incidence rate of serious infection among RA patients aged 65 years or older in the present study was 18.7 per 100 PYs (1,354 cases; $7,223 \mathrm{PYs}$ ), which is qualitatively higher than the risk estimates reported among elderly RA patients (e.g., 13.1-18.7 per 100 PYs) with Medicare coverage. ${ }^{21}$

Another key strength of the BBCIC DRN is the ability to leverage the Sentinel infrastructure for data management and analysis, including the common data model and analytic tools, which were developed to support active postmarketing drug safety surveillance. ${ }^{13}$ This approach offered significant computational efficiency to conduct multidatabase studies, as it allowed rapid local execution of centrally developed modular programs concurrently by multiple data partners using the curated data in the common data model format. ${ }^{30}$ This study 


\begin{tabular}{|c|c|c|c|c|c|c|c|c|c|c|c|c|}
\hline & \multicolumn{4}{|c|}{ Rheumatoid Arthritis } & \multicolumn{4}{|c|}{$\begin{array}{l}\text { Psoriasis, Psoriatic Arthritis, } \\
\text { Ankylosing Spondylitis }\end{array}$} & \multicolumn{4}{|c|}{ Inflammatory Bowel Disease } \\
\hline & Events, $\mathbf{n}$ & PYs, n & IR & $95 \%$ CI & Events, $\mathbf{n}$ & PYs, $n$ & IR & $95 \%$ CI & Events, $\mathbf{n}$ & PYs, $n$ & IR & $95 \% \mathrm{CI}$ \\
\hline Overall & 792 & 50,106 & 1.6 & $1.5-1.7$ & 187 & 22,829 & 0.8 & $0.7-0.9$ & 214 & 16,377 & 1.3 & $1.1-1.5$ \\
\hline Anti-TNF biologics & 342 & 23,196 & 1.5 & & 98 & 13,939 & 0.7 & & 114 & 8,648 & 1.3 & \\
\hline \multicolumn{13}{|l|}{ Age, in years } \\
\hline $18-49$ & 71 & 10,156 & 0.7 & & 36 & 8,114 & 0.4 & & 57 & 6,149 & 0.9 & \\
\hline $50-64$ & 160 & 10,406 & 1.5 & & 49 & 5,080 & 1.0 & & 42 & 2,135 & 2.0 & \\
\hline $65-79$ & 84 & 2,411 & 3.5 & & 12 & 704 & 1.7 & & 14 & 333 & 4.2 & \\
\hline $80+$ & 27 & 224 & 12.1 & & $<10$ & 41 & & & $<10$ & 32 & & \\
\hline \multicolumn{13}{|l|}{ Gender } \\
\hline Female & 226 & 14,850 & 1.5 & & 44 & 5,689 & 0.8 & & 62 & 4413 & 1.4 & \\
\hline Male & 116 & 8,345 & 1.4 & & 54 & 8,251 & 0.7 & & 52 & 4235 & 1.2 & \\
\hline Non-TNF biologics & 111 & 5,464 & 2.0 & & & & & & & & & \\
\hline \multicolumn{13}{|l|}{ Age, in years } \\
\hline $18-49$ & 20 & 1,661 & 1.2 & & & & & & & & & \\
\hline $50-64$ & 43 & 2,453 & 1.8 & & & & & & & & & \\
\hline $65-79$ & 33 & 1,163 & 2.8 & & & & & & & & & \\
\hline $80+$ & 15 & 187 & 8.0 & & & & & & & & & \\
\hline \multicolumn{13}{|l|}{ Gender } \\
\hline Female & 82 & 4,348 & 1.9 & & & & & & & & & \\
\hline Male & 29 & 1,115 & 2.6 & & & & & & & & & \\
\hline Nonbiologics & 339 & 21,446 & 1.6 & & 89 & 8,890 & 1.0 & & 100 & 7,728 & 1.3 & \\
\hline \multicolumn{13}{|l|}{ Age, in years } \\
\hline $18-49$ & 48 & 7,571 & 0.6 & & 21 & 3,863 & 0.5 & & 47 & 5,045 & 0.9 & \\
\hline $50-64$ & 127 & 9,862 & 1.3 & & 36 & 3,804 & 0.9 & & 36 & 2,161 & 1.7 & \\
\hline $65-79$ & 121 & 3,406 & 3.6 & & 26 & 1,087 & 2.4 & & 13 & 486 & 2.7 & \\
\hline $80+$ & 43 & 608 & 7.1 & & $<10$ & 137 & & & $<10$ & 37 & & \\
\hline \multicolumn{13}{|l|}{ Gender } \\
\hline Female & 244 & 16,427 & 1.5 & & 52 & 4,609 & 1.1 & & 39 & 3,666 & 1.1 & \\
\hline Male & 95 & 5,019 & 1.9 & & 37 & 4,281 & 0.9 & & 61 & 4,062 & 1.5 & \\
\hline
\end{tabular}

also leveraged the Sentinel Operation Center query-specifications document to communicate study design and operational details in a standardized format that enables reliable replication. Thus, it is critical that the specifications are developed thoughtfully and appropriately tailored for the specific needs of each project.

Because of the complexity and importance of these tools, we recommend that research teams be trained on the Sentinel infrastructure to obtain an in-depth understanding of the common data model structure, the capability of the analytic tools, and the specifications document. As previously reported, we recommend a phased approach in which results from a test database or one data partner are reviewed by the investigators before executing the analytic programs across all data partners. ${ }^{31}$ This approach allows the investigators to revise the study design, if necessary, and thus avoid executing analytic programs multiple times across all data partners, which requires significant effort and resources. For example, our research team worked closely to determine the allowable gap to bridge self-injectable biologics given that HCPCS codes in a medical procedure table do not contain an adjudicated days supply field found in pharmacy-dispensing tables. Finally, an analyst with detailed knowledge of the Sentinel data model and analytic tools is an invaluable resource who can also offer insights from experience that further ensure the successful implementation of a study.

\section{Limitations}

We identified study limitations in our ability to conduct biologic and biosimilar surveillance. In the present study, new users are defined as those new to the entire class of study medications (e.g., anti-TNF biologic) and not just the specific cohort-defining medication (e.g., etanercept). As this was a feasibility assessment of the BBCIC DRN to address biologic and biosimilar surveillance, future work will need to be conducted at the medication and manufacturer level. The research team made the decision to avoid selection bias related to medication effects common to the class. For instance, a patient may be 
selected to initiate rituximab versus another anti-TNF biologic if the patient experienced a severe injection site reaction to a previous anti-TNF biologic.

Given that biosimilar initiators are likely to include patients who have switched from the reference biologic, how to handle switching and compare switchers to those who opt to remain on the reference biologic is of critical importance to the biosimilar research study design. ${ }^{32}$ For example, patients who switched to a biosimilar from a reference biologic to comply with a formulary decision may be different from patients who switched from a structurally unrelated biologic with regard to their baseline risk of developing an AE. These 2 scenarios of switching to a biosimilar may yield very different safety results, and it would be useful if these different scenarios are captured.

Another methodologic challenge that affects all biologics (including biosimilars) is that a specific HCPCS code that uniquely identifies the medication is usually not assigned until the beginning of the following calendar year after FDA approval. Our study did not utilize these nonspecific codes. Additionally, unique to biosimilars, since 2016, the Centers for Medicare and Medicaid Services (CMS) assigned the same HCPCS code to biosimilars of the same reference biologic and used modifiers to identify the manufacturers of the biosimilars. While the policy was reversed in November 2017 and CMS now assigns an individual HCPCS code to each biosimilar, ${ }^{33}$ there is a need to empirically assess the utilization of the modifiers for biosimilars that are affected.

To address the issues identified early in this study, BBCIC commissioned 2 additional work groups to address the challenges of drug switching and exposure identification. First, specific methodological recommendations are needed to handle the complexity of switching in the context of comparative effectiveness or safety studies that may involve several classes of biologics with potentially multiple biosimilars for each reference biologic. Second, an empirical examination of the BBCIC DRN databases has been conducted to assess the utilization of HCPCS modifiers and NDC numbers in medical claims. ${ }^{34}$ The goal was to determine the potential of using these data fields to improve the identification of biologic and biosimilar exposure that is billed using nonspecific HCPCS codes, biosimilars that do not have a modifier to specify the manufacturer under the older CMS policy, and administrations that occurred at inpatient and ED settings that would have otherwise not been captured. While the incorporation of HCPCS modifiers and medical claim NDC numbers may complicate exposure ascertainment, it has the potential to meaningfully improve the capture of biologics and biosimilars. While the FDA has issued guidance on nonproprietary naming of biologicals products, it will take time for widespread adoption into our electronic medical records and routine utilization in administrative claims processing. ${ }^{35}$ The 2 BBCIC work groups are currently ongoing, and the findings will inform future biosimilar surveillance activities.

Finally, as the existing BBCIC DRN is based on administrative claims data, there are challenges with identifying outcome measures related to clinical effectiveness. This study helped confirm whether effectiveness could be quantified either directly or indirectly (e.g., surrogate measures) and provided a means of identifying opportunities where data enrichment of the existing DRN will be necessary to better answer some effectiveness outcomes of interest. Work is ongoing to develop more robust capabilities to support BBCIC research, and this study provided a necessary baseline from which to build.

\section{Conclusions}

This descriptive study demonstrates that the BBCIC DRN is a useful source for biologic surveillance. Efforts are currently in progress to address the methodological challenges identified in the study, such as the ascertainment of biosimilars in the DRN. The lessons learned are translatable to other future studies, including the importance of a thorough appraisal of methods used to assess and classify exposure, an in-depth understanding of the Sentinel distributed system and tools, and a phased analytic approach.

\section{Authors}

JIE ZHANG, PhD; GAYATHRI SRIDHAR, PhD; and KEVIN HAYNES, PharmD, MSCE, HealthCore, Wilmington, Delaware. CHARLES E. BARR, MD, MPH, and BERNADETTE EICHELBERGER, PharmD, AMCP, Alexandria, Virginia. CATHERINE M. LOCKHART, PharmD, PhD, Biologics and Biosimilars Collective Intelligence Consortium, Alexandria, Virginia. JAMES MARSHALL, MPH, and JEFFREY S. BROWN, PhD, Harvard Pilgrim Health Care Institute, Boston, Massachusetts. JERRY CLEWELL, PharmD, MBA, and CYNTHIA HOLMES, PharmD, MS, AbbVie, North Chicago, Illinois. NEIL A. ACCORTT, PhD, Amgen, Thousand Oaks, California; JEFFREY R. CURTIS, MD, MS, MPH, University of Alabama at Birmingham; and CHERYL N. MCMAHILL-WALRAVEN, PhD, Healthagen, a CVS Health company, Blue Bell, Pennsylvania.

AUTHOR CORRESPONDENCE: Kevin Haynes, PharmD, MSCE, HealthCore, 123 Justison St., Ste. 200, Wilmington, DE 19801. Tel.: 302.230.2108; E-mail: khaynes@healthcore.com.

\section{DISCLOSURES}

This study was funded by the Biologics and Biosimilars Collective Intelligence Consortium (BBCIC). HealthCore conducted this study in collaboration with Harvard Pilgrim Health Care. Zhang and Sridhar were employed by HealthCore at the time of this study. Haynes is employed by HealthCore funded by PCORI, the NIH, and the FDA. Barr and Eichelberger were employed by AMCP at the time of this study. Lockhart is employed by the BBCIC. Holmes and Clewell are employed by AbbVie. Accrott is an employee of and shareholder in Amgen. Marshall and Brown are employed by Harvard Pilgrim Health Care. Barr is a shareholder in Roche/Genentech. Curtis has received 
research grants from and consults with the following: Amgen, AbbVie, BMS, CORRONA, Lilly, Janssen, Myriad, Pfizer, Roche, Regeneron, and UCB. Brown has received research grants from GSK and Pfizer and consulting fees from Bayer, Roche, and Jazz Pharmaceuticals, along with funding from the Reagan-Udall Foundation for the FDA to conduct studies for medical product manufacturers, including Eli Lilly, Novartis, Abbvie, and Merck. Brown is also funded by PCORI, the NIH, and the FDA. McMahill-Walraven subcontracts with Harvard Pilgrim Health Care Institute for public health and safety surveillance distributed data network activtities and with the FDA, GSK, and Pfizer. She also reports fees from Reagan Udall Foundation for the FDA and the Patient Centered Outcomes Research Institute.

\section{ACKNOWLEDGMENTS}

The authors thank the following members of the BBCIC Anti-inflammatory Work Group for their contributions: Sanjeev Balu, Karen Birmingham (GHC), Brian Bradbury (Amgen), Yanni Fan, Timothy Juday (Boehringer Ingelheim), Erin M. Lopata (University of Pittsburgh Medical Center Health Plan), Michael Nayden (Aetna), Pamela Pawloski (Health Partners), and William Yoon (Sandoz). The authors also acknowledge Cara McDermott (BBCIC) and Aaron Mendelsohn (Harvard Pilgrim Health Care Institute) for their critical review of this work. Their comments greatly improved this manuscript.

\section{REFERENCES}

1. Holzmann J, Balser S, Windisch J. Totality of the evidence at work: the first U.S. biosimilar. Expert Opin Biol Ther. 2016;16(2):137-42.

2. U.S. Food and Drug Administration. Biosimilar product information. 2018. Available at: https://www.fda.gov/Drugs/DevelopmentApproval Process/HowDrugsareDevelopedandApproved/ApprovalApplications/ TherapeuticBiologicApplications/Biosimilars/ucm580432.htm. Accessed March 15, 2020.

3. Zhang J, Xie F, Delzell E, et al. Trends in the use of biologic agents among rheumatoid arthritis patients enrolled in the U.S. Medicare program. Arthritis Care Res (Hoboken). 2013;65(11):1743-51.

4. McBride A, Campbell K, Bikkina M, MacDonald K, Abraham I, Balu S. Cost-efficiency analyses for the US of biosimilar filgrastim-sndz, reference filgrastim, pegfilgrastim, and pegfilgrastim with on-body injector in the prophylaxis of chemotherapy-induced (febrile) neutropenia. J Med Econ. 2017;20(10):1083-93

5. Mulcahy AW, Hlavka JP, Case SR. Biosimilar cost savings in the United States: initial experience and future potential. Rand Health Q. 2018;7(4):3.

6. U.S. Food and Drug Administration. Biosimilar and interchangeable products. Available at: https://www.fda.gov/Drugs/DevelopmentApprovalProcess/ HowDrugsareDevelopedandApproved/ApprovalApplications/Therapeutic BiologicApplications/Biosimilars/ucm580419.htm. Accessed March 15, 2020.

7. U.S. Food and Drug Administration. Scientific considerations in demonstrating biosimilarity to a reference product: guidance for industry. April 2015. Available at: https://www.fda.gov/media/82647/download. Accessed March 20, 2020.

8. Kobayashi T, Kamada I, Komura J, Toyoshima S, Ishii-Watabe A. Comparative study of the number of report and time-to-onset of the reported adverse event between the biosimilars and the originator of filgrastim. Pharmacoepidemiol Drug Saf. 2017;26(8):917-24

9. Douglas AG, Schwab P, Lane D, Kennedy K, Slabaugh SL, Bowe A. A comparison of brand and biosimilar granulocyte-colony stimulating factors for prophylaxis of chemotherapy-induced febrile neutropenia. J Manag Care Spec Pharm. 2017;23(12):1221-26. Available at: https://www.jmcp.org/doi/ full/10.18553/jmcp.2017.23.12.1221.

10. AMCP Task Force on Biosimilar Collective Intelligence Systems. Utilizing data consortia to monitor safety and effectiveness of biosimilars and their innovator products. J Manag Care Spec Pharm. 2015;21(1):23-34. Available at: https://www.jmcp.org/doi/abs/10.18553/jmcp.2015.21.1.23.
11. Mendelsohn AB, Barr CE, Brown JS, et al. Development and management of a distributed research network for evaluating real-would outcomes for originator biologics and biosimilars [abstract]. J Manag Care Spec Pharm. 2018;24(10-a Suppl):101-02. Available at: https://www.jmcp.org/doi/ pdf/10.18553/jmcp.2018.24.issue-10-a.

12. Platt R, Brown JS, Robb M, et al. The FDA Sentinel Initiative - an evolving national resource. N Engl J Med. 2018;379(22):2091-93.

13. Ball R, Robb M, Anderson SA, Dal Pan G. The FDA's Sentinel Initiativea comprehensive approach to medical product surveillance. Clin Pharmacol Ther. 2016;99(3):265-68.

14. Emery P, Breedveld FC, Hall S, et al. Comparison of methotrexate monotherapy with a combination of methotrexate and etanercept in active, early, moderate to severe rheumatoid arthritis (COMET): a randomised, doubleblind, parallel treatment trial. Lancet. 2008;372(9636):375-82.

15. Klareskog L, van der Heijde D, de Jager JP, et al. Therapeutic effect of the combination of etanercept and methotrexate compared with each treatment alone in patients with rheumatoid arthritis: double-blind randomised controlled trial. Lancet. 2004;363(9410):675-81.

16. Weinblatt ME, Keystone EC, Furst DE, et al. Adalimumab, a fully human anti-tumor necrosis factor alpha monoclonal antibody, for the treatment of rheumatoid arthritis in patients taking concomitant methotrexate: the ARMADA trial. Arthritis Rheum. 2003;48(1):35-45.

17. Genovese MC, Kremer J, Zamani O, et al. Baricitinib in patients with refractory rheumatoid arthritis. N Engl J Med. 2016;374(13):1243-52.

18. Weinblatt ME, Westhovens R, Mendelsohn AM, et al. Radiographic benefit and maintenance of clinical benefit with intravenous golimumab therapy in patients with active rheumatoid arthritis despite methotrexate therapy: results up to 1 year of the phase 3 , randomised, multicentre, double blind, placebo controlled GO-FURTHER trial. Ann Rheum Dis. 2014;73(12):2152-59.

19. Emery P, Bingham CO 3rd, Burmester GR, et al. Certolizumab pegol in combination with dose-optimised methotrexate in DMARD-naive patients with early, active rheumatoid arthritis with poor prognostic factors: 1-year results from C-EARLY, a randomised, double-blind, placebo-controlled phase III study. Ann Rheum Dis. 2017;76(1):96-104.

20. Fleischmann R, Weinblatt ME, Schiff M, et al. Patient-reported outcomes from a two-year head-to-head comparison of subcutaneous abatacept and adalimumab for rheumatoid arthritis. Arthritis Care Res (Hoboken). 2016;68(7):907-13.

21. Bongartz T, Sutton AJ, Sweeting MJ, Buchan I, Matteson EL, Montori V. Anti-TNF antibody therapy in rheumatoid arthritis and the risk of serious infections and malignancies: systematic review and meta-analysis of rare harmful effects in randomized controlled trials. JAMA. 2006;295(19):2275-85.

22. Dixon WG, Symmons DP, Lunt M, et al. Serious infection following antitumor necrosis factor alpha therapy in patients with rheumatoid arthritis: lessons from interpreting data from observational studies. Arthritis Rheum. 2007:56(9):2896-904.

23. Grijalva CG, Chen L, Delzell E, et al. Initiation of tumor necrosis factoralpha antagonists and the risk of hospitalization for infection in patients with autoimmune diseases. JAMA. 2011;306(21):2331-39.

24. Schneeweiss S, Setoguchi S, Weinblatt ME, et al. Anti-tumor necrosis factor alpha therapy and the risk of serious bacterial infections in elderly patients with rheumatoid arthritis. Arthritis Rheum. 2007;56(6):1754-64.

25. Yun H, Xie F, Delzell E, et al. Comparative risk of hospitalized infection associated with biologic agents in rheumatoid arthritis patients enrolled in Medicare. Arthritis Rheumatol. 2016;68(1):56-66.

26. Xie F, Yun H, Bernatsky S, Curtis JR. Brief report: risk of gastrointestinal perforation among rheumatoid arthritis patients receiving tofacitinib, tocilizumab, or other biologic treatments. Arthritis Rheumatol. 2016;68(11):2612-17.

27. George MD, Baker JF, Hsu JY, et al. Perioperative timing of infliximab and the risk of serious infection after elective hip and knee arthroplasty. Arthritis Care Res (Hoboken). 2017;69(12):1845-54. 
28. Yun H, Xie F, Delzell E, et al. Risks of herpes zoster in patients with rheumatoid arthritis according to biologic disease-modifying therapy. Arthritis Care Res (Hoboken). 2015;67(5):731-36.

29. The Sentinel Operations Center. Surveillance tools: routine querying tools (modular programs). Available at: https://www.sentinelinitiative.org/sentinel/ surveillance-tools/routine-querying-tools. Accessed March 15, 2020.

30. Curtis LH, Weiner MG, Boudreau DM, et al. Design considerations, architecture, and use of the Mini-Sentinel distributed data system.

Pharmacoepidemiol Drug Saf. 2012;21(Suppl 1):23-31.

31. Desai RJ, Kim SC, Curtis JR, et al. Methodologic considerations for noninterventional studies of switching from reference biologic to biosimilars. Pharmacoepidemiol Drug Saf. July 12, 2019 [Epub ahead of print]. Available at: https://onlinelibrary.wiley.com/doi/abs/10.1002/pds.4809. Accessed March 16, 2020.

32. Dutcher SK, Eworuke E, Blum MD, Ball R. Using real-world data to evaluate biosimilar switching. Pharmacoepidemiol Drug Saf. November 6, 2019 [Epub ahead of print]. Available at: https://onlinelibrary.wiley.com/doi/ abs/10.1002/pds.4899. Accessed March 16, 2020.
33. Centers for Medicare \& Medicaid Services. Final policy, payment, and quality provisions in the Medicare Physician Fee Schedule for calendar year 2019. November 1, 2018. Available at: https://www.cms.gov/newsroom/ fact-sheets/final-policy-payment-and-quality-provisions-changes-medicarephysician-fee-schedule-calendar-year. Accessed March 20, 2020

34. Zhang J, Haynes K, Mendelsohn AB, et al. Identification of exposure to reference biologics and biosimilars - use of medical claim National Drug Code and Healthcare Common Procedure Coding System Code modifier. Presented at: 35th International Conference on Pharmacoepidemiology \& Therapeutic Risk Management; August 26, 2019; Philadelphia, PA. Available at: https://www.pharmacoepi.org/pub/?id=D736A793-D93E-6FD5-A33FF2CBA3E10BA2. Accessed March 15, 2020

35. U.S. Food and Drug Administration. Nonproprietary naming of biological products: update guidance for industry. March 2019. Available at: https:// www.fda.gov/regulatory-information/search-fda-guidance-documents/nonproprietary-naming-biological-products-update-guidance-industry. Accessed March 15, 2020.

\begin{tabular}{|c|c|c|c|}
\hline \multirow[t]{2}{*}{ APPENDIX A } & \multicolumn{3}{|c|}{$\begin{array}{l}\text { Medications of Interest by Category } \\
\text { and FDA-Approved indication }\end{array}$} \\
\hline & RA & IBD & $\begin{array}{l}\text { Psoriasis, } \\
\text { Psoriatic } \\
\text { Arthritis, } \\
\text { Ankylosing } \\
\text { Spondylitis }\end{array}$ \\
\hline \multicolumn{4}{|c|}{ Anti-TNF and $\alpha 4 \beta$ l-integrin biologics } \\
\hline Adalimumaba & $\mathrm{Y}$ & $\mathrm{Y}$ & $\mathrm{Y}$ \\
\hline Certolizumab & $\mathrm{Y}$ & $\mathrm{Y}$ & $\mathrm{Y}$ \\
\hline Etanercepta & $\mathrm{Y}$ & $\mathrm{N}$ & $\mathrm{Y}$ \\
\hline Golimumab & $\mathrm{Y}$ & $\mathrm{Y}$ & $\mathrm{Y}$ \\
\hline Infliximaba & $\mathrm{Y}$ & $\mathrm{Y}$ & $\mathrm{Y}$ \\
\hline Natalizumab & $\mathrm{N}$ & $\mathrm{Y}$ & $\mathrm{N}$ \\
\hline \multicolumn{4}{|c|}{ Non-TNF- $\alpha$-antagonist biologics } \\
\hline Abatacept (T-cell target) & $\mathrm{Y}$ & $\mathrm{N}$ & $\mathrm{N}$ \\
\hline $\begin{array}{l}\text { Rituximab } \\
(\mathrm{CD} 20+\mathrm{B} \text { cells })\end{array}$ & $\mathrm{Y}$ & $\mathrm{N}$ & $\mathrm{N}$ \\
\hline $\begin{array}{l}\text { Tocilizumab (IL-6 } \\
\text { receptor target) }\end{array}$ & Y & $\mathrm{N}$ & $\mathrm{N}$ \\
\hline $\begin{array}{l}\text { Ustekinumab (IL-12 and } \\
\text { IL-23 receptor target) }\end{array}$ & $\mathrm{N}$ & $\mathrm{N}$ & $\mathrm{Ya}^{\mathrm{a}}$ \\
\hline $\begin{array}{l}\text { Vedolizumab } \\
\text { (MAdCAM-1 target) }\end{array}$ & $\mathrm{N}$ & $Y^{a}$ & $\mathrm{~N}$ \\
\hline \multicolumn{4}{|l|}{ Oral DMARD } \\
\hline $\begin{array}{l}\text { Tofacitinib } \\
\text { (JAK inhibitor) }\end{array}$ & $\mathrm{Y}^{\mathrm{a}}$ & $\mathrm{N}$ & $\mathrm{N}$ \\
\hline \multicolumn{4}{|l|}{ Nonbiologic agents } \\
\hline 6-mercaptopurine & $\mathrm{N}$ & $\mathrm{Y}$ & $\mathrm{N}$ \\
\hline Azathioprine & $\mathrm{N}$ & $\mathrm{Y}$ & $\mathrm{N}$ \\
\hline Hydroxychloroquine & $\mathrm{Y}$ & $\mathrm{N}$ & $\mathrm{N}$ \\
\hline Methotrexate & $\mathrm{N}^{\mathrm{b}}$ & $\mathrm{N}$ & $\mathrm{Y}$ \\
\hline Leflunomide & $\mathrm{Y}$ & $\mathrm{N}$ & $\mathrm{Y}$ \\
\hline Sulfasalazine & $\mathrm{Y}$ & $\mathrm{N}$ & $\mathrm{Y}$ \\
\hline \multicolumn{4}{|c|}{$\begin{array}{l}\text { aData not presented due to small numbers. } \\
\text { bPrior use of methotrexate in the previous year required of eligible } R A \text { patients. } \\
D M A R D=\text { disease-modifying antirheumatic drug; } I B D=\text { inflammatory bowel } \\
\text { disease; } R A=\text { rheumatoid arthritis; } T N F=\text { tumor necrosis factor. }\end{array}$} \\
\hline
\end{tabular}




\section{Category}

Rheumatoid arthritis (RA)

Rheumatoid arthritis (RA)

Rheumatoid arthritis (RA)

Rheumatoid arthritis (RA)

Rheumatoid arthritis (RA)

Rheumatoid arthritis (RA)

Rheumatoid arthritis (RA)

Rheumatoid arthritis (RA)

Rheumatoid arthritis (RA)

Rheumatoid arthritis (RA)

Rheumatoid arthritis (RA)

Rheumatoid arthritis (RA)

Inflammatory bowel disease (IBD)

Inflammatory bowel disease (IBD)

Inflammatory bowel disease (IBD)

Inflammatory bowel disease (IBD)

Inflammatory bowel disease (IBD)

Inflammatory bowel disease (IBD)

Inflammatory bowel disease (IBD)

Inflammatory bowel disease (IBD)

Inflammatory bowel disease (IBD)

Inflammatory bowel disease (IBD)

Inflammatory bowel disease (IBD)

Inflammatory bowel disease (IBD)

Inflammatory bowel disease (IBD)

Psoriasis (PsO)/psoriatic arthritis

(PsA)/ankylosing spondylitis (AS)

Psoriasis (PsO)/psoriatic arthritis

(PsA)/ankylosing spondylitis (AS)

Psoriasis (PsO)/psoriatic arthritis

(PsA)/ankylosing spondylitis (AS)

Psoriasis (PsO)/psoriatic arthritis

(PsA)/ankylosing spondylitis (AS)

Immunocompromising conditions

Immunocompromising conditions

Immunocompromising conditions

Immunocompromising conditions

\section{Description}

Rheumatoid arthritis (RA) Felty's syndrome

Rheumatoid arthritis (RA) Juvenile chronic polyarthritis

Rheumatoid arthritis (RA) Monoarticular juvenile rheumatoid arthritis

Rheumatoid arthritis (RA) Other

Rheumatoid arthritis (RA) Other rheumatoid arthritis with visceral or systemic involvement

Rheumatoid arthritis (RA) Other specified inflammatory

polyarthropathies

Rheumatoid arthritis (RA) Pauciarticular juvenile rheumatoid arthritis

Rheumatoid arthritis (RA) Polyarticular juvenile rheumatoid arthritis, acute

Rheumatoid arthritis (RA) Polyarticular juvenile rheumatoid arthritis, chronic or unspecified

Rheumatoid arthritis (RA) Rheumatoid arthritis

Rheumatoid arthritis (RA) Rheumatoid lung

Rheumatoid arthritis (RA) Unspecified inflammatory polyarthropathy

Inflammatory bowel disease (IBD) Left-sided ulcerative (chronic) colitis

Inflammatory bowel disease (IBD) Other ulcerative colitis

Inflammatory bowel disease (IBD) Pseudopolyposis of colon

Inflammatory bowel disease (IBD) Regional enteritis of large intestine

Inflammatory bowel disease (IBD) Regional enteritis of small intestine

Inflammatory bowel disease (IBD) Regional enteritis of small intestine with large intestine

Inflammatory bowel disease (IBD) Regional enteritis of unspecified site

Inflammatory bowel disease (IBD) Ulcerative (chronic) enterocolitis

Inflammatory bowel disease (IBD) Ulcerative (chronic) ileocolitis

Inflammatory bowel disease (IBD) Ulcerative (chronic) proctitis

Inflammatory bowel disease (IBD) Ulcerative (chronic) proctosigmoiditis

Inflammatory bowel disease (IBD) Ulcerative colitis, unspecified

Inflammatory bowel disease (IBD) Universal ulcerative (chronic) colitis

Ankylosing spondylitis (AS) Ankylosing spondylitis

Psoriasis (PsO) Other psoriasis

Psoriasis (PsO) Psoriasis related disease NEC

Psoriatic arthritis (PsA) Psoriatic arthropathy

Chronic kidney disease

Chronic kidney disease

Chronic kidney disease

Chronic kidney disease
Code

714.1

714.3

714.33

714.89

714.2

714.8

714.32

714.31

714.3

714.0

714.81

714.9

556.5

556.8

556.4

555.1

555.0

555.2

555.9

556.0

556.1

556.2

556.3

556.9

556.6

720.0

696.1

696.8

696.0

585.3

585.4

585.5

585.6

\section{CodeType}

ICD-9 Diagnosis

ICD-9 Diagnosis

ICD-9 Diagnosis

ICD-9 Diagnosis

ICD-9 Diagnosis

ICD-9 Diagnosis

ICD-9 Diagnosis

ICD-9 Diagnosis

ICD-9 Diagnosis

ICD-9 Diagnosis

ICD-9 Diagnosis

ICD-9 Diagnosis

ICD-9 Diagnosis

ICD-9 Diagnosis

ICD-9 Diagnosis

ICD-9 Diagnosis

ICD-9 Diagnosis

ICD-9 Diagnosis

ICD-9 Diagnosis

ICD-9 Diagnosis

ICD-9 Diagnosis

ICD-9 Diagnosis

ICD-9 Diagnosis

ICD-9 Diagnosis

ICD-9 Diagnosis

ICD-9 Diagnosis

ICD-9 Diagnosis

ICD-9 Diagnosis

ICD-9 Diagnosis

ICD-9 Diagnosis

ICD-9 Diagnosis

ICD-9 Diagnosis

ICD-9 Diagnosis 
APPENDIX B Code Lists (continued)

\section{Category}

Immunocompromising conditions Immunocompromising conditions Immunocompromising conditions Immunocompromising conditions Immunocompromising conditions Immunocompromising conditions Immunocompromising conditions Immunocompromising conditions Immunocompromising conditions Immunocompromising conditions Immunocompromising conditions Immunocompromising conditions Immunocompromising conditions Immunocompromising conditions Immunocompromising conditions Immunocompromising conditions Immunocompromising conditions Immunocompromising conditions Immunocompromising conditions Immunocompromising conditions Immunocompromising conditions Immunocompromising conditions Immunocompromising conditions Immunocompromising conditions Immunocompromising conditions Immunocompromising conditions Immunocompromising conditions Immunocompromising conditions Immunocompromising conditions Immunocompromising conditions Immunocompromising conditions Immunocompromising conditions Immunocompromising conditions Immunocompromising conditions Immunocompromising conditions Immunocompromising conditions Immunocompromising conditions Immunocompromising conditions Immunocompromising conditions Immunocompromising conditions Immunocompromising conditions Immunocompromising conditions

\section{Description}

Chronic kidney disease

Chronic kidney disease

Chronic kidney disease

Chronic kidney disease

Chronic kidney disease

Chronic kidney disease

Chronic kidney disease

Chronic kidney disease

Chronic kidney disease

Chronic kidney disease

Chronic kidney disease

Chronic kidney disease

Chronic kidney disease

Chronic kidney disease

Chronic kidney disease

Chronic kidney disease

Chronic kidney disease

Chronic kidney disease

Chronic kidney disease

Chronic kidney disease

Chronic kidney disease

Chronic kidney disease

Chronic kidney disease

Chronic kidney disease

Chronic kidney disease

Chronic kidney disease

Chronic kidney disease

Chronic kidney disease

Chronic kidney disease

Chronic kidney disease

Chronic kidney disease

Chronic kidney disease

Chronic kidney disease

Chronic kidney disease

Chronic kidney disease

Chronic kidney disease

Chronic kidney disease

Chronic kidney disease

Chronic kidney disease

Chronic kidney disease

Chronic kidney disease

Chronic kidney disease

\section{Code}

90918

90919

90920

90921

90922

90923

90924

90925

90935

90937

90939

90940

90945

90947

90951

90952

90953

90954

90955

90956

90957

90958

90959

90960

90961

90962

90963

90964

90965

90966

90967

90968

90969

90970

90989

90993

90997

90999

39.27

39.42

39.43

39.95
CodeType

HCPCS

HCPCS

HCPCS

HCPCS

HCPCS

HCPCS

HCPCS

HCPCS

HCPCS

HCPCS

HCPCS

HCPCS

HCPCS

HCPCS

HCPCS

HCPCS

HCPCS

HCPCS

HCPCS

HCPCS

HCPCS

HCPCS

HCPCS

HCPCS

HCPCS

HCPCS

HCPCS

HCPCS

HCPCS

HCPCS

HCPCS

HCPCS

HCPCS

HCPCS

HCPCS

HCPCS

HCPCS

HCPCS

ICD-9 Procedure

ICD-9 Procedure

ICD-9 Procedure

ICD-9 Procedure

continued on next page 
APPENDIX B Code Lists (continued)

\section{Category}

Immunocompromising conditions Immunocompromising conditions Immunocompromising conditions Immunocompromising conditions Immunocompromising conditions Immunocompromising conditions Immunocompromising conditions Immunocompromising conditions Immunocompromising conditions Immunocompromising conditions Immunocompromising conditions Immunocompromising conditions Immunocompromising conditions Immunocompromising conditions Immunocompromising conditions Immunocompromising conditions Immunocompromising conditions Immunocompromising conditions Immunocompromising conditions Immunocompromising conditions Immunocompromising conditions Immunocompromising conditions Immunocompromising conditions Immunocompromising conditions Immunocompromising conditions Immunocompromising conditions Immunocompromising conditions Immunocompromising conditions Immunocompromising conditions Immunocompromising conditions Immunocompromising conditions Immunocompromising conditions Immunocompromising conditions Immunocompromising conditions Immunocompromising conditions Immunocompromising conditions Immunocompromising conditions Immunocompromising conditions Immunocompromising conditions Immunocompromising conditions Immunocompromising conditions Immunocompromising conditions

\section{Description}

Chronic liver disease

Chronic liver disease

Chronic liver disease

Chronic liver disease

Chronic liver disease

Chronic liver disease

HIV

HIV

HIV

HIV

HIV

HIV

Organ transplantation

Organ transplantation

Organ transplantation

Organ transplantation

Organ transplantation

Organ transplantation

Organ transplantation

Organ transplantation

Organ transplantation

Organ transplantation

Organ transplantation

Organ transplantation

Organ transplantation

Organ transplantation

Organ transplantation

Organ transplantation

Organ transplantation

Organ transplantation

Organ transplantation

Organ transplantation

Organ transplantation

Organ transplantation

Organ transplantation

Organ transplantation

Organ transplantation

Organ transplantation

Organ transplantation

Organ transplantation

Organ transplantation

Organ transplantation

\section{Code}

456.0

456.1

456.20

456.21

572.2

789.5

042

043

044

V08

795.71

079.53

V42.0

V42.1

V42.6

V42.7

V42.81

V42.82

V42.83

V42.84

32851

32852

32853

32854

33935

38240

38241

47135

47136

48554

48556

50340

50360

50365

50370

50380

33.5

33.6

37.5

41.0

50.5

52.8

\section{CodeType}

ICD-9 Diagnosis

ICD-9 Diagnosis

ICD-9 Diagnosis

ICD-9 Diagnosis

ICD-9 Diagnosis

ICD-9 Diagnosis

ICD-9 Diagnosis

ICD-9 Diagnosis

ICD-9 Diagnosis

ICD-9 Diagnosis

ICD-9 Diagnosis

ICD-9 Diagnosis

ICD-9 Diagnosis

ICD-9 Diagnosis

ICD-9 Diagnosis

ICD-9 Diagnosis

ICD-9 Diagnosis

ICD-9 Diagnosis

ICD-9 Diagnosis

ICD-9 Diagnosis

HCPCS

HCPCS

HCPCS

HCPCS

HCPCS

HCPCS

HCPCS

HCPCS

HCPCS

HCPCS

HCPCS

HCPCS

HCPCS

HCPCS

HCPCS

HCPCS

ICD-9 Procedure

ICD-9 Procedure

ICD-9 Procedure

ICD-9 Procedure

ICD-9 Procedure

ICD-9 Procedure

continued on next page 
APPENDIX B Code Lists (continued)

\section{Category}

Immunocompromising conditions

Immunocompromising conditions

Immunocompromising conditions

Non-melanoma cancer

Non-melanoma cancer

Non-melanoma cancer

Non-melanoma cancer

Non-melanoma cancer

Non-melanoma cancer

Non-melanoma cancer

Non-melanoma cancer

Non-melanoma cancer

Non-melanoma cancer

Non-melanoma cancer

Non-melanoma cancer

Non-melanoma cancer

Non-melanoma cancer

Non-melanoma cancer

Non-melanoma cancer

Non-melanoma cancer

Non-melanoma cancer

Non-melanoma cancer

Non-melanoma cancer

Non-melanoma cancer

Non-melanoma cancer

Non-melanoma cancer

Non-melanoma cancer

Non-melanoma cancer

Non-melanoma cancer

Non-melanoma cancer

Non-melanoma cancer

Non-melanoma cancer

Non-melanoma cancer

Non-melanoma cancer

Non-melanoma cancer

Non-melanoma cancer

Non-melanoma cancer

Non-melanoma cancer

Non-melanoma cancer

Non-melanoma cancer

Non-melanoma cancer

Non-melanoma cancer

\section{Description}

Organ transplantation

Organ transplantation

Organ transplantation

Non-melanoma cancer

Non-melanoma cancer

Non-melanoma cancer

Non-melanoma cancer

Non-melanoma cancer

Non-melanoma cancer

Non-melanoma cancer

Non-melanoma cancer

Non-melanoma cancer

Non-melanoma cancer

Non-melanoma cancer

Non-melanoma cancer

Non-melanoma cancer

Non-melanoma cancer

Non-melanoma cancer

Non-melanoma cancer

Non-melanoma cancer

Non-melanoma cancer

Non-melanoma cancer

Non-melanoma cancer

Non-melanoma cancer

Non-melanoma cancer

Non-melanoma cancer

Non-melanoma cancer

Non-melanoma cancer

Non-melanoma cancer

Non-melanoma cancer

Non-melanoma cancer

Non-melanoma cancer

Non-melanoma cancer

Non-melanoma cancer

Non-melanoma cancer

Non-melanoma cancer

Non-melanoma cancer

Non-melanoma cancer

Non-melanoma cancer

Non-melanoma cancer

Non-melanoma cancer

Non-melanoma cancer

\begin{tabular}{|c|c|}
\hline Code & CodeType \\
\hline 55.6 & ICD-9 Procedure \\
\hline 46.97 & ICD-9 Procedure \\
\hline 54.98 & ICD-9 Procedure \\
\hline 140 & ICD-9 Diagnosis \\
\hline 140 & ICD-9 Diagnosis \\
\hline $140 *$ & ICD-9 Diagnosis \\
\hline $140 * *$ & ICD-9 Diagnosis \\
\hline 141 & ICD-9 Diagnosis \\
\hline $141^{*}$ & ICD-9 Diagnosis \\
\hline $141 * *$ & ICD-9 Diagnosis \\
\hline 142 & ICD-9 Diagnosis \\
\hline $142 *$ & ICD-9 Diagnosis \\
\hline $142 * *$ & ICD-9 Diagnosis \\
\hline 143 & ICD-9 Diagnosis \\
\hline $143^{*}$ & ICD-9 Diagnosis \\
\hline $143 * *$ & ICD-9 Diagnosis \\
\hline 144 & ICD-9 Diagnosis \\
\hline $144^{*}$ & ICD-9 Diagnosis \\
\hline $144 * *$ & ICD-9 Diagnosis \\
\hline 145 & ICD-9 Diagnosis \\
\hline $145^{*}$ & ICD-9 Diagnosis \\
\hline $145 * *$ & ICD-9 Diagnosis \\
\hline 146 & ICD-9 Diagnosis \\
\hline $146^{*}$ & ICD-9 Diagnosis \\
\hline $146 * *$ & ICD-9 Diagnosis \\
\hline 147 & ICD-9 Diagnosis \\
\hline $147^{*}$ & ICD-9 Diagnosis \\
\hline $147 * *$ & ICD-9 Diagnosis \\
\hline 148 & ICD-9 Diagnosis \\
\hline $148^{*}$ & ICD-9 Diagnosis \\
\hline $148 * *$ & ICD-9 Diagnosis \\
\hline 149 & ICD-9 Diagnosis \\
\hline $149 *$ & ICD-9 Diagnosis \\
\hline $149 * *$ & ICD-9 Diagnosis \\
\hline 150 & ICD-9 Diagnosis \\
\hline $150 *$ & ICD-9 Diagnosis \\
\hline $150 * *$ & ICD-9 Diagnosis \\
\hline 151 & ICD-9 Diagnosis \\
\hline $151 *$ & ICD-9 Diagnosis \\
\hline $151 * *$ & ICD-9 Diagnosis \\
\hline 152 & ICD-9 Diagnosis \\
\hline $152^{*}$ & ICD-9 Diagnosis \\
\hline
\end{tabular}

continued on next page 
APPENDIX B Code Lists (continued)

\section{Category}

Non-melanoma cancer

Non-melanoma cancer

Non-melanoma cancer

Non-melanoma cancer

Non-melanoma cancer

Non-melanoma cancer

Non-melanoma cancer

Non-melanoma cancer

Non-melanoma cancer

Non-melanoma cancer

Non-melanoma cancer

Non-melanoma cancer

Non-melanoma cancer

Non-melanoma cancer

Non-melanoma cancer

Non-melanoma cancer

Non-melanoma cancer

Non-melanoma cancer

Non-melanoma cancer

Non-melanoma cancer

Non-melanoma cancer

Non-melanoma cancer

Non-melanoma cancer

Non-melanoma cancer

Non-melanoma cancer

Non-melanoma cancer

Non-melanoma cancer

Non-melanoma cancer

Non-melanoma cancer

Non-melanoma cancer

Non-melanoma cancer

Non-melanoma cancer

Non-melanoma cancer

Non-melanoma cancer

Non-melanoma cancer

Non-melanoma cancer

Non-melanoma cancer

Non-melanoma cancer

Non-melanoma cancer

Non-melanoma cancer

Non-melanoma cancer

Non-melanoma cancer

\section{Description}

Non-melanoma cancer

Non-melanoma cancer

Non-melanoma cancer

Non-melanoma cancer

Non-melanoma cancer

Non-melanoma cancer

Non-melanoma cancer

Non-melanoma cancer

Non-melanoma cancer

Non-melanoma cancer

Non-melanoma cancer

Non-melanoma cancer

Non-melanoma cancer

Non-melanoma cancer

Non-melanoma cancer

Non-melanoma cancer

Non-melanoma cancer

Non-melanoma cancer

Non-melanoma cancer

Non-melanoma cancer

Non-melanoma cancer

Non-melanoma cancer

Non-melanoma cancer

Non-melanoma cancer

Non-melanoma cancer

Non-melanoma cancer

Non-melanoma cancer

Non-melanoma cancer

Non-melanoma cancer

Non-melanoma cancer

Non-melanoma cancer

Non-melanoma cancer

Non-melanoma cancer

Non-melanoma cancer

Non-melanoma cancer

Non-melanoma cancer

Non-melanoma cancer

Non-melanoma cancer

Non-melanoma cancer

Non-melanoma cancer

Non-melanoma cancer

Non-melanoma cancer

\begin{tabular}{|c|c|}
\hline Code & CodeType \\
\hline $152 * *$ & ICD-9 Diagnosis \\
\hline 153 & ICD-9 Diagnosis \\
\hline $153^{*}$ & ICD-9 Diagnosis \\
\hline $153^{* *}$ & ICD-9 Diagnosis \\
\hline 154 & ICD-9 Diagnosis \\
\hline $154^{*}$ & ICD-9 Diagnosis \\
\hline $154^{* *}$ & ICD-9 Diagnosis \\
\hline 155 & ICD-9 Diagnosis \\
\hline $155^{*}$ & ICD-9 Diagnosis \\
\hline $155^{* *}$ & ICD-9 Diagnosis \\
\hline 156 & ICD-9 Diagnosis \\
\hline $156^{*}$ & ICD-9 Diagnosis \\
\hline $156 * *$ & ICD-9 Diagnosis \\
\hline 157 & ICD-9 Diagnosis \\
\hline $157^{*}$ & ICD-9 Diagnosis \\
\hline $157^{* *}$ & ICD-9 Diagnosis \\
\hline 158 & ICD-9 Diagnosis \\
\hline $158^{*}$ & ICD-9 Diagnosis \\
\hline $158^{* *}$ & ICD-9 Diagnosis \\
\hline 159 & ICD-9 Diagnosis \\
\hline $159 *$ & ICD-9 Diagnosis \\
\hline $159 * *$ & ICD-9 Diagnosis \\
\hline 160 & ICD-9 Diagnosis \\
\hline $160 *$ & ICD-9 Diagnosis \\
\hline $160^{* *}$ & ICD-9 Diagnosis \\
\hline 161 & ICD-9 Diagnosis \\
\hline $161^{*}$ & ICD-9 Diagnosis \\
\hline $161 * *$ & ICD-9 Diagnosis \\
\hline 162 & ICD-9 Diagnosis \\
\hline $162^{*}$ & ICD-9 Diagnosis \\
\hline $162 * *$ & ICD-9 Diagnosis \\
\hline 163 & ICD-9 Diagnosis \\
\hline $163^{*}$ & ICD-9 Diagnosis \\
\hline $163 * *$ & ICD-9 Diagnosis \\
\hline 164 & ICD-9 Diagnosis \\
\hline $164 *$ & ICD-9 Diagnosis \\
\hline $164 * *$ & ICD-9 Diagnosis \\
\hline 165 & ICD-9 Diagnosis \\
\hline $165^{*}$ & ICD-9 Diagnosis \\
\hline $165^{* *}$ & ICD-9 Diagnosis \\
\hline 170.0 & ICD-9 Diagnosis \\
\hline 170.1 & ICD-9 Diagnosis \\
\hline
\end{tabular}

continued on next page 
APPENDIX B Code Lists (continued)

\section{Category}

Non-melanoma cancer

Non-melanoma cancer

Non-melanoma cancer

Non-melanoma cancer

Non-melanoma cancer

Non-melanoma cancer

Non-melanoma cancer

Non-melanoma cancer

Non-melanoma cancer

Non-melanoma cancer

Non-melanoma cancer

Non-melanoma cancer

Non-melanoma cancer

Non-melanoma cancer

Non-melanoma cancer

Non-melanoma cancer

Non-melanoma cancer

Non-melanoma cancer

Non-melanoma cancer

Non-melanoma cancer

Non-melanoma cancer

Non-melanoma cancer

Non-melanoma cancer

Non-melanoma cancer

Non-melanoma cancer

Non-melanoma cancer

Non-melanoma cancer

Non-melanoma cancer

Non-melanoma cancer

Non-melanoma cancer

Non-melanoma cancer

Non-melanoma cancer

Non-melanoma cancer

Non-melanoma cancer

Non-melanoma cancer

Non-melanoma cancer

Non-melanoma cancer

Non-melanoma cancer

Non-melanoma cancer

Non-melanoma cancer

Non-melanoma cancer

Non-melanoma cancer

\section{Description}

Non-melanoma cancer

Non-melanoma cancer

Non-melanoma cancer

Non-melanoma cancer

Non-melanoma cancer

Non-melanoma cancer

Non-melanoma cancer

Non-melanoma cancer

Non-melanoma cancer

Non-melanoma cancer

Non-melanoma cancer

Non-melanoma cancer

Non-melanoma cancer

Non-melanoma cancer

Non-melanoma cancer

Non-melanoma cancer

Non-melanoma cancer

Non-melanoma cancer

Non-melanoma cancer

Non-melanoma cancer

Non-melanoma cancer

Non-melanoma cancer

Non-melanoma cancer

Non-melanoma cancer

Non-melanoma cancer

Non-melanoma cancer

Non-melanoma cancer

Non-melanoma cancer

Non-melanoma cancer

Non-melanoma cancer

Non-melanoma cancer

Non-melanoma cancer

Non-melanoma cancer

Non-melanoma cancer

Non-melanoma cancer

Non-melanoma cancer

Non-melanoma cancer

Non-melanoma cancer

Non-melanoma cancer

Non-melanoma cancer

Non-melanoma cancer

Non-melanoma cancer

\section{Code}

170.2

170.3

170.4

170.5

170.6

170.7

170.8

170.9

171.0

171.2

171.3

171.4

171.5

171.6

171.7

171.8

171.9

172.0

172.1

172.2

172.3

172.4

172.5

172.6

172.7

172.8

172.9

174.0

174.1

174.2

174.3

174.4

174.5

174.6

174.8

174.9

175

176

179

180

181

182
CodeType

ICD-9 Diagnosis

ICD-9 Diagnosis

ICD-9 Diagnosis

ICD-9 Diagnosis

ICD-9 Diagnosis

ICD-9 Diagnosis

ICD-9 Diagnosis

ICD-9 Diagnosis

ICD-9 Diagnosis

ICD-9 Diagnosis

ICD-9 Diagnosis

ICD-9 Diagnosis

ICD-9 Diagnosis

ICD-9 Diagnosis

ICD-9 Diagnosis

ICD-9 Diagnosis

ICD-9 Diagnosis

ICD-9 Diagnosis

ICD-9 Diagnosis

ICD-9 Diagnosis

ICD-9 Diagnosis

ICD-9 Diagnosis

ICD-9 Diagnosis

ICD-9 Diagnosis

ICD-9 Diagnosis

ICD-9 Diagnosis

ICD-9 Diagnosis

ICD-9 Diagnosis

ICD-9 Diagnosis

ICD-9 Diagnosis

ICD-9 Diagnosis

ICD-9 Diagnosis

ICD-9 Diagnosis

ICD-9 Diagnosis

ICD-9 Diagnosis

ICD-9 Diagnosis

ICD-9 Diagnosis

ICD-9 Diagnosis

ICD-9 Diagnosis

ICD-9 Diagnosis

ICD-9 Diagnosis

ICD-9 Diagnosis

continued on next page 
APPENDIX B Code Lists (continued)

\section{Category}

Non-melanoma cancer

Non-melanoma cancer

Non-melanoma cancer

Non-melanoma cancer

Non-melanoma cancer

Non-melanoma cancer

Non-melanoma cancer

Non-melanoma cancer

Non-melanoma cancer

Non-melanoma cancer

Non-melanoma cancer

Non-melanoma cancer

Non-melanoma cancer

Non-melanoma cancer

Non-melanoma cancer

Non-melanoma cancer

Non-melanoma cancer

Non-melanoma cancer

Non-melanoma cancer

Non-melanoma cancer

Non-melanoma cancer

Non-melanoma cancer

Non-melanoma cancer

Non-melanoma cancer

Non-melanoma cancer

Non-melanoma cancer

Non-melanoma cancer

Non-melanoma cancer

Non-melanoma cancer

Non-melanoma cancer

Non-melanoma cancer

Non-melanoma cancer

Non-melanoma cancer

Non-melanoma cancer

Non-melanoma cancer

Non-melanoma cancer

Non-melanoma cancer

Non-melanoma cancer

Non-melanoma cancer

Non-melanoma cancer

Non-melanoma cancer

Non-melanoma cancer

\section{Description}

Non-melanoma cancer

Non-melanoma cancer

Non-melanoma cancer

Non-melanoma cancer

Non-melanoma cancer

Non-melanoma cancer

Non-melanoma cancer

Non-melanoma cancer

Non-melanoma cancer

Non-melanoma cancer

Non-melanoma cancer

Non-melanoma cancer

Non-melanoma cancer

Non-melanoma cancer

Non-melanoma cancer

Non-melanoma cancer

Non-melanoma cancer

Non-melanoma cancer

Non-melanoma cancer

Non-melanoma cancer

Non-melanoma cancer

Non-melanoma cancer

Non-melanoma cancer

Non-melanoma cancer

Non-melanoma cancer

Non-melanoma cancer

Non-melanoma cancer

Non-melanoma cancer

Non-melanoma cancer

Non-melanoma cancer

Non-melanoma cancer

Non-melanoma cancer

Non-melanoma cancer

Non-melanoma cancer

Non-melanoma cancer

Non-melanoma cancer

Non-melanoma cancer

Non-melanoma cancer

Non-melanoma cancer

Non-melanoma cancer

Non-melanoma cancer

Non-melanoma cancer

\section{Code}

183

184

185

186

187

188

189

190

191

192

193

194

195

196

197

198

199

200

201

202

203

204

205

206

207

208

$175 *$

$176^{*}$

$179 *$

$180 *$

$181 *$

$182 *$

183*

$184 *$

$185^{*}$

186*

$187^{*}$

188*

$189 *$

190*

191*

192*
CodeType

ICD-9 Diagnosis

ICD-9 Diagnosis

ICD-9 Diagnosis

ICD-9 Diagnosis

ICD-9 Diagnosis

ICD-9 Diagnosis

ICD-9 Diagnosis

ICD-9 Diagnosis

ICD-9 Diagnosis

ICD-9 Diagnosis

ICD-9 Diagnosis

ICD-9 Diagnosis

ICD-9 Diagnosis

ICD-9 Diagnosis

ICD-9 Diagnosis

ICD-9 Diagnosis

ICD-9 Diagnosis

ICD-9 Diagnosis

ICD-9 Diagnosis

ICD-9 Diagnosis

ICD-9 Diagnosis

ICD-9 Diagnosis

ICD-9 Diagnosis

ICD-9 Diagnosis

ICD-9 Diagnosis

ICD-9 Diagnosis

ICD-9 Diagnosis

ICD-9 Diagnosis

ICD-9 Diagnosis

ICD-9 Diagnosis

ICD-9 Diagnosis

ICD-9 Diagnosis

ICD-9 Diagnosis

ICD-9 Diagnosis

ICD-9 Diagnosis

ICD-9 Diagnosis

ICD-9 Diagnosis

ICD-9 Diagnosis

ICD-9 Diagnosis

ICD-9 Diagnosis

ICD-9 Diagnosis

ICD-9 Diagnosis

continued on next page 
APPENDIX B Code Lists (continued)

\section{Category}

Non-melanoma cancer

Non-melanoma cancer

Non-melanoma cancer

Non-melanoma cancer

Non-melanoma cancer

Non-melanoma cancer

Non-melanoma cancer

Non-melanoma cancer

Non-melanoma cancer

Non-melanoma cancer

Non-melanoma cancer

Non-melanoma cancer

Non-melanoma cancer

Non-melanoma cancer

Non-melanoma cancer

Non-melanoma cancer

Non-melanoma cancer

Non-melanoma cancer

Non-melanoma cancer

Non-melanoma cancer

Non-melanoma cancer

Non-melanoma cancer

Non-melanoma cancer

Non-melanoma cancer

Non-melanoma cancer

Non-melanoma cancer

Non-melanoma cancer

Non-melanoma cancer

Non-melanoma cancer

Non-melanoma cancer

Non-melanoma cancer

Non-melanoma cancer

Non-melanoma cancer

Non-melanoma cancer

Non-melanoma cancer

Non-melanoma cancer

Non-melanoma cancer

Non-melanoma cancer

Non-melanoma cancer

Non-melanoma cancer

Non-melanoma cancer

Non-melanoma cancer

\section{Description}

Non-melanoma cancer

Non-melanoma cancer

Non-melanoma cancer

Non-melanoma cancer

Non-melanoma cancer

Non-melanoma cancer

Non-melanoma cancer

Non-melanoma cancer

Non-melanoma cancer

Non-melanoma cancer

Non-melanoma cancer

Non-melanoma cancer

Non-melanoma cancer

Non-melanoma cancer

Non-melanoma cancer

Non-melanoma cancer

Non-melanoma cancer

Non-melanoma cancer

Non-melanoma cancer

Non-melanoma cancer

Non-melanoma cancer

Non-melanoma cancer

Non-melanoma cancer

Non-melanoma cancer

Non-melanoma cancer

Non-melanoma cancer

Non-melanoma cancer

Non-melanoma cancer

Non-melanoma cancer

Non-melanoma cancer

Non-melanoma cancer

Non-melanoma cancer

Non-melanoma cancer

Non-melanoma cancer

Non-melanoma cancer

Non-melanoma cancer

Non-melanoma cancer

Non-melanoma cancer

Non-melanoma cancer

Non-melanoma cancer

Non-melanoma cancer

Non-melanoma cancer
Code
193*
194*
$195 *$
196*
197*
198*
199*
200*
201*
$202 *$
203*
204*
205*
206*
$207 *$
208*
$175 * *$
$176 * *$
$179 * *$
$180 * *$
$181 * *$
$182 * *$
$183 * *$
$184 * *$
$185 * *$
$186 * *$
$187 * *$
$188^{* *}$
$189 * *$
190**
$191 * *$
$192 * *$
$193 * *$
$194 * *$
$195 * *$
$196 * *$
$197 * *$
$198 * *$
$199 * *$
200**
201**
$202 * *$
CodeType
ICD-9 Diagnosis
ICD-9 Diagnosis
ICD-9 Diagnosis
ICD-9 Diagnosis
ICD-9 Diagnosis
ICD-9 Diagnosis
ICD-9 Diagnosis
ICD-9 Diagnosis
ICD-9 Diagnosis
ICD-9 Diagnosis
ICD-9 Diagnosis
ICD-9 Diagnosis
ICD-9 Diagnosis
ICD-9 Diagnosis
ICD-9 Diagnosis
ICD-9 Diagnosis
ICD-9 Diagnosis
ICD-9 Diagnosis
ICD-9 Diagnosis
ICD-9 Diagnosis
ICD-9 Diagnosis
ICD-9 Diagnosis
ICD-9 Diagnosis
ICD-9 Diagnosis
ICD-9 Diagnosis
ICD-9 Diagnosis
ICD-9 Diagnosis
ICD-9 Diagnosis
ICD-9 Diagnosis
ICD-9 Diagnosis
ICD-9 Diagnosis
ICD-9 Diagnosis
ICD-9 Diagnosis
ICD-9 Diagnosis
ICD-9 Diagnosis
ICD-9 Diagnosis
ICD-9 Diagnosis
ICD-9 Diagnosis
ICD-9 Diagnosis
ICD-9 Diagnosis
ICD-9 Diagnosis
ICD-9 Diagnosis

continued on next page 
APPENDIX B Code Lists (continued)

\section{Category}

Non-melanoma cancer

Non-melanoma cancer

Non-melanoma cancer

Non-melanoma cancer

Non-melanoma cancer

Non-melanoma cancer

Non-melanoma cancer

Non-melanoma cancer

Non-melanoma cancer

Non-melanoma cancer

Non-melanoma cancer

Non-melanoma cancer

Non-melanoma cancer

Non-melanoma cancer

Non-melanoma cancer

Non-melanoma cancer

Non-melanoma cancer

Non-melanoma cancer

Non-melanoma cancer

Non-melanoma cancer

Non-melanoma cancer

Non-melanoma cancer

Non-melanoma cancer

Non-melanoma cancer

Non-melanoma cancer

Non-melanoma cancer

Non-melanoma cancer

Non-melanoma cancer

Non-melanoma cancer

Non-melanoma cancer

Non-melanoma cancer

Non-melanoma cancer

Non-melanoma cancer

Non-melanoma cancer

Non-melanoma cancer

Non-melanoma cancer

Non-melanoma cancer

Non-melanoma cancer

Non-melanoma cancer

Non-melanoma cancer

Non-melanoma cancer

Non-melanoma cancer

\section{Description}

Non-melanoma cancer

Non-melanoma cancer

Non-melanoma cancer

Non-melanoma cancer

Non-melanoma cancer

Non-melanoma cancer

Non-melanoma cancer

Non-melanoma cancer

Non-melanoma cancer

Non-melanoma cancer

Non-melanoma cancer

Non-melanoma cancer

Non-melanoma cancer

Non-melanoma cancer

Non-melanoma cancer

Non-melanoma cancer

Non-melanoma cancer

Non-melanoma cancer

Non-melanoma cancer

Non-melanoma cancer

Non-melanoma cancer

Non-melanoma cancer

Non-melanoma cancer

Non-melanoma cancer

Non-melanoma cancer

Non-melanoma cancer

Non-melanoma cancer

Non-melanoma cancer

Non-melanoma cancer

Non-melanoma cancer

Non-melanoma cancer

Non-melanoma cancer

Non-melanoma cancer

Non-melanoma cancer

Non-melanoma cancer

Non-melanoma cancer

Non-melanoma cancer

Non-melanoma cancer

Non-melanoma cancer

Non-melanoma cancer

Non-melanoma cancer

Non-melanoma cancer
Code
203**
204**
205**
$206 * *$
$207 * *$
$208 * *$
169**
$175 *$
$175 * *$
176*
$176 * *$
179*
179**
180*
180 **
181*
$181 * *$
$182 *$
$182 * *$
183*
$183^{* *}$
184*
$184 * *$
185*
$185^{* *}$
186*
$186 * *$
$187^{*}$
$187^{* *}$
188*
$188^{* *}$
189*
$189 * *$
190*
$190 * *$
191*
$191^{* *}$
$192 *$
$192 * *$
193*
193**
194*

\section{CodeType}
ICD-9 Diagnosis
ICD-9 Diagnosis
ICD-9 Diagnosis
ICD-9 Diagnosis
ICD-9 Diagnosis
ICD-9 Diagnosis
ICD-9 Diagnosis
ICD-9 Diagnosis
ICD-9 Diagnosis
ICD-9 Diagnosis
ICD-9 Diagnosis
ICD-9 Diagnosis
ICD-9 Diagnosis
ICD-9 Diagnosis
ICD-9 Diagnosis
ICD-9 Diagnosis
ICD-9 Diagnosis
ICD-9 Diagnosis
ICD-9 Diagnosis
ICD-9 Diagnosis
ICD-9 Diagnosis
ICD-9 Diagnosis
ICD-9 Diagnosis
ICD-9 Diagnosis
ICD-9 Diagnosis
ICD-9 Diagnosis
ICD-9 Diagnosis
ICD-9 Diagnosis
ICD-9 Diagnosis
ICD-9 Diagnosis
ICD-9 Diagnosis
ICD-9 Diagnosis
ICD-9 Diagnosis
ICD-9 Diagnosis
ICD-9 Diagnosis
ICD-9 Diagnosis
ICD-9 Diagnosis
ICD-9 Diagnosis
ICD-9 Diagnosis
ICD-9 Diagnosis
ICD-9 Diagnosis
ICD-9 Diagnosis

continued on next page 
APPENDIX B Code Lists (continued)

\section{Category}

Non-melanoma cancer

Non-melanoma cancer

Non-melanoma cancer

Non-melanoma cancer

Non-melanoma cancer

Non-melanoma cancer

Non-melanoma cancer

Non-melanoma cancer

Non-melanoma cancer

Non-melanoma cancer

Non-melanoma cancer

Non-melanoma cancer

Non-melanoma cancer

Non-melanoma cancer

Non-melanoma cancer

Non-melanoma cancer

Non-melanoma cancer

Non-melanoma cancer

Non-melanoma cancer

Non-melanoma cancer

Non-melanoma cancer

Non-melanoma cancer

Non-melanoma cancer

Non-melanoma cancer

Non-melanoma cancer

Non-melanoma cancer

Non-melanoma cancer

Non-melanoma cancer

Non-melanoma cancer

Serious infection

Serious infection

Serious infection

Serious infection

Serious infection

Serious infection

Serious infection

Serious infection

Serious infection

Serious infection

Serious infection

Serious infection

Serious infection

\section{Description}

Non-melanoma cancer

Non-melanoma cancer

Non-melanoma cancer

Non-melanoma cancer

Non-melanoma cancer

Non-melanoma cancer

Non-melanoma cancer

Non-melanoma cancer

Non-melanoma cancer

Non-melanoma cancer

Non-melanoma cancer

Non-melanoma cancer

Non-melanoma cancer

Non-melanoma cancer

Non-melanoma cancer

Non-melanoma cancer

Non-melanoma cancer

Non-melanoma cancer

Non-melanoma cancer

Non-melanoma cancer

Non-melanoma cancer

Non-melanoma cancer

Non-melanoma cancer

Non-melanoma cancer

Non-melanoma cancer

Non-melanoma cancer

Non-melanoma cancer

Non-melanoma cancer

Non-melanoma cancer

Appendicitis

Appendicitis

Appendicitis

Appendicitis

Appendicitis

Appendicitis

Appendicitis

Infectious arthritis

Infectious arthritis

Infectious arthritis

Infectious arthritis

Infectious arthritis

Infectious arthritis

\section{Code}

$194 * *$

195*

$195 * *$

196*

$196 * *$

197*

$197 * *$

198*

$198 * *$

199*

199**

200*

$200 * *$

201*

$201 * *$

202*

$202 *$

203*

$203 * *$

204*

$204 * *$

$205 *$

205**

206*

$206 * *$

207*

$207 * *$

208*

$208 * *$

540.0

540.1

540.9

541

542

543.0

543.9

003.23

003.24

026.1

036.82

056.71

711.00
CodeType

ICD-9 Diagnosis

ICD-9 Diagnosis

ICD-9 Diagnosis

ICD-9 Diagnosis

ICD-9 Diagnosis

ICD-9 Diagnosis

ICD-9 Diagnosis

ICD-9 Diagnosis

ICD-9 Diagnosis

ICD-9 Diagnosis

ICD-9 Diagnosis

ICD-9 Diagnosis

ICD-9 Diagnosis

ICD-9 Diagnosis

ICD-9 Diagnosis

ICD-9 Diagnosis

ICD-9 Diagnosis

ICD-9 Diagnosis

ICD-9 Diagnosis

ICD-9 Diagnosis

ICD-9 Diagnosis

ICD-9 Diagnosis

ICD-9 Diagnosis

ICD-9 Diagnosis

ICD-9 Diagnosis

ICD-9 Diagnosis

ICD-9 Diagnosis

ICD-9 Diagnosis

ICD-9 Diagnosis

ICD-9 Diagnosis

ICD-9 Diagnosis

ICD-9 Diagnosis

ICD-9 Diagnosis

ICD-9 Diagnosis

ICD-9 Diagnosis

ICD-9 Diagnosis

ICD-9 Diagnosis

ICD-9 Diagnosis

ICD-9 Diagnosis

ICD-9 Diagnosis

ICD-9 Diagnosis

ICD-9 Diagnosis

continued on next page 
APPENDIX B Code Lists (continued)

\section{Category}

Serious infection

Serious infection

Serious infection

Serious infection

Serious infection

Serious infection

Serious infection

Serious infection

Serious infection

Serious infection

Serious infection

Serious infection

Serious infection

Serious infection

Serious infection

Serious infection

Serious infection

Serious infection

Serious infection

Serious infection

Serious infection

Serious infection

Serious infection

Serious infection

Serious infection

Serious infection

Serious infection

Serious infection

Serious infection

Serious infection

Serious infection

Serious infection

Serious infection

Serious infection

Serious infection

Serious infection

Serious infection

Serious infection

Serious infection

Serious infection

Serious infection

Serious infection

\section{Description}

Infectious arthritis

Infectious arthritis

Infectious arthritis

Infectious arthritis

Infectious arthritis

Infectious arthritis

Infectious arthritis

Infectious arthritis

Infectious arthritis

Infectious arthritis

Infectious arthritis

Infectious arthritis

Infectious arthritis

Infectious arthritis

Infectious arthritis

Infectious arthritis

Infectious arthritis

Infectious arthritis

Infectious arthritis

Infectious arthritis

Infectious arthritis

Infectious arthritis

Infectious arthritis

Infectious arthritis

Infectious arthritis

Infectious arthritis

Infectious arthritis

Infectious arthritis

Infectious arthritis

Infectious arthritis

Infectious arthritis

Infectious arthritis

Infectious arthritis

Infectious arthritis

Infectious arthritis

Infectious arthritis

Infectious arthritis

Infectious arthritis

Infectious arthritis

Infectious arthritis

Infectious arthritis

Infectious arthritis
Code

711.01

711.02

711.03

711.04

711.05

711.06

711.07

711.08

711.09

711.10

711.11

711.12

711.13

711.14

711.15

711.16

711.17

711.18

711.19

711.20

711.21

711.22

711.23

711.24

711.25

711.26

711.27

711.28

711.29

711.30

711.31

711.32

711.33

711.34

711.35

711.36

711.37

711.38

711.39

711.40

711.41

711.42

\section{CodeType}

ICD-9 Diagnosis

ICD-9 Diagnosis

ICD-9 Diagnosis

ICD-9 Diagnosis

ICD-9 Diagnosis

ICD-9 Diagnosis

ICD-9 Diagnosis

ICD-9 Diagnosis

ICD-9 Diagnosis

ICD-9 Diagnosis

ICD-9 Diagnosis

ICD-9 Diagnosis

ICD-9 Diagnosis

ICD-9 Diagnosis

ICD-9 Diagnosis

ICD-9 Diagnosis

ICD-9 Diagnosis

ICD-9 Diagnosis

ICD-9 Diagnosis

ICD-9 Diagnosis

ICD-9 Diagnosis

ICD-9 Diagnosis

ICD-9 Diagnosis

ICD-9 Diagnosis

ICD-9 Diagnosis

ICD-9 Diagnosis

ICD-9 Diagnosis

ICD-9 Diagnosis

ICD-9 Diagnosis

ICD-9 Diagnosis

ICD-9 Diagnosis

ICD-9 Diagnosis

ICD-9 Diagnosis

ICD-9 Diagnosis

ICD-9 Diagnosis

ICD-9 Diagnosis

ICD-9 Diagnosis

ICD-9 Diagnosis

ICD-9 Diagnosis

ICD-9 Diagnosis

ICD-9 Diagnosis

ICD-9 Diagnosis

continued on next page 
APPENDIX B Code Lists (continued)

\section{Category}

Serious infection

Serious infection

Serious infection

Serious infection

Serious infection

Serious infection

Serious infection

Serious infection

Serious infection

Serious infection

Serious infection

Serious infection

Serious infection

Serious infection

Serious infection

Serious infection

Serious infection

Serious infection

Serious infection

Serious infection

Serious infection

Serious infection

Serious infection

Serious infection

Serious infection

Serious infection

Serious infection

Serious infection

Serious infection

Serious infection

Serious infection

Serious infection

Serious infection

Serious infection

Serious infection

Serious infection

Serious infection

Serious infection

Serious infection

Serious infection

Serious infection

Serious infection

\section{Description}

Infectious arthritis

Infectious arthritis

Infectious arthritis

Infectious arthritis

Infectious arthritis

Infectious arthritis

Infectious arthritis

Infectious arthritis

Infectious arthritis

Infectious arthritis

Infectious arthritis

Infectious arthritis

Infectious arthritis

Infectious arthritis

Infectious arthritis

Infectious arthritis

Infectious arthritis

Infectious arthritis

Infectious arthritis

Infectious arthritis

Infectious arthritis

Infectious arthritis

Infectious arthritis

Infectious arthritis

Infectious arthritis

Infectious arthritis

Infectious arthritis

Infectious arthritis

Infectious arthritis

Infectious arthritis

Infectious arthritis

Infectious arthritis

Infectious arthritis

Infectious arthritis

Infectious arthritis

Infectious arthritis

Infectious arthritis

Infectious arthritis

Infectious arthritis

Infectious arthritis

Infectious arthritis

Infectious arthritis

\section{Code}

711.43

711.44

711.45

711.46

711.47

711.48

711.49

711.50

711.51

711.52

711.53

711.54

711.55

711.56

711.57

711.58

711.59

711.60

711.61

711.62

711.63

711.64

711.65

711.66

711.67

711.68

711.69

711.70

711.71

711.72

711.73

711.74

711.75

711.76

711.77

711.78

711.79

711.80

711.81

711.82

711.83

711.84
CodeType

ICD-9 Diagnosis

ICD-9 Diagnosis

ICD-9 Diagnosis

ICD-9 Diagnosis

ICD-9 Diagnosis

ICD-9 Diagnosis

ICD-9 Diagnosis

ICD-9 Diagnosis

ICD-9 Diagnosis

ICD-9 Diagnosis

ICD-9 Diagnosis

ICD-9 Diagnosis

ICD-9 Diagnosis

ICD-9 Diagnosis

ICD-9 Diagnosis

ICD-9 Diagnosis

ICD-9 Diagnosis

ICD-9 Diagnosis

ICD-9 Diagnosis

ICD-9 Diagnosis

ICD-9 Diagnosis

ICD-9 Diagnosis

ICD-9 Diagnosis

ICD-9 Diagnosis

ICD-9 Diagnosis

ICD-9 Diagnosis

ICD-9 Diagnosis

ICD-9 Diagnosis

ICD-9 Diagnosis

ICD-9 Diagnosis

ICD-9 Diagnosis

ICD-9 Diagnosis

ICD-9 Diagnosis

ICD-9 Diagnosis

ICD-9 Diagnosis

ICD-9 Diagnosis

ICD-9 Diagnosis

ICD-9 Diagnosis

ICD-9 Diagnosis

ICD-9 Diagnosis

ICD-9 Diagnosis

ICD-9 Diagnosis

continued on next page 
APPENDIX B Code Lists (continued)

\section{Category}

Serious infection

Serious infection

Serious infection

Serious infection

Serious infection

Serious infection

Serious infection

Serious infection

Serious infection

Serious infection

Serious infection

Serious infection

Serious infection

Serious infection

Serious infection

Serious infection

Serious infection

Serious infection

Serious infection

Serious infection

Serious infection

Serious infection

Serious infection

Serious infection

Serious infection

Serious infection

Serious infection

Serious infection

Serious infection

Serious infection

Serious infection

Serious infection

Serious infection

Serious infection

Serious infection

Serious infection

Serious infection

Serious infection

Serious infection

Serious infection

Serious infection

Serious infection

\section{Description}

Infectious arthritis

Infectious arthritis

Infectious arthritis

Infectious arthritis

Infectious arthritis

Infectious arthritis

Infectious arthritis

Infectious arthritis

Infectious arthritis

Infectious arthritis

Infectious arthritis

Infectious arthritis

Infectious arthritis

Infectious arthritis

Infectious arthritis

Infectious arthritis

Infectious arthritis

Infectious arthritis

Infectious arthritis

Infectious arthritis

Infectious arthritis

Infectious arthritis

Infectious arthritis

Infectious arthritis

Infectious arthritis

Infectious arthritis

Infectious arthritis

Infectious arthritis

Infectious arthritis

Infectious arthritis

Infectious arthritis

Infectious arthritis

Infectious arthritis

Infectious arthritis

Infectious arthritis

Infectious arthritis

Infectious arthritis

Infectious arthritis

Infectious arthritis

Infectious arthritis

Infectious arthritis

Infectious arthritis

\section{Code}

711.85

711.86

711.87

711.88

711.89

711.90

711.91

711.92

711.93

711.94

711.95

711.96

711.97

711.98

711.99

730.00

730.01

730.02

730.03

730.04

730.05

730.06

730.07

730.08

730.09

730.10

730.11

730.12

730.13

730.14

730.15

730.16

730.17

730.18

730.19

730.20

730.21

730.22

730.23

730.24

730.25

730.26
CodeType

ICD-9 Diagnosis

ICD-9 Diagnosis

ICD-9 Diagnosis

ICD-9 Diagnosis

ICD-9 Diagnosis

ICD-9 Diagnosis

ICD-9 Diagnosis

ICD-9 Diagnosis

ICD-9 Diagnosis

ICD-9 Diagnosis

ICD-9 Diagnosis

ICD-9 Diagnosis

ICD-9 Diagnosis

ICD-9 Diagnosis

ICD-9 Diagnosis

ICD-9 Diagnosis

ICD-9 Diagnosis

ICD-9 Diagnosis

ICD-9 Diagnosis

ICD-9 Diagnosis

ICD-9 Diagnosis

ICD-9 Diagnosis

ICD-9 Diagnosis

ICD-9 Diagnosis

ICD-9 Diagnosis

ICD-9 Diagnosis

ICD-9 Diagnosis

ICD-9 Diagnosis

ICD-9 Diagnosis

ICD-9 Diagnosis

ICD-9 Diagnosis

ICD-9 Diagnosis

ICD-9 Diagnosis

ICD-9 Diagnosis

ICD-9 Diagnosis

ICD-9 Diagnosis

ICD-9 Diagnosis

ICD-9 Diagnosis

ICD-9 Diagnosis

ICD-9 Diagnosis

ICD-9 Diagnosis

ICD-9 Diagnosis

continued on next page 
APPENDIX B Code Lists (continued)

\section{Category}

Serious infection

Serious infection

Serious infection

Serious infection

Serious infection

Serious infection

Serious infection

Serious infection

Serious infection

Serious infection

Serious infection

Serious infection

Serious infection

Serious infection

Serious infection

Serious infection

Serious infection

Serious infection

Serious infection

Serious infection

Serious infection

Serious infection

Serious infection

Serious infection

Serious infection

Serious infection

Serious infection

Serious infection

Serious infection

Serious infection

Serious infection

Serious infection

Serious infection

Serious infection

Serious infection

Serious infection

Serious infection

Serious infection

Serious infection

Serious infection

Serious infection

Serious infection

\section{Description}

Infectious arthritis

Infectious arthritis

Infectious arthritis

Infectious arthritis

Infectious arthritis

Infectious arthritis

Infectious arthritis

Infectious arthritis

Infectious arthritis

Infectious arthritis

Infectious arthritis

Infectious arthritis

Infectious arthritis

Infectious arthritis

Infectious arthritis

Infectious arthritis

Infectious arthritis

Infectious arthritis

Infectious arthritis

Infectious arthritis

Infectious arthritis

Infectious arthritis

Infectious arthritis

Infectious arthritis

Infectious arthritis

Infectious arthritis

Infectious arthritis

Infectious arthritis

Infectious arthritis

Infectious arthritis

Infectious arthritis

Infectious arthritis

Infectious arthritis

Infectious arthritis

Infectious arthritis

Infectious arthritis

Infectious arthritis

Infectious arthritis

Infectious arthritis

Infectious arthritis

Infectious arthritis

Infectious arthritis

\section{Code}

730.27

730.28

730.29

730.30

730.31

730.32

730.33

730.34

730.35

730.36

730.37

730.38

730.39

730.70

730.71

730.72

730.73

730.74

730.75

730.76

730.77

730.78

730.79

730.80

730.81

730.82

730.83

730.84

730.85

730.86

730.87

730.88

730.89

730.90

730.91

730.92

730.93

730.94

730.95

730.96

730.97

730.98
CodeType

ICD-9 Diagnosis

ICD-9 Diagnosis

ICD-9 Diagnosis

ICD-9 Diagnosis

ICD-9 Diagnosis

ICD-9 Diagnosis

ICD-9 Diagnosis

ICD-9 Diagnosis

ICD-9 Diagnosis

ICD-9 Diagnosis

ICD-9 Diagnosis

ICD-9 Diagnosis

ICD-9 Diagnosis

ICD-9 Diagnosis

ICD-9 Diagnosis

ICD-9 Diagnosis

ICD-9 Diagnosis

ICD-9 Diagnosis

ICD-9 Diagnosis

ICD-9 Diagnosis

ICD-9 Diagnosis

ICD-9 Diagnosis

ICD-9 Diagnosis

ICD-9 Diagnosis

ICD-9 Diagnosis

ICD-9 Diagnosis

ICD-9 Diagnosis

ICD-9 Diagnosis

ICD-9 Diagnosis

ICD-9 Diagnosis

ICD-9 Diagnosis

ICD-9 Diagnosis

ICD-9 Diagnosis

ICD-9 Diagnosis

ICD-9 Diagnosis

ICD-9 Diagnosis

ICD-9 Diagnosis

ICD-9 Diagnosis

ICD-9 Diagnosis

ICD-9 Diagnosis

ICD-9 Diagnosis

ICD-9 Diagnosis

continued on next page 
APPENDIX B Code Lists (continued)

\section{Category}

Serious infection

Serious infection

Serious infection

Serious infection

Serious infection

Serious infection

Serious infection

Serious infection

Serious infection

Serious infection

Serious infection

Serious infection

Serious infection

Serious infection

Serious infection

Serious infection

Serious infection

Serious infection

Serious infection

Serious infection

Serious infection

Serious infection

Serious infection

Serious infection

Serious infection

Serious infection

Serious infection

Serious infection

Serious infection

Serious infection

Serious infection

Serious infection

Serious infection

Serious infection

Serious infection

Serious infection

Serious infection

Serious infection

Serious infection

Serious infection

Serious infection

Serious infection

\section{Description}

Infectious arthritis

Bacterial infection

Bacterial infection

Bacterial infection

Bacterial infection

Bacterial infection

Bacterial infection

Bacterial infection

Bacterial infection

Bacterial infection

Bacterial infection

Bacterial infection

Bacterial infection

Bacterial infection

Bacterial infection

Bacterial infection

Bacterial infection

Bacterial infection

Bacterial infection

Bacterial infection

Bacterial infection

Bacterial infection

Bacterial infection

Bacterial infection

Bacterial infection

Bacterial infection

Bacterial infection

Bacterial infection

Bacterial infection

Bacterial infection

Bacterial infection

Bacterial infection

Bacterial infection

Bacterial infection

Bacterial infection

Bacterial infection

Bacterial infection

Bacterial infection

Bacterial infection

Bacterial infection

Bacterial infection

Bacterial infection

\begin{tabular}{|c|c|}
\hline Code & CodeType \\
\hline 730.99 & ICD-9 Diagnosis \\
\hline 020.0 & ICD-9 Diagnosis \\
\hline 020.8 & ICD-9 Diagnosis \\
\hline 020.9 & ICD-9 Diagnosis \\
\hline 021.8 & ICD-9 Diagnosis \\
\hline 021.9 & ICD-9 Diagnosis \\
\hline 022.8 & ICD-9 Diagnosis \\
\hline 022.9 & ICD-9 Diagnosis \\
\hline 023.0 & ICD-9 Diagnosis \\
\hline 023.1 & ICD-9 Diagnosis \\
\hline 023.2 & ICD-9 Diagnosis \\
\hline 023.3 & ICD-9 Diagnosis \\
\hline 023.8 & ICD-9 Diagnosis \\
\hline 023.9 & ICD-9 Diagnosis \\
\hline 024 & ICD-9 Diagnosis \\
\hline 025 & ICD-9 Diagnosis \\
\hline 026.0 & ICD-9 Diagnosis \\
\hline 026.9 & ICD-9 Diagnosis \\
\hline 027.0 & ICD-9 Diagnosis \\
\hline 027.1 & ICD-9 Diagnosis \\
\hline 027.2 & ICD-9 Diagnosis \\
\hline 027.8 & ICD-9 Diagnosis \\
\hline 027.9 & ICD-9 Diagnosis \\
\hline 030.0 & ICD-9 Diagnosis \\
\hline 030.1 & ICD-9 Diagnosis \\
\hline 030.2 & ICD-9 Diagnosis \\
\hline 030.3 & ICD-9 Diagnosis \\
\hline 030.8 & ICD-9 Diagnosis \\
\hline 030.9 & ICD-9 Diagnosis \\
\hline 031.2 & ICD-9 Diagnosis \\
\hline 031.8 & ICD-9 Diagnosis \\
\hline 031.9 & ICD-9 Diagnosis \\
\hline 032.89 & ICD-9 Diagnosis \\
\hline 032.9 & ICD-9 Diagnosis \\
\hline 033.0 & ICD-9 Diagnosis \\
\hline 033.1 & ICD-9 Diagnosis \\
\hline 033.8 & ICD-9 Diagnosis \\
\hline 033.9 & ICD-9 Diagnosis \\
\hline 034.1 & ICD-9 Diagnosis \\
\hline 036.3 & ICD-9 Diagnosis \\
\hline 036.81 & ICD-9 Diagnosis \\
\hline 036.89 & ICD-9 Diagnosis \\
\hline
\end{tabular}


APPENDIX B Code Lists (continued)

\section{Category}

Serious infection

Serious infection

Serious infection

Serious infection

Serious infection

Serious infection

Serious infection

Serious infection

Serious infection

Serious infection

Serious infection

Serious infection

Serious infection

Serious infection

Serious infection

Serious infection

Serious infection

Serious infection

Serious infection

Serious infection

Serious infection

Serious infection

Serious infection

Serious infection

Serious infection

Serious infection

Serious infection

Serious infection

Serious infection

Serious infection

Serious infection

Serious infection

Serious infection

Serious infection

Serious infection

Serious infection

Serious infection

Serious infection

Serious infection

Serious infection

Serious infection

Serious infection

\section{Description}

Bacterial infection

Bacterial infection

Bacterial infection

Bacterial infection

Bacterial infection

Bacterial infection

Bacterial infection

Bacterial infection

Bacterial infection

Bacterial infection

Bacterial infection

Bacterial infection

Bacterial infection

Bacterial infection

Bacterial infection

Bacterial infection

Bacterial infection

Bacterial infection

Bacterial infection

Bacterial infection

Bacterial infection

Bacterial infection

Bacterial infection

Bacterial infection

Bacterial infection

Bacterial infection

Bacterial infection

Bacterial infection

Bacterial infection

Bacterial infection

Bacterial infection

Bacterial infection

Bacterial infection

Bacterial infection

Bacterial infection

Bacterial infection

Bacterial infection

Bacterial infection

Bacterial infection

Bacterial infection

Bacterial infection

Bacterial infection

\section{Code}

036.9

037

039.2

039.3

039.4

039.8

039.9

040.0

040.1

040.2

040.3

040.42

040.81

040.82

040.89

041.0

041.00

041.01

041.02

041.03

041.04

041.05

041.09

041.1

041.10

041.11

041.12

041.19

041.2

041.3

041.4

041.41

041.49

041.5

041.6

041.7

041.8

041.81

041.82

041.83

041.84

041.85
CodeType

ICD-9 Diagnosis

ICD-9 Diagnosis

ICD-9 Diagnosis

ICD-9 Diagnosis

ICD-9 Diagnosis

ICD-9 Diagnosis

ICD-9 Diagnosis

ICD-9 Diagnosis

ICD-9 Diagnosis

ICD-9 Diagnosis

ICD-9 Diagnosis

ICD-9 Diagnosis

ICD-9 Diagnosis

ICD-9 Diagnosis

ICD-9 Diagnosis

ICD-9 Diagnosis

ICD-9 Diagnosis

ICD-9 Diagnosis

ICD-9 Diagnosis

ICD-9 Diagnosis

ICD-9 Diagnosis

ICD-9 Diagnosis

ICD-9 Diagnosis

ICD-9 Diagnosis

ICD-9 Diagnosis

ICD-9 Diagnosis

ICD-9 Diagnosis

ICD-9 Diagnosis

ICD-9 Diagnosis

ICD-9 Diagnosis

ICD-9 Diagnosis

ICD-9 Diagnosis

ICD-9 Diagnosis

ICD-9 Diagnosis

ICD-9 Diagnosis

ICD-9 Diagnosis

ICD-9 Diagnosis

ICD-9 Diagnosis

ICD-9 Diagnosis

ICD-9 Diagnosis

ICD-9 Diagnosis

ICD-9 Diagnosis

continued on next page 
APPENDIX B Code Lists (continued)

\section{Category}

Serious infection

Serious infection

Serious infection

Serious infection

Serious infection

Serious infection

Serious infection

Serious infection

Serious infection

Serious infection

Serious infection

Serious infection

Serious infection

Serious infection

Serious infection

Serious infection

Serious infection

Serious infection

Serious infection

Serious infection

Serious infection

Serious infection

Serious infection

Serious infection

Serious infection

Serious infection

Serious infection

Serious infection

Serious infection

Serious infection

Serious infection

Serious infection

Serious infection

Serious infection

Serious infection

Serious infection

Serious infection

Serious infection

Serious infection

Serious infection

Serious infection

Serious infection

\section{Description}

Bacterial infection

Bacterial infection

Bacterial infection

Bacterial infection

Bacterial infection

Bacterial infection

Bacterial infection

Bacterial infection

Bacterial infection

Bacterial infection

Bacterial infection

Bacterial infection

Bacterial infection

Bacterial infection

Bacterial infection

Bacterial infection

Bacterial infection

Bacterial infection

Bacterial infection

Bacterial infection

Bacterial infection

Bacterial infection

Bacterial infection

Bacterial infection

Bacterial infection

Bacterial infection

Bronchitis

Bronchitis

Bronchitis

Bronchitis

Carditis

Carditis

Carditis

Carditis

Carditis

Carditis

Carditis

Carditis

Carditis

Carditis

Carditis

Carditis

\section{Code}

041.86

041.89

041.9

078.3

390

392.9

795.3

795.31

795.39

041.42

041.43

V09.0

V09.1

V09.2

V09.3

V09.4

V09.50

V09.51

V09.6

V09.70

V09.71

V09.80

V09.81

V09.90

v09.91

V12.04

466.0

466.1

466.11

466.19

032.82

036.40

036.41

036.42

036.43

074.20

074.21

074.22

074.23

112.81

115.03

115.04
CodeType

ICD-9 Diagnosis

ICD-9 Diagnosis

ICD-9 Diagnosis

ICD-9 Diagnosis

ICD-9 Diagnosis

ICD-9 Diagnosis

ICD-9 Diagnosis

ICD-9 Diagnosis

ICD-9 Diagnosis

ICD-9 Diagnosis

ICD-9 Diagnosis

ICD-9 Diagnosis

ICD-9 Diagnosis

ICD-9 Diagnosis

ICD-9 Diagnosis

ICD-9 Diagnosis

ICD-9 Diagnosis

ICD-9 Diagnosis

ICD-9 Diagnosis

ICD-9 Diagnosis

ICD-9 Diagnosis

ICD-9 Diagnosis

ICD-9 Diagnosis

ICD-9 Diagnosis

ICD-9 Diagnosis

ICD-9 Diagnosis

ICD-9 Diagnosis

ICD-9 Diagnosis

ICD-9 Diagnosis

ICD-9 Diagnosis

ICD-9 Diagnosis

ICD-9 Diagnosis

ICD-9 Diagnosis

ICD-9 Diagnosis

ICD-9 Diagnosis

ICD-9 Diagnosis

ICD-9 Diagnosis

ICD-9 Diagnosis

ICD-9 Diagnosis

ICD-9 Diagnosis

ICD-9 Diagnosis

ICD-9 Diagnosis

continued on next page 
APPENDIX B Code Lists (continued)

\section{Category}

Serious infection

Serious infection

Serious infection

Serious infection

Serious infection

Serious infection

Serious infection

Serious infection

Serious infection

Serious infection

Serious infection

Serious infection

Serious infection

Serious infection

Serious infection

Serious infection

Serious infection

Serious infection

Serious infection

Serious infection

Serious infection

Serious infection

Serious infection

Serious infection

Serious infection

Serious infection

Serious infection

Serious infection

Serious infection

Serious infection

Serious infection

Serious infection

Serious infection

Serious infection

Serious infection

Serious infection

Serious infection

Serious infection

Serious infection

Serious infection

Serious infection

Serious infection

\section{Description}

Carditis

Carditis

Carditis

Carditis

Carditis

Carditis

Carditis

Carditis

Carditis

Carditis

Carditis

Carditis

Carditis

Carditis

Carditis

Carditis

Carditis

Carditis

Carditis

Carditis

Carditis

Carditis

Carditis

Carditis

Carditis

Carditis

Carditis

Carditis

Carditis

Carditis

Carditis

Carditis

Carditis

Carditis

Carditis

Carditis

Carditis

Carditis

Carditis

Carditis

Carditis

Carditis

\section{Code}

115.13

115.14

115.93

115.94

130.3

391.0

391.1

391.2

391.8

391.9

392.0

393

398.0

398.90

398.99

420.0

420.90

420.91

420.99

421.0

421.1

421.9

422.0

422.90

422.91

422.92

422.93

422.99

423.0

423.1

423.2

423.3

423.8

423.9

425.0

425.1

425.11

425.18

425.2

425.3

425.4

425.7
CodeType

ICD-9 Diagnosis

ICD-9 Diagnosis

ICD-9 Diagnosis

ICD-9 Diagnosis

ICD-9 Diagnosis

ICD-9 Diagnosis

ICD-9 Diagnosis

ICD-9 Diagnosis

ICD-9 Diagnosis

ICD-9 Diagnosis

ICD-9 Diagnosis

ICD-9 Diagnosis

ICD-9 Diagnosis

ICD-9 Diagnosis

ICD-9 Diagnosis

ICD-9 Diagnosis

ICD-9 Diagnosis

ICD-9 Diagnosis

ICD-9 Diagnosis

ICD-9 Diagnosis

ICD-9 Diagnosis

ICD-9 Diagnosis

ICD-9 Diagnosis

ICD-9 Diagnosis

ICD-9 Diagnosis

ICD-9 Diagnosis

ICD-9 Diagnosis

ICD-9 Diagnosis

ICD-9 Diagnosis

ICD-9 Diagnosis

ICD-9 Diagnosis

ICD-9 Diagnosis

ICD-9 Diagnosis

ICD-9 Diagnosis

ICD-9 Diagnosis

ICD-9 Diagnosis

ICD-9 Diagnosis

ICD-9 Diagnosis

ICD-9 Diagnosis

ICD-9 Diagnosis

ICD-9 Diagnosis

ICD-9 Diagnosis

continued on next page 
APPENDIX B Code Lists (continued)

\section{Category}

Serious infection

Serious infection

Serious infection

Serious infection

Serious infection

Serious infection

Serious infection

Serious infection

Serious infection

Serious infection

Serious infection

Serious infection

Serious infection

Serious infection

Serious infection

Serious infection

Serious infection

Serious infection

Serious infection

Serious infection

Serious infection

Serious infection

Serious infection

Serious infection

Serious infection

Serious infection

Serious infection

Serious infection

Serious infection

Serious infection

Serious infection

Serious infection

Serious infection

Serious infection

Serious infection

Serious infection

Serious infection

Serious infection

Serious infection

Serious infection

Serious infection

Serious infection

\section{Description}

Carditis

Carditis

Carditis

Infection other CNS

Infection other CNS

Infection other CNS

Infection other CNS

Infection other CNS

Infection other CNS

Infection other CNS

Infection other CNS

Infection other CNS

Infection other CNS

Infection other CNS

Infection other CNS

Infection other CNS

Infection other CNS

Infection other CNS

Infection other CNS

Infection other CNS

Infection other CNS

Infection other CNS

Infection other CNS

Infection other CNS

Infection other CNS

Infection other CNS

Infection other CNS

Infection other CNS

Infection other CNS

Infection other CNS

Infection other CNS

Infection other CNS

Infection other CNS

Infection other CNS

Infection other CNS

Infection other CNS

Infection other complications

Infection other complications

Infection other complications

Infection other complications

Infection other complications

Infection other complications

\section{Code}

425.8

425.9

429.0

045.00

045.01

045.02

045.03

045.10

045.11

045.12

045.13

045.20

045.21

045.22

045.23

045.90

045.91

045.92

045.93

046.0

046.1

046.11

046.19

046.3

046.71

046.72

046.79

046.8

046.9

048

138

324.0

324.1

324.9

326

V12.02

279.50

279.51

279.52

279.53

414.02

414.03

\section{CodeType}

ICD-9 Diagnosis

ICD-9 Diagnosis

ICD-9 Diagnosis

ICD-9 Diagnosis

ICD-9 Diagnosis

ICD-9 Diagnosis

ICD-9 Diagnosis

ICD-9 Diagnosis

ICD-9 Diagnosis

ICD-9 Diagnosis

ICD-9 Diagnosis

ICD-9 Diagnosis

ICD-9 Diagnosis

ICD-9 Diagnosis

ICD-9 Diagnosis

ICD-9 Diagnosis

ICD-9 Diagnosis

ICD-9 Diagnosis

ICD-9 Diagnosis

ICD-9 Diagnosis

ICD-9 Diagnosis

ICD-9 Diagnosis

ICD-9 Diagnosis

ICD-9 Diagnosis

ICD-9 Diagnosis

ICD-9 Diagnosis

ICD-9 Diagnosis

ICD-9 Diagnosis

ICD-9 Diagnosis

ICD-9 Diagnosis

ICD-9 Diagnosis

ICD-9 Diagnosis

ICD-9 Diagnosis

ICD-9 Diagnosis

ICD-9 Diagnosis

ICD-9 Diagnosis

ICD-9 Diagnosis

ICD-9 Diagnosis

ICD-9 Diagnosis

ICD-9 Diagnosis

ICD-9 Diagnosis

ICD-9 Diagnosis

continued on next page 
APPENDIX B Code Lists (continued)

\section{Category}

Serious infection

Serious infection

Serious infection

Serious infection

Serious infection

Serious infection

Serious infection

Serious infection

Serious infection

Serious infection

Serious infection

Serious infection

Serious infection

Serious infection

Serious infection

Serious infection

Serious infection

Serious infection

Serious infection

Serious infection

Serious infection

Serious infection

Serious infection

Serious infection

Serious infection

Serious infection

Serious infection

Serious infection

Serious infection

Serious infection

Serious infection

Serious infection

Serious infection

Serious infection

Serious infection

Serious infection

Serious infection

Serious infection

Serious infection

Serious infection

Serious infection

Serious infection

\section{Description}

Infection other complications

Infection other complications

Infection other complications

Infection other complications

Infection other complications

Infection other complications

Infection other complications

Infection other complications

Infection other complications

Infection other complications

Infection other complications

Infection other complications

Infection other complications

Infection other complications

Infection other complications

Infection other complications

Infection other complications

Infection other complications

Infection other complications

Infection other complications

Infection other complications

Infection other complications

Infection other complications

Infection other complications

Infection other complications

Infection other complications

Infection other complications

Infection other complications

Infection other complications

Infection other complications

Infection other complications

Infection other complications

Infection other complications

Infection other complications

Infection other complications

Infection other complications

Infection other complications

Infection other complications

Infection other complications

Infection other complications

Infection other complications

Infection other complications

\section{Code}

414.04

414.05

414.07

440.30

440.31

440.32

569.60

569.61

569.69

596.82

596.83

629.31

629.32

996.00

996.01

996.02

996.03

996.04

996.09

996.1

996.2

996.30

996.31

996.32

996.39

996.4

996.40

996.41

996.42

996.43

996.44

996.45

996.46

996.47

996.49

996.51

996.52

996.53

996.54

996.55

996.56

996.57
CodeType

ICD-9 Diagnosis

ICD-9 Diagnosis

ICD-9 Diagnosis

ICD-9 Diagnosis

ICD-9 Diagnosis

ICD-9 Diagnosis

ICD-9 Diagnosis

ICD-9 Diagnosis

ICD-9 Diagnosis

ICD-9 Diagnosis

ICD-9 Diagnosis

ICD-9 Diagnosis

ICD-9 Diagnosis

ICD-9 Diagnosis

ICD-9 Diagnosis

ICD-9 Diagnosis

ICD-9 Diagnosis

ICD-9 Diagnosis

ICD-9 Diagnosis

ICD-9 Diagnosis

ICD-9 Diagnosis

ICD-9 Diagnosis

ICD-9 Diagnosis

ICD-9 Diagnosis

ICD-9 Diagnosis

ICD-9 Diagnosis

ICD-9 Diagnosis

ICD-9 Diagnosis

ICD-9 Diagnosis

ICD-9 Diagnosis

ICD-9 Diagnosis

ICD-9 Diagnosis

ICD-9 Diagnosis

ICD-9 Diagnosis

ICD-9 Diagnosis

ICD-9 Diagnosis

ICD-9 Diagnosis

ICD-9 Diagnosis

ICD-9 Diagnosis

ICD-9 Diagnosis

ICD-9 Diagnosis

ICD-9 Diagnosis

continued on next page 
APPENDIX B Code Lists (continued)

\section{Category}

Serious infection

Serious infection

Serious infection

Serious infection

Serious infection

Serious infection

Serious infection

Serious infection

Serious infection

Serious infection

Serious infection

Serious infection

Serious infection

Serious infection

Serious infection

Serious infection

Serious infection

Serious infection

Serious infection

Serious infection

Serious infection

Serious infection

Serious infection

Serious infection

Serious infection

Serious infection

Serious infection

Serious infection

Serious infection

Serious infection

Serious infection

Serious infection

Serious infection

Serious infection

Serious infection

Serious infection

Serious infection

Serious infection

Serious infection

Serious infection

Serious infection

Serious infection

\section{Description}

Infection other complications

Infection other complications

Infection other complications

Infection other complications

Infection other complications

Infection other complications

Infection other complications

Infection other complications

Infection other complications

Infection other complications

Infection other complications

Infection other complications

Infection other complications

Infection other complications

Infection other complications

Infection other complications

Infection other complications

Infection other complications

Infection other complications

Infection other complications

Infection other complications

Infection other complications

Infection other complications

Infection other complications

Infection other complications

Infection other complications

Infection other complications

Infection other complications

Infection other complications

Infection other complications

Infection other complications

Infection other complications

Infection other complications

Infection other complications

Infection other complications

Infection other complications

Infection other complications

Infection other complications

Infection other complications

Infection other complications

Infection other complications

Infection other complications

\section{Code}

996.59

996.6

996.60

996.61

996.62

996.63

996.64

996.65

996.66

996.67

996.68

996.69

996.7

996.70

996.71

996.72

996.73

996.74

996.75

996.76

996.77

996.78

996.79

996.80

996.81

996.82

996.83

996.84

996.85

996.86

996.87

996.88

996.89

996.90

996.91

996.92

996.93

996.94

996.95

996.96

996.99

999.31

\section{CodeType}

ICD-9 Diagnosis

ICD-9 Diagnosis

ICD-9 Diagnosis

ICD-9 Diagnosis

ICD-9 Diagnosis

ICD-9 Diagnosis

ICD-9 Diagnosis

ICD-9 Diagnosis

ICD-9 Diagnosis

ICD-9 Diagnosis

ICD-9 Diagnosis

ICD-9 Diagnosis

ICD-9 Diagnosis

ICD-9 Diagnosis

ICD-9 Diagnosis

ICD-9 Diagnosis

ICD-9 Diagnosis

ICD-9 Diagnosis

ICD-9 Diagnosis

ICD-9 Diagnosis

ICD-9 Diagnosis

ICD-9 Diagnosis

ICD-9 Diagnosis

ICD-9 Diagnosis

ICD-9 Diagnosis

ICD-9 Diagnosis

ICD-9 Diagnosis

ICD-9 Diagnosis

ICD-9 Diagnosis

ICD-9 Diagnosis

ICD-9 Diagnosis

ICD-9 Diagnosis

ICD-9 Diagnosis

ICD-9 Diagnosis

ICD-9 Diagnosis

ICD-9 Diagnosis

ICD-9 Diagnosis

ICD-9 Diagnosis

ICD-9 Diagnosis

ICD-9 Diagnosis

ICD-9 Diagnosis

ICD-9 Diagnosis

continued on next page 
APPENDIX B Code Lists (continued)

\section{Category}

Serious infection

Serious infection

Serious infection

Serious infection

Serious infection

Serious infection

Serious infection

Serious infection

Serious infection

Serious infection

Serious infection

Serious infection

Serious infection

Serious infection

Serious infection

Serious infection

Serious infection

Serious infection

Serious infection

Serious infection

Serious infection

Serious infection

Serious infection

Serious infection

Serious infection

Serious infection

Serious infection

Serious infection

Serious infection

Serious infection

Serious infection

Serious infection

Serious infection

Serious infection

Serious infection

Serious infection

Serious infection

Serious infection

Serious infection

Serious infection

Serious infection

Serious infection

\section{Description}

Infection other complications

Infection other complications

COPD

COPD

COPD

COPD

COPD

COPD

COPD

COPD

COPD

COPD

COPD

COPD

COPD

COPD

COPD

Diverticulitis

Diverticulitis

Diverticulitis

Diverticulitis

Diverticulitis

Diverticulitis

Diverticulitis

Diverticulitis

Encephalitis

Encephalitis

Encephalitis

Encephalitis

Encephalitis

Encephalitis

Encephalitis

Encephalitis

Encephalitis

Encephalitis

Encephalitis

Encephalitis

Encephalitis

Encephalitis

Encephalitis

Encephalitis

Encephalitis
Code
999.32
999.33
490
491.0
491.1
491.2
491.20
491.21
491.22
491.8
491.9
492.0
492.8
494
494.0
494.1
496
562.00
562.01
562.02
562.03
562.10
562.11
562.12
562.13
036.1
046.2
049.8
049.9
052.0
054.3
055.0
056.01
058.21
058.29
062.0
062.1
062.2
062.3
062.4
062.5
062.8

\section{CodeType}
ICD-9 Diagnosis
ICD-9 Diagnosis
ICD-9 Diagnosis
ICD-9 Diagnosis
ICD-9 Diagnosis
ICD-9 Diagnosis
ICD-9 Diagnosis
ICD-9 Diagnosis
ICD-9 Diagnosis
ICD-9 Diagnosis
ICD-9 Diagnosis
ICD-9 Diagnosis
ICD-9 Diagnosis
ICD-9 Diagnosis
ICD-9 Diagnosis
ICD-9 Diagnosis
ICD-9 Diagnosis
ICD-9 Diagnosis
ICD-9 Diagnosis
ICD-9 Diagnosis
ICD-9 Diagnosis
ICD-9 Diagnosis
ICD-9 Diagnosis
ICD-9 Diagnosis
ICD-9 Diagnosis
ICD-9 Diagnosis
ICD-9 Diagnosis
ICD-9 Diagnosis
ICD-9 Diagnosis
ICD-9 Diagnosis
ICD-9 Diagnosis
ICD-9 Diagnosis
ICD-9 Diagnosis
ICD-9 Diagnosis
ICD-9 Diagnosis
ICD-9 Diagnosis
ICD-9 Diagnosis
ICD-9 Diagnosis
ICD-9 Diagnosis
ICD-9 Diagnosis
ICD-9 Diagnosis
ICD-9 Diagnosis

continued on next page 
APPENDIX B Code Lists (continued)

\section{Category}

Serious infection

Serious infection

Serious infection

Serious infection

Serious infection

Serious infection

Serious infection

Serious infection

Serious infection

Serious infection

Serious infection

Serious infection

Serious infection

Serious infection

Serious infection

Serious infection

Serious infection

Serious infection

Serious infection

Serious infection

Serious infection

Serious infection

Serious infection

Serious infection

Serious infection

Serious infection

Serious infection

Serious infection

Serious infection

Serious infection

Serious infection

Serious infection

Serious infection

Serious infection

Serious infection

Serious infection

Serious infection

Serious infection

Serious infection

Serious infection

Serious infection

Serious infection

\section{Description}

Encephalitis

Encephalitis

Encephalitis

Encephalitis

Encephalitis

Encephalitis

Encephalitis

Encephalitis

Encephalitis

Encephalitis

Encephalitis

Encephalitis

Encephalitis

Encephalitis

Encephalitis

Encephalitis

Encephalitis

Encephalitis

Encephalitis

Encephalitis

Encephalitis

Encephalitis

Encephalitis

Encephalitis

Encephalitis

Encephalitis

Encephalitis

Encephalitis

Encephalitis

Encephalitis

Encephalitis

Encephalitis

Encephalitis

Encephalitis

Encephalitis

Encephalitis

Encephalitis

EYE

EYE

EYE

EYE

EYE

\begin{tabular}{|c|c|}
\hline Code & CodeType \\
\hline 062.9 & ICD-9 Diagnosis \\
\hline 063.0 & ICD-9 Diagnosis \\
\hline 063.1 & ICD-9 Diagnosis \\
\hline 063.2 & ICD-9 Diagnosis \\
\hline 063.8 & ICD-9 Diagnosis \\
\hline 063.9 & ICD-9 Diagnosis \\
\hline 064 & ICD-9 Diagnosis \\
\hline 066.2 & ICD-9 Diagnosis \\
\hline 066.41 & ICD-9 Diagnosis \\
\hline 072.2 & ICD-9 Diagnosis \\
\hline 130.0 & ICD-9 Diagnosis \\
\hline 139.0 & ICD-9 Diagnosis \\
\hline 323.0 & ICD-9 Diagnosis \\
\hline 323.01 & ICD-9 Diagnosis \\
\hline 323.02 & ICD-9 Diagnosis \\
\hline 323.1 & ICD-9 Diagnosis \\
\hline 323.2 & ICD-9 Diagnosis \\
\hline 323.4 & ICD-9 Diagnosis \\
\hline 323.41 & ICD-9 \\
\hline 323.42 & ICD-9 Diagnosis \\
\hline 323.5 & ICD-9 Diagnosis \\
\hline 323.51 & ICD-9 Diagnosis \\
\hline 323.52 & ICD-9 Diagnosis \\
\hline 323.6 & ICD-9 Diagnosis \\
\hline 323.61 & ICD-9 Diagnosis \\
\hline 323.62 & ICD-9 Diagnosis \\
\hline 323.63 & ICD-9 Diagnosis \\
\hline 323.7 & ICD-9 Diagnosis \\
\hline 323.71 & ICD-9 Diagnosis \\
\hline 323.72 & ICD-9 Diagnosis \\
\hline 323.8 & ICD-9 Diagnosis \\
\hline 323.81 & ICD-9 Diagnosis \\
\hline 323.82 & ICD-9 Diagnosis \\
\hline 323.9 & ICD-9 Diagnosis \\
\hline 341.20 & ICD-9 Diagnosis \\
\hline 341.21 & ICD-9 Diagnosis \\
\hline 341.22 & ICD-9 Diagnosis \\
\hline 021.3 & ICD-9 Diagnosis \\
\hline 032.81 & ICD-9 Diagnosis \\
\hline 053.20 & ICD-9 Diagnosis \\
\hline 053.21 & ICD-9 Diagnosis \\
\hline 053.22 & ICD-9 Diagnosis \\
\hline
\end{tabular}


APPENDIX B Code Lists (continued)

\section{Category}

Serious infection

Serious infection

Serious infection

Serious infection

Serious infection

Serious infection

Serious infection

Serious infection

Serious infection

Serious infection

Serious infection

Serious infection

Serious infection

Serious infection

Serious infection

Serious infection

Serious infection

Serious infection

Serious infection

Serious infection

Serious infection

Serious infection

Serious infection

Serious infection

Serious infection

Serious infection

Serious infection

Serious infection

Serious infection

Serious infection

Serious infection

Serious infection

Serious infection

Serious infection

Serious infection

Serious infection

Serious infection

Serious infection

Serious infection

Serious infection

Serious infection

Serious infection

\section{Description}

EYE

EYE

EYE

EYE

EYE

EYE

EYE

EYE

EYE

EYE

EYE

EYE

EYE

EYE

EYE

EYE

EYE

EYE

EYE

EYE

EYE

EYE

EYE

EYE

EYE

EYE

EYE

EYE

EYE

EYE

EYE

EYE

EYE

EYE

EYE

EYE

EYE

EYE

EYE

EYE

EYE

EYE

\section{Code}

053.29

054.40

054.41

054.42

054.43

054.44

054.49

055.71

076.0

076.1

076.9

077.0

077.1

077.2

077.3

077.4

077.8

077.9

077.98

077.99

115.02

115.12

115.92

130.1

130.2

139.1

360.00

360.01

360.02

360.03

360.04

360.11

360.12

360.13

360.14

360.19

363.00

363.01

363.03

363.04

363.05

363.06
CodeType

ICD-9 Diagnosis

ICD-9 Diagnosis

ICD-9 Diagnosis

ICD-9 Diagnosis

ICD-9 Diagnosis

ICD-9 Diagnosis

ICD-9 Diagnosis

ICD-9 Diagnosis

ICD-9 Diagnosis

ICD-9 Diagnosis

ICD-9 Diagnosis

ICD-9 Diagnosis

ICD-9 Diagnosis

ICD-9 Diagnosis

ICD-9 Diagnosis

ICD-9 Diagnosis

ICD-9 Diagnosis

ICD-9 Diagnosis

ICD-9 Diagnosis

ICD-9 Diagnosis

ICD-9 Diagnosis

ICD-9 Diagnosis

ICD-9 Diagnosis

ICD-9 Diagnosis

ICD-9 Diagnosis

ICD-9 Diagnosis

ICD-9 Diagnosis

ICD-9 Diagnosis

ICD-9 Diagnosis

ICD-9 Diagnosis

ICD-9 Diagnosis

ICD-9 Diagnosis

ICD-9 Diagnosis

ICD-9 Diagnosis

ICD-9 Diagnosis

ICD-9 Diagnosis

ICD-9 Diagnosis

ICD-9 Diagnosis

ICD-9 Diagnosis

ICD-9 Diagnosis

ICD-9 Diagnosis

ICD-9 Diagnosis

continued on next page 
APPENDIX B Code Lists (continued)

\section{Category}

Serious infection

Serious infection

Serious infection

Serious infection

Serious infection

Serious infection

Serious infection

Serious infection

Serious infection

Serious infection

Serious infection

Serious infection

Serious infection

Serious infection

Serious infection

Serious infection

Serious infection

Serious infection

Serious infection

Serious infection

Serious infection

Serious infection

Serious infection

Serious infection

Serious infection

Serious infection

Serious infection

Serious infection

Serious infection

Serious infection

Serious infection

Serious infection

Serious infection

Serious infection

Serious infection

Serious infection

Serious infection

Serious infection

Serious infection

Serious infection

Serious infection

Serious infection

\section{Description}

EYE

EYE

EYE

EYE

EYE

EYE

EYE

EYE

EYE

EYE

EYE

EYE

EYE

EYE

EYE

EYE

EYE

EYE

EYE

EYE

EYE

EYE

EYE

EYE

EYE

EYE

EYE

EYE

EYE

EYE

EYE

EYE

EYE

EYE

EYE

EYE

EYE

EYE

EYE

EYE

EYE

EYE

\section{Code}

363.07

363.08

363.10

363.11

363.12

363.13

363.14

363.15

363.20

363.21

363.22

364.00

364.01

364.02

364.03

364.04

364.05

364.10

364.11

364.21

364.22

364.23

364.24

364.3

370.20

370.21

370.22

370.23

370.24

370.31

370.32

370.33

370.34

370.35

370.40

370.44

370.49

370.50

370.52

370.54

370.55

370.59
CodeType

ICD-9 Diagnosis

ICD-9 Diagnosis

ICD-9 Diagnosis

ICD-9 Diagnosis

ICD-9 Diagnosis

ICD-9 Diagnosis

ICD-9 Diagnosis

ICD-9 Diagnosis

ICD-9 Diagnosis

ICD-9 Diagnosis

ICD-9 Diagnosis

ICD-9 Diagnosis

ICD-9 Diagnosis

ICD-9 Diagnosis

ICD-9 Diagnosis

ICD-9 Diagnosis

ICD-9 Diagnosis

ICD-9 Diagnosis

ICD-9 Diagnosis

ICD-9 Diagnosis

ICD-9 Diagnosis

ICD-9 Diagnosis

ICD-9 Diagnosis

ICD-9 Diagnosis

ICD-9 Diagnosis

ICD-9 Diagnosis

ICD-9 Diagnosis

ICD-9 Diagnosis

ICD-9 Diagnosis

ICD-9 Diagnosis

ICD-9 Diagnosis

ICD-9 Diagnosis

ICD-9 Diagnosis

ICD-9 Diagnosis

ICD-9 Diagnosis

ICD-9 Diagnosis

ICD-9 Diagnosis

ICD-9 Diagnosis

ICD-9 Diagnosis

ICD-9 Diagnosis

ICD-9 Diagnosis

ICD-9 Diagnosis

continued on next page 
APPENDIX B Code Lists (continued)

\section{Category}

Serious infection

Serious infection

Serious infection

Serious infection

Serious infection

Serious infection

Serious infection

Serious infection

Serious infection

Serious infection

Serious infection

Serious infection

Serious infection

Serious infection

Serious infection

Serious infection

Serious infection

Serious infection

Serious infection

Serious infection

Serious infection

Serious infection

Serious infection

Serious infection

Serious infection

Serious infection

Serious infection

Serious infection

Serious infection

Serious infection

Serious infection

Serious infection

Serious infection

Serious infection

Serious infection

Serious infection

Serious infection

Serious infection

Serious infection

Serious infection

Serious infection

Serious infection

\section{Description}

EYE

EYE

EYE

EYE

EYE

EYE

EYE

EYE

EYE

EYE

EYE

EYE

EYE

EYE

EYE

EYE

EYE

EYE

EYE

EYE

EYE

EYE

EYE

EYE

EYE

EYE

EYE

EYE

EYE

EYE

EYE

EYE

EYE

EYE

EYE

EYE

EYE

EYE

EYE

EYE

EYE

EYE

\section{Code}

370.8

370.9

372.00

372.01

372.02

372.03

372.04

372.05

372.06

372.10

372.11

372.12

372.13

372.14

372.15

372.20

372.21

372.22

372.30

372.31

372.33

372.39

373.00

373.01

373.02

373.11

373.12

373.13

373.31

373.32

373.33

373.34

373.4

373.5

373.6

373.8

373.9

375.00

375.01

375.02

375.03

375.30
CodeType

ICD-9 Diagnosis

ICD-9 Diagnosis

ICD-9 Diagnosis

ICD-9 Diagnosis

ICD-9 Diagnosis

ICD-9 Diagnosis

ICD-9 Diagnosis

ICD-9 Diagnosis

ICD-9 Diagnosis

ICD-9 Diagnosis

ICD-9 Diagnosis

ICD-9 Diagnosis

ICD-9 Diagnosis

ICD-9 Diagnosis

ICD-9 Diagnosis

ICD-9 Diagnosis

ICD-9 Diagnosis

ICD-9 Diagnosis

ICD-9 Diagnosis

ICD-9 Diagnosis

ICD-9 Diagnosis

ICD-9 Diagnosis

ICD-9 Diagnosis

ICD-9 Diagnosis

ICD-9 Diagnosis

ICD-9 Diagnosis

ICD-9 Diagnosis

ICD-9 Diagnosis

ICD-9 Diagnosis

ICD-9 Diagnosis

ICD-9 Diagnosis

ICD-9 Diagnosis

ICD-9 Diagnosis

ICD-9 Diagnosis

ICD-9 Diagnosis

ICD-9 Diagnosis

ICD-9 Diagnosis

ICD-9 Diagnosis

ICD-9 Diagnosis

ICD-9 Diagnosis

ICD-9 Diagnosis

ICD-9 Diagnosis

continued on next page 
APPENDIX B Code Lists (continued)

\section{Category}

Serious infection

Serious infection

Serious infection

Serious infection

Serious infection

Serious infection

Serious infection

Serious infection

Serious infection

Serious infection

Serious infection

Serious infection

Serious infection

Serious infection

Serious infection

Serious infection

Serious infection

Serious infection

Serious infection

Serious infection

Serious infection

Serious infection

Serious infection

Serious infection

Serious infection

Serious infection

Serious infection

Serious infection

Serious infection

Serious infection

Serious infection

Serious infection

Serious infection

Serious infection

Serious infection

Serious infection

Serious infection

Serious infection

Serious infection

Serious infection

Serious infection

Serious infection

\section{Description}

EYE

EYE

EYE

EYE

EYE

EYE

EYE

EYE

EYE

EYE

EYE

EYE

EYE

EYE

EYE

EYE

EYE

EYE

EYE

EYE

EYE

EYE

EYE

EYE

EYE

EYE

EYE

EYE

EYE

EYE

EYE

EYE

EYE

EYE

Gangrene

Gangrene

Hepatitis

Hepatitis

Hepatitis

Hepatitis

Hepatitis

Hepatitis

\section{Code}

375.31

375.32

375.33

375.41

375.42

375.43

376.00

376.01

376.02

376.03

376.04

376.10

376.11

376.12

376.13

377.30

377.31

377.32

377.33

377.34

377.39

379.00

379.01

379.02

379.03

379.04

379.05

379.06

379.07

379.09

379.60

379.61

379.62

379.63

440.24

785.4

070.0

070.1

070.2

070.20

070.21

070.22
CodeType

ICD-9 Diagnosis

ICD-9 Diagnosis

ICD-9 Diagnosis

ICD-9 Diagnosis

ICD-9 Diagnosis

ICD-9 Diagnosis

ICD-9 Diagnosis

ICD-9 Diagnosis

ICD-9 Diagnosis

ICD-9 Diagnosis

ICD-9 Diagnosis

ICD-9 Diagnosis

ICD-9 Diagnosis

ICD-9 Diagnosis

ICD-9 Diagnosis

ICD-9 Diagnosis

ICD-9 Diagnosis

ICD-9 Diagnosis

ICD-9 Diagnosis

ICD-9 Diagnosis

ICD-9 Diagnosis

ICD-9 Diagnosis

ICD-9 Diagnosis

ICD-9 Diagnosis

ICD-9 Diagnosis

ICD-9 Diagnosis

ICD-9 Diagnosis

ICD-9 Diagnosis

ICD-9 Diagnosis

ICD-9 Diagnosis

ICD-9 Diagnosis

ICD-9 Diagnosis

ICD-9 Diagnosis

ICD-9 Diagnosis

ICD-9 Diagnosis

ICD-9 Diagnosis

ICD-9 Diagnosis

ICD-9 Diagnosis

ICD-9 Diagnosis

ICD-9 Diagnosis

ICD-9 Diagnosis

ICD-9 Diagnosis

continued on next page 
APPENDIX B Code Lists (continued)

\section{Category}

Serious infection

Serious infection

Serious infection

Serious infection

Serious infection

Serious infection

Serious infection

Serious infection

Serious infection

Serious infection

Serious infection

Serious infection

Serious infection

Serious infection

Serious infection

Serious infection

Serious infection

Serious infection

Serious infection

Serious infection

Serious infection

Serious infection

Serious infection

Serious infection

Serious infection

Serious infection

Serious infection

Serious infection

Serious infection

Serious infection

Serious infection

Serious infection

Serious infection

Serious infection

Serious infection

Serious infection

Serious infection

Serious infection

Serious infection

Serious infection

Serious infection

Serious infection

\section{Description}

Hepatitis

Hepatitis

Hepatitis

Hepatitis

Hepatitis

Hepatitis

Hepatitis

Hepatitis

Hepatitis

Hepatitis

Hepatitis

Hepatitis

Hepatitis

Hepatitis

Hepatitis

Hepatitis

Hepatitis

Hepatitis

Hepatitis

Hepatitis

Hepatitis

Hepatitis

Hepatitis

Hepatitis

Hepatitis

Hepatitis

Hepatitis

Hepatitis

Hepatitis

Hepatitis

Hepatitis

Hepatitis

Hepatitis

Hepatitis

Hepatitis

Hepatitis

HIV

HIV

HIV

HIV

HIV

HIV

\section{Code}

070.23

070.3

070.30

070.31

070.32

070.33

070.4

070.41

070.41

070.42

070.43

070.44

070.44

070.49

070.5

070.51

070.51

070.52

070.53

070.54

070.54

070.59

070.6

070.7

070.70

070.71

070.9

072.71

571.40

571.41

571.42

571.49

573.1

573.2

573.3

V02.62

042

042.0

042.1

042.2

042.9

043.0
CodeType

ICD-9 Diagnosis

ICD-9 Diagnosis

ICD-9 Diagnosis

ICD-9 Diagnosis

ICD-9 Diagnosis

ICD-9 Diagnosis

ICD-9 Diagnosis

ICD-9 Diagnosis

ICD-9 Diagnosis

ICD-9 Diagnosis

ICD-9 Diagnosis

ICD-9 Diagnosis

ICD-9 Diagnosis

ICD-9 Diagnosis

ICD-9 Diagnosis

ICD-9 Diagnosis

ICD-9 Diagnosis

ICD-9 Diagnosis

ICD-9 Diagnosis

ICD-9 Diagnosis

ICD-9 Diagnosis

ICD-9 Diagnosis

ICD-9 Diagnosis

ICD-9 Diagnosis

ICD-9 Diagnosis

ICD-9 Diagnosis

ICD-9 Diagnosis

ICD-9 Diagnosis

ICD-9 Diagnosis

ICD-9 Diagnosis

ICD-9 Diagnosis

ICD-9 Diagnosis

ICD-9 Diagnosis

ICD-9 Diagnosis

ICD-9 Diagnosis

ICD-9 Diagnosis

ICD-9 Diagnosis

ICD-9 Diagnosis

ICD-9 Diagnosis

ICD-9 Diagnosis

ICD-9 Diagnosis

ICD-9 Diagnosis

continued on next page 
APPENDIX B Code Lists (continued)

\section{Category}

Serious infection

Serious infection

Serious infection

Serious infection

Serious infection

Serious infection

Serious infection

Serious infection

Serious infection

Serious infection

Serious infection

Serious infection

Serious infection

Serious infection

Serious infection

Serious infection

Serious infection

Serious infection

Serious infection

Serious infection

Serious infection

Serious infection

Serious infection

Serious infection

Serious infection

Serious infection

Serious infection

Serious infection

Serious infection

Serious infection

Serious infection

Serious infection

Serious infection

Serious infection

Serious infection

Serious infection

Serious infection

Serious infection

Serious infection

Serious infection

Serious infection

Serious infection

\section{Description}

HIV

HIV

HIV

HIV

HIV

HIV

HIV

HIV

HIV

HIV

HIV

HIV

INF

INF

INF

INF

INF

INF

INF

INF

INF

INF

INF

INF

INF

INF

INF

INF

INF

INF

Infection intestinal

Infection intestinal

Infection intestinal

Infection intestinal

Infection intestinal

Infection intestinal

Infection intestinal

Infection intestinal

Infection intestinal

Infection intestinal

Infection intestinal

Infection intestinal

\section{Code}

043.1

043.2

043.3

043.9

044.0

044.9

079.53

279.10

279.19

795.71

795.8

V08

487.0

487.1

487.8

488

488.0

488.01

488.02

488.09

488.1

488.11

488.12

488.19

488.81

488.82

488.89

V03.82

V04.81

V06.6

001.0

001.1

001.9

002.0

002.1

002.2

002.3

002.9

003.0

003.20

003.29

003.8
CodeType

ICD-9 Diagnosis

ICD-9 Diagnosis

ICD-9 Diagnosis

ICD-9 Diagnosis

ICD-9 Diagnosis

ICD-9 Diagnosis

ICD-9 Diagnosis

ICD-9 Diagnosis

ICD-9 Diagnosis

ICD-9 Diagnosis

ICD-9 Diagnosis

ICD-9 Diagnosis

ICD-9 Diagnosis

ICD-9 Diagnosis

ICD-9 Diagnosis

ICD-9 Diagnosis

ICD-9 Diagnosis

ICD-9 Diagnosis

ICD-9 Diagnosis

ICD-9 Diagnosis

ICD-9 Diagnosis

ICD-9 Diagnosis

ICD-9 Diagnosis

ICD-9 Diagnosis

ICD-9 Diagnosis

ICD-9 Diagnosis

ICD-9 Diagnosis

ICD-9 Diagnosis

ICD-9 Diagnosis

ICD-9 Diagnosis

ICD-9 Diagnosis

ICD-9 Diagnosis

ICD-9 Diagnosis

ICD-9 Diagnosis

ICD-9 Diagnosis

ICD-9 Diagnosis

ICD-9 Diagnosis

ICD-9 Diagnosis

ICD-9 Diagnosis

ICD-9 Diagnosis

ICD-9 Diagnosis

ICD-9 Diagnosis

continued on next page 
APPENDIX B Code Lists (continued)

\section{Category}

Serious infection

Serious infection

Serious infection

Serious infection

Serious infection

Serious infection

Serious infection

Serious infection

Serious infection

Serious infection

Serious infection

Serious infection

Serious infection

Serious infection

Serious infection

Serious infection

Serious infection

Serious infection

Serious infection

Serious infection

Serious infection

Serious infection

Serious infection

Serious infection

Serious infection

Serious infection

Serious infection

Serious infection

Serious infection

Serious infection

Serious infection

Serious infection

Serious infection

Serious infection

Serious infection

Serious infection

Serious infection

Serious infection

Serious infection

Serious infection

Serious infection

Serious infection

\section{Description}

Infection intestinal

Infection intestinal

Infection intestinal

Infection intestinal

Infection intestinal

Infection intestinal

Infection intestinal

Infection intestinal

Infection intestinal

Infection intestinal

Infection intestinal

Infection intestinal

Infection intestinal

Infection intestinal

Infection intestinal

Infection intestinal

Infection intestinal

Infection intestinal

Infection intestinal

Infection intestinal

Infection intestinal

Infection intestinal

Infection intestinal

Infection intestinal

Infection intestinal

Infection intestinal

Infection intestinal

Infection intestinal

Infection intestinal

Infection intestinal

Infection intestinal

Infection intestinal

Infection intestinal

Infection intestinal

Infection intestinal

Infection intestinal

Infection intestinal

Infection intestinal

Infection intestinal

Infection intestinal

Infection intestinal

Infection intestinal

\section{Code}

003.9

004.0

004.1

004.2

004.3

004.8

004.9

005.0

005.1

005.2

005.3

005.4

005.8

005.81

005.89

005.9

006.0

006.1

006.2

006.3

006.4

006.5

006.6

006.8

006.9

007.0

007.1

007.2

007.3

007.4

007.5

007.8

007.9

008.0

008.00

008.01

008.02

008.03

008.04

008.09

008.1

008.2
CodeType

ICD-9 Diagnosis

ICD-9 Diagnosis

ICD-9 Diagnosis

ICD-9 Diagnosis

ICD-9 Diagnosis

ICD-9 Diagnosis

ICD-9 Diagnosis

ICD-9 Diagnosis

ICD-9 Diagnosis

ICD-9 Diagnosis

ICD-9 Diagnosis

ICD-9 Diagnosis

ICD-9 Diagnosis

ICD-9 Diagnosis

ICD-9 Diagnosis

ICD-9 Diagnosis

ICD-9 Diagnosis

ICD-9 Diagnosis

ICD-9 Diagnosis

ICD-9 Diagnosis

ICD-9 Diagnosis

ICD-9 Diagnosis

ICD-9 Diagnosis

ICD-9 Diagnosis

ICD-9 Diagnosis

ICD-9 Diagnosis

ICD-9 Diagnosis

ICD-9 Diagnosis

ICD-9 Diagnosis

ICD-9 Diagnosis

ICD-9 Diagnosis

ICD-9 Diagnosis

ICD-9 Diagnosis

ICD-9 Diagnosis

ICD-9 Diagnosis

ICD-9 Diagnosis

ICD-9 Diagnosis

ICD-9 Diagnosis

ICD-9 Diagnosis

ICD-9 Diagnosis

ICD-9 Diagnosis

ICD-9 Diagnosis

continued on next page 
APPENDIX B Code Lists (continued)

\section{Category}

Serious infection

Serious infection

Serious infection

Serious infection

Serious infection

Serious infection

Serious infection

Serious infection

Serious infection

Serious infection

Serious infection

Serious infection

Serious infection

Serious infection

Serious infection

Serious infection

Serious infection

Serious infection

Serious infection

Serious infection

Serious infection

Serious infection

Serious infection

Serious infection

Serious infection

Serious infection

Serious infection

Serious infection

Serious infection

Serious infection

Serious infection

Serious infection

Serious infection

Serious infection

Serious infection

Serious infection

Serious infection

Serious infection

Serious infection

Serious infection

Serious infection

Serious infection

\section{Description}

Infection intestinal

Infection intestinal

Infection intestinal

Infection intestinal

Infection intestinal

Infection intestinal

Infection intestinal

Infection intestinal

Infection intestinal

Infection intestinal

Infection intestinal

Infection intestinal

Infection intestinal

Infection intestinal

Infection intestinal

Infection intestinal

Infection intestinal

Infection intestinal

Infection intestinal

Infection intestinal

Infection intestinal

Infection intestinal

Infection intestinal

Infection intestinal

Infection intestinal

Infection intestinal

Lymphadenitis

Lymphadenitis

Lymphadenitis

Lymphadenitis

Lymphadenitis

Meningitis

Meningitis

Meningitis

Meningitis

Meningitis

Meningitis

Meningitis

Meningitis

Meningitis

Meningitis

Meningitis

\section{Code}

008.3

008.41

008.42

008.43

008.44

008.45

008.46

008.47

008.49

008.5

008.6

008.61

008.62

008.63

008.64

008.65

008.66

008.67

008.69

008.8

009.0

009.1

009.2

009.3

021.1

022.2

289.1

289.2

289.3

683

785.6

003.21

036.0

047.0

047.1

047.8

047.9

049.0

049.1

053.0

054.72

072.1

\section{CodeType}

ICD-9 Diagnosis

ICD-9 Diagnosis

ICD-9 Diagnosis

ICD-9 Diagnosis

ICD-9 Diagnosis

ICD-9 Diagnosis

ICD-9 Diagnosis

ICD-9 Diagnosis

ICD-9 Diagnosis

ICD-9 Diagnosis

ICD-9 Diagnosis

ICD-9 Diagnosis

ICD-9 Diagnosis

ICD-9 Diagnosis

ICD-9 Diagnosis

ICD-9 Diagnosis

ICD-9 Diagnosis

ICD-9 Diagnosis

ICD-9 Diagnosis

ICD-9 Diagnosis

ICD-9 Diagnosis

ICD-9 Diagnosis

ICD-9 Diagnosis

ICD-9 Diagnosis

ICD-9 Diagnosis

ICD-9 Diagnosis

ICD-9 Diagnosis

ICD-9 Diagnosis

ICD-9 Diagnosis

ICD-9 Diagnosis

ICD-9 Diagnosis

ICD-9 Diagnosis

ICD-9 Diagnosis

ICD-9 Diagnosis

ICD-9 Diagnosis

ICD-9 Diagnosis

ICD-9 Diagnosis

ICD-9 Diagnosis

ICD-9 Diagnosis

ICD-9 Diagnosis

ICD-9 Diagnosis

ICD-9 Diagnosis

continued on next page 
APPENDIX B Code Lists (continued)

\section{Category}

Serious infection

Serious infection

Serious infection

Serious infection

Serious infection

Serious infection

Serious infection

Serious infection

Serious infection

Serious infection

Serious infection

Serious infection

Serious infection

Serious infection

Serious infection

Serious infection

Serious infection

Serious infection

Serious infection

Serious infection

Serious infection

Serious infection

Serious infection

Serious infection

Serious infection

Serious infection

Serious infection

Serious infection

Serious infection

Serious infection

Serious infection

Serious infection

Serious infection

Serious infection

Serious infection

Serious infection

Serious infection

Serious infection

Serious infection

Serious infection

Serious infection

Serious infection

\section{Description}

Meningitis

Meningitis

Meningitis

Meningitis

Meningitis

Meningitis

Meningitis

Meningitis

Meningitis

Meningitis

Meningitis

Meningitis

Meningitis

Meningitis

Meningitis

Meningitis

Meningitis

Meningitis

Meningitis

Meningitis

Meningitis

Meningitis

Meningitis

Meningitis

Meningitis

Meningitis

Mycoses

Mycoses

Mycoses

Mycoses

Mycoses

Mycoses

Mycoses

Mycoses

Mycoses

Mycoses

Mycoses

Mycoses

Mycoses

Mycoses

Mycoses

Mycoses

\begin{tabular}{|c|c|}
\hline Code & CodeType \\
\hline 100.81 & ICD-9 Diagnosis \\
\hline 112.83 & ICD-9 Diagnosis \\
\hline 114.2 & ICD-9 Diagnosis \\
\hline 115.01 & ICD-9 Diagnosis \\
\hline 115.11 & ICD-9 Diagnosis \\
\hline 115.91 & ICD-9 Diagnosis \\
\hline 320.0 & ICD-9 Diagnosis \\
\hline 320.1 & ICD-9 Diagnosis \\
\hline 320.2 & ICD-9 Diagnosis \\
\hline 320.3 & ICD-9 Diagnosis \\
\hline 320.7 & ICD-9 Diagnosis \\
\hline 320.8 & ICD-9 Diagnosis \\
\hline 320.81 & ICD-9 Diagnosis \\
\hline 320.82 & ICD-9 Diagnosis \\
\hline 320.89 & ICD-9 Diagnosis \\
\hline 320.9 & ICD-9 Diagnosis \\
\hline 321.0 & ICD-9 Diagnosis \\
\hline 321.1 & ICD-9 Diagnosis \\
\hline 321.2 & ICD-9 Diagnosis \\
\hline 321.3 & ICD-9 Diagnosis \\
\hline 321.4 & ICD-9 Diagnosis \\
\hline 321.8 & ICD-9 Diagnosis \\
\hline 322.0 & ICD-9 Diagnosis \\
\hline 322.1 & ICD-9 Diagnosis \\
\hline 322.2 & ICD-9 Diagnosis \\
\hline 322.9 & ICD-9 Diagnosis \\
\hline 110.0 & ICD-9 Diagnosis \\
\hline 110.1 & ICD-9 Diagnosis \\
\hline 110.2 & ICD-9 Diagnosis \\
\hline 110.3 & ICD-9 Diagnosis \\
\hline 110.4 & ICD-9 Diagnosis \\
\hline 110.5 & ICD-9 Diagnosis \\
\hline 110.6 & ICD-9 Diagnosis \\
\hline 110.8 & ICD-9 Diagnosis \\
\hline 110.9 & ICD-9 Diagnosis \\
\hline 111.0 & ICD-9 Diagnosis \\
\hline 111.1 & ICD-9 Diagnosis \\
\hline 111.2 & ICD-9 Diagnosis \\
\hline 111.3 & ICD-9 Diagnosis \\
\hline 111.8 & ICD-9 Diagnosis \\
\hline 111.9 & ICD-9 Diagr \\
\hline 112.0 & ICD-9 Diagnosi \\
\hline
\end{tabular}

continued on next page 
APPENDIX B Code Lists (continued)

\section{Category}

Serious infection

Serious infection

Serious infection

Serious infection

Serious infection

Serious infection

Serious infection

Serious infection

Serious infection

Serious infection

Serious infection

Serious infection

Serious infection

Serious infection

Serious infection

Serious infection

Serious infection

Serious infection

Serious infection

Serious infection

Serious infection

Serious infection

Serious infection

Serious infection

Serious infection

Serious infection

Serious infection

Serious infection

Serious infection

Serious infection

Serious infection

Serious infection

Serious infection

Serious infection

Serious infection

Serious infection

Serious infection

Serious infection

Serious infection

Serious infection

Serious infection

Serious infection

\section{Description}

Mycoses

Mycoses

Mycoses

Mycoses

Mycoses

Mycoses

Mycoses

Mycoses

Mycoses

Mycoses

Mycoses

Mycoses

Mycoses

Mycoses

Mycoses

Mycoses

Mycoses

Mycoses

Mycoses

Mycoses

Mycoses

Mycoses

Mycoses

Mycoses

Mycoses

Mycoses

Mycoses

Mycoses

Mycoses

Mycoses

Mycoses

Mycoses

Other

Other

Other

Other

Other

Other

Other

Other

Other

Other

\section{Code}

112.1

112.2

112.3

112.5

112.82

112.84

112.85

112.89

112.9

114.1

114.3

114.9

115.00

115.09

115.10

115.19

115.90

115.99

116.0

116.1

116.2

117.0

117.1

117.2

117.3

117.4

117.5

117.6

117.7

117.8

117.9

118

080

081.0

081.1

081.2

081.9

082.0

082.1

082.2

082.3

082.40
CodeType

ICD-9 Diagnosis

ICD-9 Diagnosis

ICD-9 Diagnosis

ICD-9 Diagnosis

ICD-9 Diagnosis

ICD-9 Diagnosis

ICD-9 Diagnosis

ICD-9 Diagnosis

ICD-9 Diagnosis

ICD-9 Diagnosis

ICD-9 Diagnosis

ICD-9 Diagnosis

ICD-9 Diagnosis

ICD-9 Diagnosis

ICD-9 Diagnosis

ICD-9 Diagnosis

ICD-9 Diagnosis

ICD-9 Diagnosis

ICD-9 Diagnosis

ICD-9 Diagnosis

ICD-9 Diagnosis

ICD-9 Diagnosis

ICD-9 Diagnosis

ICD-9 Diagnosis

ICD-9 Diagnosis

ICD-9 Diagnosis

ICD-9 Diagnosis

ICD-9 Diagnosis

ICD-9 Diagnosis

ICD-9 Diagnosis

ICD-9 Diagnosis

ICD-9 Diagnosis

ICD-9 Diagnosis

ICD-9 Diagnosis

ICD-9 Diagnosis

ICD-9 Diagnosis

ICD-9 Diagnosis

ICD-9 Diagnosis

ICD-9 Diagnosis

ICD-9 Diagnosis

ICD-9 Diagnosis

ICD-9 Diagnosis

continued on next page 
APPENDIX B Code Lists (continued)

\section{Category}

Serious infection

Serious infection

Serious infection

Serious infection

Serious infection

Serious infection

Serious infection

Serious infection

Serious infection

Serious infection

Serious infection

Serious infection

Serious infection

Serious infection

Serious infection

Serious infection

Serious infection

Serious infection

Serious infection

Serious infection

Serious infection

Serious infection

Serious infection

Serious infection

Serious infection

Serious infection

Serious infection

Serious infection

Serious infection

Serious infection

Serious infection

Serious infection

Serious infection

Serious infection

Serious infection

Serious infection

Serious infection

Serious infection

Serious infection

Serious infection

Serious infection

Serious infection

\section{Description}

Other

Other

Other

Other

Other

Other

Other

Other

Other

Other

Other

Other

Other

Other

Other

Other

Other

Other

Other

Other

Other

Other

Other

Other

Other

Other

Other

Other

Other

Other

Other

Other

Other

Other

Other

Other

Other

Other

Other

Other

Other

Other

\section{Code}

082.41

082.49

082.8

082.9

083.1

083.2

083.8

083.9

084.0

084.1

084.2

084.3

084.4

084.5

084.6

084.7

084.8

084.9

085.0

085.1

085.2

085.3

085.4

085.5

085.9

086.0

086.1

086.2

086.3

086.4

086.5

086.9

087.0

087.1

087.9

088.0

088.8

088.81

088.82

088.89

088.9

100.0
CodeType

ICD-9 Diagnosis

ICD-9 Diagnosis

ICD-9 Diagnosis

ICD-9 Diagnosis

ICD-9 Diagnosis

ICD-9 Diagnosis

ICD-9 Diagnosis

ICD-9 Diagnosis

ICD-9 Diagnosis

ICD-9 Diagnosis

ICD-9 Diagnosis

ICD-9 Diagnosis

ICD-9 Diagnosis

ICD-9 Diagnosis

ICD-9 Diagnosis

ICD-9 Diagnosis

ICD-9 Diagnosis

ICD-9 Diagnosis

ICD-9 Diagnosis

ICD-9 Diagnosis

ICD-9 Diagnosis

ICD-9 Diagnosis

ICD-9 Diagnosis

ICD-9 Diagnosis

ICD-9 Diagnosis

ICD-9 Diagnosis

ICD-9 Diagnosis

ICD-9 Diagnosis

ICD-9 Diagnosis

ICD-9 Diagnosis

ICD-9 Diagnosis

ICD-9 Diagnosis

ICD-9 Diagnosis

ICD-9 Diagnosis

ICD-9 Diagnosis

ICD-9 Diagnosis

ICD-9 Diagnosis

ICD-9 Diagnosis

ICD-9 Diagnosis

ICD-9 Diagnosis

ICD-9 Diagnosis

ICD-9 Diagnosis

continued on next page 
APPENDIX B Code Lists (continued)

\section{Category}

Serious infection

Serious infection

Serious infection

Serious infection

Serious infection

Serious infection

Serious infection

Serious infection

Serious infection

Serious infection

Serious infection

Serious infection

Serious infection

Serious infection

Serious infection

Serious infection

Serious infection

Serious infection

Serious infection

Serious infection

Serious infection

Serious infection

Serious infection

Serious infection

Serious infection

Serious infection

Serious infection

Serious infection

Serious infection

Serious infection

Serious infection

Serious infection

Serious infection

Serious infection

Serious infection

Serious infection

Serious infection

Serious infection

Serious infection

Serious infection

Serious infection

Serious infection

\section{Description}

Other

Other

Other

Other

Other

Other

Other

Other

Other

Other

Other

Other

Other

Other

Other

Other

Other

Other

Other

Other

Other

Other

Other

Other

Other

Other

Other

Other

Other

Other

Other

Other

Other

Other

Other

Other

Other

Other

Other

Other

Other

Other

\section{Code}

100.9

101

102.0

102.1

102.2

102.3

102.4

102.5

102.6

102.7

102.8

102.9

103.0

103.1

103.2

103.3

103.9

104.0

104.8

104.9

120.0

120.1

120.2

120.3

120.8

120.9

121.0

121.1

121.2

121.3

121.4

121.5

121.6

121.8

121.9

122.0

122.1

122.2

122.3

122.4

122.5

122.6
CodeType

ICD-9 Diagnosis

ICD-9 Diagnosis

ICD-9 Diagnosis

ICD-9 Diagnosis

ICD-9 Diagnosis

ICD-9 Diagnosis

ICD-9 Diagnosis

ICD-9 Diagnosis

ICD-9 Diagnosis

ICD-9 Diagnosis

ICD-9 Diagnosis

ICD-9 Diagnosis

ICD-9 Diagnosis

ICD-9 Diagnosis

ICD-9 Diagnosis

ICD-9 Diagnosis

ICD-9 Diagnosis

ICD-9 Diagnosis

ICD-9 Diagnosis

ICD-9 Diagnosis

ICD-9 Diagnosis

ICD-9 Diagnosis

ICD-9 Diagnosis

ICD-9 Diagnosis

ICD-9 Diagnosis

ICD-9 Diagnosis

ICD-9 Diagnosis

ICD-9 Diagnosis

ICD-9 Diagnosis

ICD-9 Diagnosis

ICD-9 Diagnosis

ICD-9 Diagnosis

ICD-9 Diagnosis

ICD-9 Diagnosis

ICD-9 Diagnosis

ICD-9 Diagnosis

ICD-9 Diagnosis

ICD-9 Diagnosis

ICD-9 Diagnosis

ICD-9 Diagnosis

ICD-9 Diagnosis

ICD-9 Diagnosis

continued on next page 
APPENDIX B Code Lists (continued)

\section{Category}

Serious infection

Serious infection

Serious infection

Serious infection

Serious infection

Serious infection

Serious infection

Serious infection

Serious infection

Serious infection

Serious infection

Serious infection

Serious infection

Serious infection

Serious infection

Serious infection

Serious infection

Serious infection

Serious infection

Serious infection

Serious infection

Serious infection

Serious infection

Serious infection

Serious infection

Serious infection

Serious infection

Serious infection

Serious infection

Serious infection

Serious infection

Serious infection

Serious infection

Serious infection

Serious infection

Serious infection

Serious infection

Serious infection

Serious infection

Serious infection

Serious infection

Serious infection

\section{Description}

Other

Other

Other

Other

Other

Other

Other

Other

Other

Other

Other

Other

Other

Other

Other

Other

Other

Other

Other

Other

Other

Other

Other

Other

Other

Other

Other

Other

Other

Other

Other

Other

Other

Other

Other

Other

Other

Other

Other

Other

Other

Other

\section{Code}

122.7

122.8

122.9

123.0

123.1

123.2

123.3

123.4

123.5

123.6

123.8

123.9

124

125.0

125.1

125.2

125.3

125.4

125.5

125.6

125.7

125.9

126.0

126.1

126.2

126.3

126.8

126.9

127.0

127.1

127.2

127.3

127.4

127.5

127.6

127.7

127.8

127.9

128.0

128.1

128.8

128.9
CodeType

ICD-9 Diagnosis

ICD-9 Diagnosis

ICD-9 Diagnosis

ICD-9 Diagnosis

ICD-9 Diagnosis

ICD-9 Diagnosis

ICD-9 Diagnosis

ICD-9 Diagnosis

ICD-9 Diagnosis

ICD-9 Diagnosis

ICD-9 Diagnosis

ICD-9 Diagnosis

ICD-9 Diagnosis

ICD-9 Diagnosis

ICD-9 Diagnosis

ICD-9 Diagnosis

ICD-9 Diagnosis

ICD-9 Diagnosis

ICD-9 Diagnosis

ICD-9 Diagnosis

ICD-9 Diagnosis

ICD-9 Diagnosis

ICD-9 Diagnosis

ICD-9 Diagnosis

ICD-9 Diagnosis

ICD-9 Diagnosis

ICD-9 Diagnosis

ICD-9 Diagnosis

ICD-9 Diagnosis

ICD-9 Diagnosis

ICD-9 Diagnosis

ICD-9 Diagnosis

ICD-9 Diagnosis

ICD-9 Diagnosis

ICD-9 Diagnosis

ICD-9 Diagnosis

ICD-9 Diagnosis

ICD-9 Diagnosis

ICD-9 Diagnosis

ICD-9 Diagnosis

ICD-9 Diagnosis

ICD-9 Diagnosis

continued on next page 
APPENDIX B Code Lists (continued)

\section{Category}

Serious infection

Serious infection

Serious infection

Serious infection

Serious infection

Serious infection

Serious infection

Serious infection

Serious infection

Serious infection

Serious infection

Serious infection

Serious infection

Serious infection

Serious infection

Serious infection

Serious infection

Serious infection

Serious infection

Serious infection

Serious infection

Serious infection

Serious infection

Serious infection

Serious infection

Serious infection

Serious infection

Serious infection

Serious infection

Serious infection

Serious infection

Serious infection

Serious infection

Serious infection

Serious infection

Serious infection

Serious infection

Serious infection

Serious infection

Serious infection

Serious infection

Serious infection

\section{Description}

Other

Other

Other

Other

Other

Other

Other

Other

Other

Other

Other

Other

Other

Other

Other

Other

Other

Other

Other

Other

Other

Other

Other

Other

Other

Other

Other

Other

Other

Other

Other

Other

Other

Other

Other

Other

Other

Other

Other

Other

Other

Peritonitis

\section{Code}

129

130.5

130.7

130.8

130.9

131.00

131.01

131.02

131.03

131.09

131.8

131.9

132.0

132.1

132.2

132.3

132.9

133.0

133.8

133.9

134.0

134.1

134.2

134.8

134.9

135

136.0

136.1

136.2

136.21

136.29

136.4

136.5

136.8

136.9

139.8

1008.9

V12.0

V12.00

V12.03

V12.09

032.83

\section{CodeType}

ICD-9 Diagnosis

ICD-9 Diagnosis

ICD-9 Diagnosis

ICD-9 Diagnosis

ICD-9 Diagnosis

ICD-9 Diagnosis

ICD-9 Diagnosis

ICD-9 Diagnosis

ICD-9 Diagnosis

ICD-9 Diagnosis

ICD-9 Diagnosis

ICD-9 Diagnosis

ICD-9 Diagnosis

ICD-9 Diagnosis

ICD-9 Diagnosis

ICD-9 Diagnosis

ICD-9 Diagnosis

ICD-9 Diagnosis

ICD-9 Diagnosis

ICD-9 Diagnosis

ICD-9 Diagnosis

ICD-9 Diagnosis

ICD-9 Diagnosis

ICD-9 Diagnosis

ICD-9 Diagnosis

ICD-9 Diagnosis

ICD-9 Diagnosis

ICD-9 Diagnosis

ICD-9 Diagnosis

ICD-9 Diagnosis

ICD-9 Diagnosis

ICD-9 Diagnosis

ICD-9 Diagnosis

ICD-9 Diagnosis

ICD-9 Diagnosis

ICD-9 Diagnosis

ICD-9 Diagnosis

ICD-9 Diagnosis

ICD-9 Diagnosis

ICD-9 Diagnosis

ICD-9 Diagnosis

ICD-9 Diagnosis

continued on next page 
APPENDIX B Code Lists (continued)

\section{Category}

Serious infection

Serious infection

Serious infection

Serious infection

Serious infection

Serious infection

Serious infection

Serious infection

Serious infection

Serious infection

Serious infection

Serious infection

Serious infection

Serious infection

Serious infection

Serious infection

Serious infection

Serious infection

Serious infection

Serious infection

Serious infection

Serious infection

Serious infection

Serious infection

Serious infection

Serious infection

Serious infection

Serious infection

Serious infection

Serious infection

Serious infection

Serious infection

Serious infection

Serious infection

Serious infection

Serious infection

Serious infection

Serious infection

Serious infection

Serious infection

Serious infection

Serious infection

\section{Description}

Peritonitis

Peritonitis

Peritonitis

Peritonitis

Peritonitis

Peritonitis

Peritonitis

Peritonitis

Peritonitis

Peritonitis

Peritonitis

Peritonitis

Peritonitis

Peritonitis

Peritonitis

Pneumonia

Pneumonia

Pneumonia

Pneumonia

Pneumonia

Pneumonia

Pneumonia

Pneumonia

Pneumonia

Pneumonia

Pneumonia

Pneumonia

Pneumonia

Pneumonia

Pneumonia

Pneumonia

Pneumonia

Pneumonia

Pneumonia

Pneumonia

Pneumonia

Pneumonia

Pneumonia

Pneumonia

Pneumonia

Pneumonia

Pneumonia

\section{Code}

567.0

567.1

567.2

567.21

567.22

567.23

567.29

567.38

567.39

567.8

567.81

567.82

567.89

567.9

569.5

003.22

020.3

020.4

020.5

021.2

022.1

031.0

039.1

052.1

055.1

073.0

083.0

112.4

114.0

114.4

114.5

115.05

115.15

115.95

130.4

136.3

480.0

480.1

480.2

480.3

480.8

480.9

\section{CodeType}

ICD-9 Diagnosis

ICD-9 Diagnosis

ICD-9 Diagnosis

ICD-9 Diagnosis

ICD-9 Diagnosis

ICD-9 Diagnosis

ICD-9 Diagnosis

ICD-9 Diagnosis

ICD-9 Diagnosis

ICD-9 Diagnosis

ICD-9 Diagnosis

ICD-9 Diagnosis

ICD-9 Diagnosis

ICD-9 Diagnosis

ICD-9 Diagnosis

ICD-9 Diagnosis

ICD-9 Diagnosis

ICD-9 Diagnosis

ICD-9 Diagnosis

ICD-9 Diagnosis

ICD-9 Diagnosis

ICD-9 Diagnosis

ICD-9 Diagnosis

ICD-9 Diagnosis

ICD-9 Diagnosis

ICD-9 Diagnosis

ICD-9 Diagnosis

ICD-9 Diagnosis

ICD-9 Diagnosis

ICD-9 Diagnosis

ICD-9 Diagnosis

ICD-9 Diagnosis

ICD-9 Diagnosis

ICD-9 Diagnosis

ICD-9 Diagnosis

ICD-9 Diagnosis

ICD-9 Diagnosis

ICD-9 Diagnosis

ICD-9 Diagnosis

ICD-9 Diagnosis

ICD-9 Diagnosis

ICD-9 Diagnosis

continued on next page 
APPENDIX B Code Lists (continued)

\section{Category}

Serious infection

Serious infection

Serious infection

Serious infection

Serious infection

Serious infection

Serious infection

Serious infection

Serious infection

Serious infection

Serious infection

Serious infection

Serious infection

Serious infection

Serious infection

Serious infection

Serious infection

Serious infection

Serious infection

Serious infection

Serious infection

Serious infection

Serious infection

Serious infection

Serious infection

Serious infection

Serious infection

Serious infection

Serious infection

Serious infection

Serious infection

Serious infection

Serious infection

Serious infection

Serious infection

Serious infection

Serious infection

Serious infection

Serious infection

Serious infection

Serious infection

Serious infection

\section{Description}

Pneumonia

Pneumonia

Pneumonia

Pneumonia

Pneumonia

Pneumonia

Pneumonia

Pneumonia

Pneumonia

Pneumonia

Pneumonia

Pneumonia

Pneumonia

Pneumonia

Pneumonia

Pneumonia

Pneumonia

Pneumonia

Pneumonia

Pneumonia

Pneumonia

Pneumonia

Pneumonia

Pneumonia

Pneumonia

Pneumonia

Pneumonia

Pneumonia

Pneumonia

Pneumonia

Pneumonia

Pneumonia

Pneumonia

Pneumonia

Pneumonia

Complication from procedures

Complication from procedures

Complication from procedures

Complication from procedures

Complication from procedures

Complication from procedures

Complication from procedures

\section{Code}

481

482.0

482.1

482.2

482.3

482.30

482.31

482.32

482.39

482.4

482.40

482.41

482.42

482.49

482.8

482.81

482.82

482.83

482.84

482.89

482.9

483

483.0

483.1

483.8

484.1

484.3

484.5

484.6

484.7

484.8

485

486

513.0

517.1

276.61

277.83

277.88

285.3

287.41

349.0

349.1
CodeType

ICD-9 Diagnosis

ICD-9 Diagnosis

ICD-9 Diagnosis

ICD-9 Diagnosis

ICD-9 Diagnosis

ICD-9 Diagnosis

ICD-9 Diagnosis

ICD-9 Diagnosis

ICD-9 Diagnosis

ICD-9 Diagnosis

ICD-9 Diagnosis

ICD-9 Diagnosis

ICD-9 Diagnosis

ICD-9 Diagnosis

ICD-9 Diagnosis

ICD-9 Diagnosis

ICD-9 Diagnosis

ICD-9 Diagnosis

ICD-9 Diagnosis

ICD-9 Diagnosis

ICD-9 Diagnosis

ICD-9 Diagnosis

ICD-9 Diagnosis

ICD-9 Diagnosis

ICD-9 Diagnosis

ICD-9 Diagnosis

ICD-9 Diagnosis

ICD-9 Diagnosis

ICD-9 Diagnosis

ICD-9 Diagnosis

ICD-9 Diagnosis

ICD-9 Diagnosis

ICD-9 Diagnosis

ICD-9 Diagnosis

ICD-9 Diagnosis

ICD-9 Diagnosis

ICD-9 Diagnosis

ICD-9 Diagnosis

ICD-9 Diagnosis

ICD-9 Diagnosis

ICD-9 Diagnosis

ICD-9 Diagnosis

continued on next page 
APPENDIX B Code Lists (continued)

\section{Category}

Serious infection

Serious infection

Serious infection

Serious infection

Serious infection

Serious infection

Serious infection

Serious infection

Serious infection

Serious infection

Serious infection

Serious infection

Serious infection

Serious infection

Serious infection

Serious infection

Serious infection

Serious infection

Serious infection

Serious infection

Serious infection

Serious infection

Serious infection

Serious infection

Serious infection

Serious infection

Serious infection

Serious infection

Serious infection

Serious infection

Serious infection

Serious infection

Serious infection

Serious infection

Serious infection

Serious infection

Serious infection

Serious infection

Serious infection

Serious infection

Serious infection

Serious infection

\section{Description}

Complication from procedures

Complication from procedures

Complication from procedures

Complication from procedures

Complication from procedures

Complication from procedures

Complication from procedures

Complication from procedures

Complication from procedures

Complication from procedures

Complication from procedures

Complication from procedures

Complication from procedures

Complication from procedures

Complication from procedures

Complication from procedures

Complication from procedures

Complication from procedures

Complication from procedures

Complication from procedures

Complication from procedures

Complication from procedures

Complication from procedures

Complication from procedures

Complication from procedures

Complication from procedures

Complication from procedures

Complication from procedures

Complication from procedures

Complication from procedures

Complication from procedures

Complication from procedures

Complication from procedures

Complication from procedures

Complication from procedures

Complication from procedures

Complication from procedures

Complication from procedures

Complication from procedures

Complication from procedures

Complication from procedures

Complication from procedures

\section{Code}

349.31

415.11

429.4

458.2

458.21

458.29

512.1

512.2

518.7

519.0

519.00

519.01

519.02

519.09

530.86

530.87

536.40

536.41

536.42

536.49

539.01

539.09

539.81

539.89

564.2

564.3

564.4

569.6

569.62

569.71

569.79

579.3

596.81

780.62

780.63

780.66

909.3

995.24

995.4

995.86

997.0

997.00
CodeType

ICD-9 Diagnosis

ICD-9 Diagnosis

ICD-9 Diagnosis

ICD-9 Diagnosis

ICD-9 Diagnosis

ICD-9 Diagnosis

ICD-9 Diagnosis

ICD-9 Diagnosis

ICD-9 Diagnosis

ICD-9 Diagnosis

ICD-9 Diagnosis

ICD-9 Diagnosis

ICD-9 Diagnosis

ICD-9 Diagnosis

ICD-9 Diagnosis

ICD-9 Diagnosis

ICD-9 Diagnosis

ICD-9 Diagnosis

ICD-9 Diagnosis

ICD-9 Diagnosis

ICD-9 Diagnosis

ICD-9 Diagnosis

ICD-9 Diagnosis

ICD-9 Diagnosis

ICD-9 Diagnosis

ICD-9 Diagnosis

ICD-9 Diagnosis

ICD-9 Diagnosis

ICD-9 Diagnosis

ICD-9 Diagnosis

ICD-9 Diagnosis

ICD-9 Diagnosis

ICD-9 Diagnosis

ICD-9 Diagnosis

ICD-9 Diagnosis

ICD-9 Diagnosis

ICD-9 Diagnosis

ICD-9 Diagnosis

ICD-9 Diagnosis

ICD-9 Diagnosis

ICD-9 Diagnosis

ICD-9 Diagnosis

continued on next page 
APPENDIX B Code Lists (continued)

\section{Category}

Serious infection

Serious infection

Serious infection

Serious infection

Serious infection

Serious infection

Serious infection

Serious infection

Serious infection

Serious infection

Serious infection

Serious infection

Serious infection

Serious infection

Serious infection

Serious infection

Serious infection

Serious infection

Serious infection

Serious infection

Serious infection

Serious infection

Serious infection

Serious infection

Serious infection

Serious infection

Serious infection

Serious infection

Serious infection

Serious infection

Serious infection

Serious infection

Serious infection

Serious infection

Serious infection

Serious infection

Serious infection

Serious infection

Serious infection

Serious infection

Serious infection

Serious infection

\section{Description}

Complication from procedures

Complication from procedures

Complication from procedures

Complication from procedures

Complication from procedures

Complication from procedures

Complication from procedures

Complication from procedures

Complication from procedures

Complication from procedures

Complication from procedures

Complication from procedures

Complication from procedures

Complication from procedures

Complication from procedures

Complication from procedures

Complication from procedures

Complication from procedures

Complication from procedures

Complication from procedures

Complication from procedures

Complication from procedures

Complication from procedures

Complication from procedures

Complication from procedures

Complication from procedures

Complication from procedures

Complication from procedures

Complication from procedures

Complication from procedures

Complication from procedures

Complication from procedures

Complication from procedures

Complication from procedures

Complication from procedures

Complication from procedures

Complication from procedures

Complication from procedures

Complication from procedures

Complication from procedures

Complication from procedures

Complication from procedures

\section{Code}

997.01

997.02

997.09

997.1

997.2

997.3

997.31

997.32

997.39

997.4

997.41

997.49

997.5

997.60

997.61

997.62

997.69

997.71

997.72

997.79

997.9

997.91

997.99

998.0

998.00

998.01

998.02

998.09

998.1

998.11

998.12

998.13

998.2

998.3

998.30

998.31

998.32

998.33

998.4

998.5

998.51

998.59

\section{CodeType}

ICD-9 Diagnosis

ICD-9 Diagnosis

ICD-9 Diagnosis

ICD-9 Diagnosis

ICD-9 Diagnosis

ICD-9 Diagnosis

ICD-9 Diagnosis

ICD-9 Diagnosis

ICD-9 Diagnosis

ICD-9 Diagnosis

ICD-9 Diagnosis

ICD-9 Diagnosis

ICD-9 Diagnosis

ICD-9 Diagnosis

ICD-9 Diagnosis

ICD-9 Diagnosis

ICD-9 Diagnosis

ICD-9 Diagnosis

ICD-9 Diagnosis

ICD-9 Diagnosis

ICD-9 Diagnosis

ICD-9 Diagnosis

ICD-9 Diagnosis

ICD-9 Diagnosis

ICD-9 Diagnosis

ICD-9 Diagnosis

ICD-9 Diagnosis

ICD-9 Diagnosis

ICD-9 Diagnosis

ICD-9 Diagnosis

ICD-9 Diagnosis

ICD-9 Diagnosis

ICD-9 Diagnosis

ICD-9 Diagnosis

ICD-9 Diagnosis

ICD-9 Diagnosis

ICD-9 Diagnosis

ICD-9 Diagnosis

ICD-9 Diagnosis

ICD-9 Diagnosis

ICD-9 Diagnosis

ICD-9 Diagnosis

continued on next page 
APPENDIX B Code Lists (continued)

\section{Category}

Serious infection

Serious infection

Serious infection

Serious infection

Serious infection

Serious infection

Serious infection

Serious infection

Serious infection

Serious infection

Serious infection

Serious infection

Serious infection

Serious infection

Serious infection

Serious infection

Serious infection

Serious infection

Serious infection

Serious infection

Serious infection

Serious infection

Serious infection

Serious infection

Serious infection

Serious infection

Serious infection

Serious infection

Serious infection

Serious infection

Serious infection

Serious infection

Serious infection

Serious infection

Serious infection

Serious infection

Serious infection

Serious infection

Serious infection

Serious infection

Serious infection

Serious infection

\section{Description}

Complication from procedures

Complication from procedures

Complication from procedures

Complication from procedures

Complication from procedures

Complication from procedures

Complication from procedures

Complication from procedures

Complication from procedures

Complication from procedures

Complication from procedures

Complication from procedures

Complication from procedures

Complication from procedures

Complication from procedures

Complication from procedures

Complication from procedures

Complication from procedures

Complication from procedures

Complication from procedures

Complication from procedures

Complication from procedures

Complication from procedures

Complication from procedures

Complication from procedures

Complication from procedures

Complication from procedures

Complication from procedures

Complication from procedures

Complication from procedures

Complication from procedures

Complication from procedures

Complication from procedures

Complication from procedures

Complication from procedures

Complication from procedures

Complication from procedures

Complication from procedures

Complication from procedures

Complication from procedures

Complication from procedures

Complication from procedures

\section{Code}

998.6

998.7

998.8

998.81

998.82

998.83

998.89

998.9

999.0

999.1

999.2

999.3

999.34

999.39

999.4

999.41

999.42

999.49

999.5

999.51

999.52

999.59

999.6

999.60

999.61

999.62

999.63

999.69

999.7

999.70

999.71

999.72

999.73

999.74

999.75

999.76

999.77

999.78

999.79

999.8

999.80

999.81

\section{CodeType}

ICD-9 Diagnosis

ICD-9 Diagnosis

ICD-9 Diagnosis

ICD-9 Diagnosis

ICD-9 Diagnosis

ICD-9 Diagnosis

ICD-9 Diagnosis

ICD-9 Diagnosis

ICD-9 Diagnosis

ICD-9 Diagnosis

ICD-9 Diagnosis

ICD-9 Diagnosis

ICD-9 Diagnosis

ICD-9 Diagnosis

ICD-9 Diagnosis

ICD-9 Diagnosis

ICD-9 Diagnosis

ICD-9 Diagnosis

ICD-9 Diagnosis

ICD-9 Diagnosis

ICD-9 Diagnosis

ICD-9 Diagnosis

ICD-9 Diagnosis

ICD-9 Diagnosis

ICD-9 Diagnosis

ICD-9 Diagnosis

ICD-9 Diagnosis

ICD-9 Diagnosis

ICD-9 Diagnosis

ICD-9 Diagnosis

ICD-9 Diagnosis

ICD-9 Diagnosis

ICD-9 Diagnosis

ICD-9 Diagnosis

ICD-9 Diagnosis

ICD-9 Diagnosis

ICD-9 Diagnosis

ICD-9 Diagnosis

ICD-9 Diagnosis

ICD-9 Diagnosis

ICD-9 Diagnosis

ICD-9 Diagnosis

continued on next page 
APPENDIX B Code Lists (continued)

\section{Category}

Serious infection

Serious infection

Serious infection

Serious infection

Serious infection

Serious infection

Serious infection

Serious infection

Serious infection

Serious infection

Serious infection

Serious infection

Serious infection

Serious infection

Serious infection

Serious infection

Serious infection

Serious infection

Serious infection

Serious infection

Serious infection

Serious infection

Serious infection

Serious infection

Serious infection

Serious infection

Serious infection

Serious infection

Serious infection

Serious infection

Serious infection

Serious infection

Serious infection

Serious infection

Serious infection

Serious infection

Serious infection

Serious infection

Serious infection

Serious infection

Serious infection

Serious infection

\section{Description}

Complication from procedures

Complication from procedures

Complication from procedures

Complication from procedures

Complication from procedures

Complication from procedures

Complication from procedures

Complication from procedures

Complication from procedures

Complication from procedures

Complication from procedures

Complication from procedures

Septicemia

Septicemia

Septicemia

Septicemia

Septicemia

Septicemia

Septicemia

Septicemia

Septicemia

Septicemia

Septicemia

Septicemia

Septicemia

Septicemia

Septicemia

Septicemia

Septicemia

Septicemia

Septicemia

Septicemia

Septicemia

Septicemia

Septicemia

Septicemia

Septicemia

Shock

Shock

Shock

Shock

SKIN

\section{Code}

999.82

999.83

999.84

999.85

999.88

999.89

999.9

V15.53

V15.80

V15.83

V90.01

V90.09

003.1

020.2

022.3

036.2

038.0

038.1

038.10

038.11

038.12

038.19

038.2

038.3

038.40

038.41

038.42

038.43

038.44

038.49

038.8

038.9

054.5

449

790.7

995.91

995.92

785.50

785.51

785.52

785.59

020.1

\section{CodeType}

ICD-9 Diagnosis

ICD-9 Diagnosis

ICD-9 Diagnosis

ICD-9 Diagnosis

ICD-9 Diagnosis

ICD-9 Diagnosis

ICD-9 Diagnosis

ICD-9 Diagnosis

ICD-9 Diagnosis

ICD-9 Diagnosis

ICD-9 Diagnosis

ICD-9 Diagnosis

ICD-9 Diagnosis

ICD-9 Diagnosis

ICD-9 Diagnosis

ICD-9 Diagnosis

ICD-9 Diagnosis

ICD-9 Diagnosis

ICD-9 Diagnosis

ICD-9 Diagnosis

ICD-9 Diagnosis

ICD-9 Diagnosis

ICD-9 Diagnosis

ICD-9 Diagnosis

ICD-9 Diagnosis

ICD-9 Diagnosis

ICD-9 Diagnosis

ICD-9 Diagnosis

ICD-9 Diagnosis

ICD-9 Diagnosis

ICD-9 Diagnosis

ICD-9 Diagnosis

ICD-9 Diagnosis

ICD-9 Diagnosis

ICD-9 Diagnosis

ICD-9 Diagnosis

ICD-9 Diagnosis

ICD-9 Diagnosis

ICD-9 Diagnosis

ICD-9 Diagnosis

ICD-9 Diagnosis

ICD-9 Diagnosis

continued on next page 
APPENDIX B Code Lists (continued)

\section{Category}

Serious infection

Serious infection

Serious infection

Serious infection

Serious infection

Serious infection

Serious infection

Serious infection

Serious infection

Serious infection

Serious infection

Serious infection

Serious infection

Serious infection

Serious infection

Serious infection

Serious infection

Serious infection

Serious infection

Serious infection

Serious infection

Serious infection

Serious infection

Serious infection

Serious infection

Serious infection

Serious infection

Serious infection

Serious infection

Serious infection

Serious infection

Serious infection

Serious infection

Serious infection

Serious infection

Serious infection

Serious infection

Serious infection

Serious infection

Serious infection

Serious infection

Serious infection

\section{Description}

SKIN

SKIN

SKIN

SKIN

SKIN

SKIN

SKIN

SKIN

SKIN

SKIN

SKIN

SKIN

SKIN

SKIN

SKIN

SKIN

SKIN

SKIN

SKIN

SKIN

SKIN

SKIN

SKIN

SKIN

SKIN

SKIN

SKIN

SKIN

SKIN

SKIN

SKIN

SKIN

SKIN

SKIN

SKIN

SKIN

SKIN

SKIN

SKIN

SKIN

SKIN

SKIN
Code

021.0

022.0

031.1

032.85

035

039.0

680.0

680.1

680.2

680.3

680.4

680.5

680.6

680.7

680.8

680.9

681.00

681.01

681.02

681.10

681.11

681.9

682.0

682.1

682.2

682.3

682.4

682.5

682.6

682.7

682.8

682.9

684

685.0

685.1

686.0

686.00

686.01

686.09

686.1

686.8

686.9
CodeType

ICD-9 Diagnosis

ICD-9 Diagnosis

ICD-9 Diagnosis

ICD-9 Diagnosis

ICD-9 Diagnosis

ICD-9 Diagnosis

ICD-9 Diagnosis

ICD-9 Diagnosis

ICD-9 Diagnosis

ICD-9 Diagnosis

ICD-9 Diagnosis

ICD-9 Diagnosis

ICD-9 Diagnosis

ICD-9 Diagnosis

ICD-9 Diagnosis

ICD-9 Diagnosis

ICD-9 Diagnosis

ICD-9 Diagnosis

ICD-9 Diagnosis

ICD-9 Diagnosis

ICD-9 Diagnosis

ICD-9 Diagnosis

ICD-9 Diagnosis

ICD-9 Diagnosis

ICD-9 Diagnosis

ICD-9 Diagnosis

ICD-9 Diagnosis

ICD-9 Diagnosis

ICD-9 Diagnosis

ICD-9 Diagnosis

ICD-9 Diagnosis

ICD-9 Diagnosis

ICD-9 Diagnosis

ICD-9 Diagnosis

ICD-9 Diagnosis

ICD-9 Diagnosis

ICD-9 Diagnosis

ICD-9 Diagnosis

ICD-9 Diagnosis

ICD-9 Diagnosis

ICD-9 Diagnosis

ICD-9 Diagnosis

continued on next page 
APPENDIX B Code Lists (continued)

\section{Category}

Serious infection

Serious infection

Serious infection

Serious infection

Serious infection

Serious infection

Serious infection

Serious infection

Serious infection

Serious infection

Serious infection

Serious infection

Serious infection

Serious infection

Serious infection

Serious infection

Serious infection

Serious infection

Serious infection

Serious infection

Serious infection

Serious infection

Serious infection

Serious infection

Serious infection

Serious infection

Serious infection

Serious infection

Serious infection

Serious infection

Serious infection

Serious infection

Serious infection

Serious infection

Serious infection

Serious infection

Serious infection

Serious infection

Serious infection

Serious infection

Serious infection

Serious infection

\section{Description}

STD

STD

STD

STD

STD

STD

STD

STD

STD

STD

STD

STD

STD

STD

STD

STD

STD

STD

STD

STD

STD

STD

STD

STD

STD

STD

STD

STD

STD

STD

STD

STD

STD

STD

STD

STD

STD

STD

STD

STD

STD

STD

\section{Code}

090.0

090.1

090.2

090.3

090.40

090.41

090.42

090.49

090.5

090.6

090.7

090.9

091.0

091.1

091.2

091.3

091.4

091.50

091.51

091.52

091.61

091.62

091.69

091.7

091.81

091.82

091.89

091.9

092.0

092.9

093.0

093.1

093.20

093.21

093.22

093.23

093.24

093.81

093.82

093.89

093.9

094.0
CodeType

ICD-9 Diagnosis

ICD-9 Diagnosis

ICD-9 Diagnosis

ICD-9 Diagnosis

ICD-9 Diagnosis

ICD-9 Diagnosis

ICD-9 Diagnosis

ICD-9 Diagnosis

ICD-9 Diagnosis

ICD-9 Diagnosis

ICD-9 Diagnosis

ICD-9 Diagnosis

ICD-9 Diagnosis

ICD-9 Diagnosis

ICD-9 Diagnosis

ICD-9 Diagnosis

ICD-9 Diagnosis

ICD-9 Diagnosis

ICD-9 Diagnosis

ICD-9 Diagnosis

ICD-9 Diagnosis

ICD-9 Diagnosis

ICD-9 Diagnosis

ICD-9 Diagnosis

ICD-9 Diagnosis

ICD-9 Diagnosis

ICD-9 Diagnosis

ICD-9 Diagnosis

ICD-9 Diagnosis

ICD-9 Diagnosis

ICD-9 Diagnosis

ICD-9 Diagnosis

ICD-9 Diagnosis

ICD-9 Diagnosis

ICD-9 Diagnosis

ICD-9 Diagnosis

ICD-9 Diagnosis

ICD-9 Diagnosis

ICD-9 Diagnosis

ICD-9 Diagnosis

ICD-9 Diagnosis

ICD-9 Diagnosis

continued on next page 
APPENDIX B Code Lists (continued)

\section{Category}

Serious infection

Serious infection

Serious infection

Serious infection

Serious infection

Serious infection

Serious infection

Serious infection

Serious infection

Serious infection

Serious infection

Serious infection

Serious infection

Serious infection

Serious infection

Serious infection

Serious infection

Serious infection

Serious infection

Serious infection

Serious infection

Serious infection

Serious infection

Serious infection

Serious infection

Serious infection

Serious infection

Serious infection

Serious infection

Serious infection

Serious infection

Serious infection

Serious infection

Serious infection

Serious infection

Serious infection

Serious infection

Serious infection

Serious infection

Serious infection

Serious infection

Serious infection

\section{Description}

STD

STD

STD

STD

STD

STD

STD

STD

STD

STD

STD

STD

STD

STD

STD

STD

STD

STD

STD

STD

STD

STD

STD

STD

STD

STD

STD

STD

STD

STD

STD

STD

STD

STD

STD

STD

STD

STD

STD

STD

STD

STD

\section{Code}

094.1

094.2

094.3

094.81

094.82

094.83

094.84

094.85

094.86

094.87

094.89

094.9

095.0

095.1

095.2

095.3

095.4

095.5

095.6

095.7

095.8

095.9

096

097.0

097.1

097.9

098.0

098.10

098.11

098.12

098.13

098.14

098.15

098.16

098.17

098.19

098.2

098.30

098.31

098.32

098.33

098.34
CodeType

ICD-9 Diagnosis

ICD-9 Diagnosis

ICD-9 Diagnosis

ICD-9 Diagnosis

ICD-9 Diagnosis

ICD-9 Diagnosis

ICD-9 Diagnosis

ICD-9 Diagnosis

ICD-9 Diagnosis

ICD-9 Diagnosis

ICD-9 Diagnosis

ICD-9 Diagnosis

ICD-9 Diagnosis

ICD-9 Diagnosis

ICD-9 Diagnosis

ICD-9 Diagnosis

ICD-9 Diagnosis

ICD-9 Diagnosis

ICD-9 Diagnosis

ICD-9 Diagnosis

ICD-9 Diagnosis

ICD-9 Diagnosis

ICD-9 Diagnosis

ICD-9 Diagnosis

ICD-9 Diagnosis

ICD-9 Diagnosis

ICD-9 Diagnosis

ICD-9 Diagnosis

ICD-9 Diagnosis

ICD-9 Diagnosis

ICD-9 Diagnosis

ICD-9 Diagnosis

ICD-9 Diagnosis

ICD-9 Diagnosis

ICD-9 Diagnosis

ICD-9 Diagnosis

ICD-9 Diagnosis

ICD-9 Diagnosis

ICD-9 Diagnosis

ICD-9 Diagnosis

ICD-9 Diagnosis

ICD-9 Diagnosis

continued on next page 
APPENDIX B Code Lists (continued)

\section{Category}

Serious infection

Serious infection

Serious infection

Serious infection

Serious infection

Serious infection

Serious infection

Serious infection

Serious infection

Serious infection

Serious infection

Serious infection

Serious infection

Serious infection

Serious infection

Serious infection

Serious infection

Serious infection

Serious infection

Serious infection

Serious infection

Serious infection

Serious infection

Serious infection

Serious infection

Serious infection

Serious infection

Serious infection

Serious infection

Serious infection

Serious infection

Serious infection

Serious infection

Serious infection

Serious infection

Serious infection

Serious infection

Serious infection

Serious infection

Serious infection

Serious infection

Serious infection

\section{Description}

STD

STD

STD

STD

STD

STD

STD

STD

STD

STD

STD

STD

STD

STD

STD

STD

STD

STD

STD

STD

STD

STD

STD

STD

STD

STD

STD

STD

STD

STD

STD

STD

STD

STD

STD

STD

STD

STD

STD

STD

STD

STD

\section{Code}

098.35

098.36

098.37

098.39

098.40

098.41

098.42

098.43

098.49

098.50

098.51

098.52

098.53

098.59

098.6

098.7

098.81

098.82

098.83

098.84

098.85

098.86

098.89

099.0

099.1

099.2

099.3

099.4

099.40

099.41

099.49

099.50

099.51

099.52

099.53

099.54

099.55

099.56

099.59

099.8

099.9

795.05
CodeType

ICD-9 Diagnosis

ICD-9 Diagnosis

ICD-9 Diagnosis

ICD-9 Diagnosis

ICD-9 Diagnosis

ICD-9 Diagnosis

ICD-9 Diagnosis

ICD-9 Diagnosis

ICD-9 Diagnosis

ICD-9 Diagnosis

ICD-9 Diagnosis

ICD-9 Diagnosis

ICD-9 Diagnosis

ICD-9 Diagnosis

ICD-9 Diagnosis

ICD-9 Diagnosis

ICD-9 Diagnosis

ICD-9 Diagnosis

ICD-9 Diagnosis

ICD-9 Diagnosis

ICD-9 Diagnosis

ICD-9 Diagnosis

ICD-9 Diagnosis

ICD-9 Diagnosis

ICD-9 Diagnosis

ICD-9 Diagnosis

ICD-9 Diagnosis

ICD-9 Diagnosis

ICD-9 Diagnosis

ICD-9 Diagnosis

ICD-9 Diagnosis

ICD-9 Diagnosis

ICD-9 Diagnosis

ICD-9 Diagnosis

ICD-9 Diagnosis

ICD-9 Diagnosis

ICD-9 Diagnosis

ICD-9 Diagnosis

ICD-9 Diagnosis

ICD-9 Diagnosis

ICD-9 Diagnosis

ICD-9 Diagnosis

continued on next page 
APPENDIX B Code Lists (continued)

\section{Category}

Serious infection

Serious infection

Serious infection

Serious infection

Serious infection

Serious infection

Serious infection

Serious infection

Serious infection

Serious infection

Serious infection

Serious infection

Serious infection

Serious infection

Serious infection

Serious infection

Serious infection

Serious infection

Serious infection

Serious infection

Serious infection

Serious infection

Serious infection

Serious infection

Serious infection

Serious infection

Serious infection

Serious infection

Serious infection

Serious infection

Serious infection

Serious infection

Serious infection

Serious infection

Serious infection

Serious infection

Serious infection

Serious infection

Serious infection

Serious infection

Serious infection

Serious infection

\section{Description}

STD

STD

STD

STD

TB

$\mathrm{TB}$

TB

TB

TB

TB

TB

TB

TB

TB

TB

TB

TB

TB

TB

TB

TB

TB

TB

TB

TB

TB

TB

TB

TB

TB

TB

TB

TB

TB

TB

TB

TB

TB

TB

TB

TB

TB
Code

795.15

795.19

796.75

796.79

010.00

010.01

010.02

010.03

010.04

010.05

010.06

010.10

010.11

010.12

010.13

010.14

010.15

010.16

010.80

010.81

010.82

010.83

010.84

010.85

010.86

010.90

010.91

010.92

010.93

010.94

010.95

010.96

011.00

011.01

011.02

011.03

011.04

011.05

011.06

011.10

011.11

011.12
CodeType

ICD-9 Diagnosis

ICD-9 Diagnosis

ICD-9 Diagnosis

ICD-9 Diagnosis

ICD-9 Diagnosis

ICD-9 Diagnosis

ICD-9 Diagnosis

ICD-9 Diagnosis

ICD-9 Diagnosis

ICD-9 Diagnosis

ICD-9 Diagnosis

ICD-9 Diagnosis

ICD-9 Diagnosis

ICD-9 Diagnosis

ICD-9 Diagnosis

ICD-9 Diagnosis

ICD-9 Diagnosis

ICD-9 Diagnosis

ICD-9 Diagnosis

ICD-9 Diagnosis

ICD-9 Diagnosis

ICD-9 Diagnosis

ICD-9 Diagnosis

ICD-9 Diagnosis

ICD-9 Diagnosis

ICD-9 Diagnosis

ICD-9 Diagnosis

ICD-9 Diagnosis

ICD-9 Diagnosis

ICD-9 Diagnosis

ICD-9 Diagnosis

ICD-9 Diagnosis

ICD-9 Diagnosis

ICD-9 Diagnosis

ICD-9 Diagnosis

ICD-9 Diagnosis

ICD-9 Diagnosis

ICD-9 Diagnosis

ICD-9 Diagnosis

ICD-9 Diagnosis

ICD-9 Diagnosis

ICD-9 Diagnosis

continued on next page 
APPENDIX B Code Lists (continued)

\section{Category}

Serious infection

Serious infection

Serious infection

Serious infection

Serious infection

Serious infection

Serious infection

Serious infection

Serious infection

Serious infection

Serious infection

Serious infection

Serious infection

Serious infection

Serious infection

Serious infection

Serious infection

Serious infection

Serious infection

Serious infection

Serious infection

Serious infection

Serious infection

Serious infection

Serious infection

Serious infection

Serious infection

Serious infection

Serious infection

Serious infection

Serious infection

Serious infection

Serious infection

Serious infection

Serious infection

Serious infection

Serious infection

Serious infection

Serious infection

Serious infection

Serious infection

Serious infection

\section{Description}

TB

TB

TB

TB

TB

TB

TB

TB

TB

TB

TB

TB

TB

TB

TB

TB

TB

TB

TB

TB

TB

TB

TB

TB

TB

TB

TB

TB

TB

TB

TB

TB

TB

TB

TB

TB

TB

TB

TB

TB

TB

TB

\section{Code}

011.13

011.14

011.15

011.16

011.20

011.21

011.22

011.23

011.24

011.25

011.26

011.30

011.31

011.32

011.33

011.34

011.35

011.36

011.40

011.41

011.42

011.43

011.44

011.45

011.46

011.50

011.51

011.52

011.53

011.54

011.55

011.56

011.60

011.61

011.62

011.63

011.64

011.65

011.66

011.70

011.71

011.72
CodeType

ICD-9 Diagnosis

ICD-9 Diagnosis

ICD-9 Diagnosis

ICD-9 Diagnosis

ICD-9 Diagnosis

ICD-9 Diagnosis

ICD-9 Diagnosis

ICD-9 Diagnosis

ICD-9 Diagnosis

ICD-9 Diagnosis

ICD-9 Diagnosis

ICD-9 Diagnosis

ICD-9 Diagnosis

ICD-9 Diagnosis

ICD-9 Diagnosis

ICD-9 Diagnosis

ICD-9 Diagnosis

ICD-9 Diagnosis

ICD-9 Diagnosis

ICD-9 Diagnosis

ICD-9 Diagnosis

ICD-9 Diagnosis

ICD-9 Diagnosis

ICD-9 Diagnosis

ICD-9 Diagnosis

ICD-9 Diagnosis

ICD-9 Diagnosis

ICD-9 Diagnosis

ICD-9 Diagnosis

ICD-9 Diagnosis

ICD-9 Diagnosis

ICD-9 Diagnosis

ICD-9 Diagnosis

ICD-9 Diagnosis

ICD-9 Diagnosis

ICD-9 Diagnosis

ICD-9 Diagnosis

ICD-9 Diagnosis

ICD-9 Diagnosis

ICD-9 Diagnosis

ICD-9 Diagnosis

ICD-9 Diagnosis

continued on next page 
APPENDIX B Code Lists (continued)

\section{Category}

Serious infection

Serious infection

Serious infection

Serious infection

Serious infection

Serious infection

Serious infection

Serious infection

Serious infection

Serious infection

Serious infection

Serious infection

Serious infection

Serious infection

Serious infection

Serious infection

Serious infection

Serious infection

Serious infection

Serious infection

Serious infection

Serious infection

Serious infection

Serious infection

Serious infection

Serious infection

Serious infection

Serious infection

Serious infection

Serious infection

Serious infection

Serious infection

Serious infection

Serious infection

Serious infection

Serious infection

Serious infection

Serious infection

Serious infection

Serious infection

Serious infection

Serious infection

\section{Description}

TB

TB

TB

TB

TB

TB

TB

TB

TB

TB

TB

TB

TB

TB

TB

TB

TB

TB

TB

TB

TB

TB

TB

TB

TB

TB

TB

TB

TB

TB

TB

TB

TB

TB

TB

TB

TB

TB

TB

TB

TB

TB

\section{Code}

011.73

011.74

011.75

011.76

011.80

011.81

011.82

011.83

011.84

011.85

011.86

011.90

011.91

011.92

011.93

011.94

011.95

011.96

012.00

012.01

012.02

012.03

012.04

012.05

012.06

012.10

012.11

012.12

012.13

012.14

012.15

012.16

012.20

012.21

012.22

012.23

012.24

012.25

012.26

012.30

012.31

012.32
CodeType

ICD-9 Diagnosis

ICD-9 Diagnosis

ICD-9 Diagnosis

ICD-9 Diagnosis

ICD-9 Diagnosis

ICD-9 Diagnosis

ICD-9 Diagnosis

ICD-9 Diagnosis

ICD-9 Diagnosis

ICD-9 Diagnosis

ICD-9 Diagnosis

ICD-9 Diagnosis

ICD-9 Diagnosis

ICD-9 Diagnosis

ICD-9 Diagnosis

ICD-9 Diagnosis

ICD-9 Diagnosis

ICD-9 Diagnosis

ICD-9 Diagnosis

ICD-9 Diagnosis

ICD-9 Diagnosis

ICD-9 Diagnosis

ICD-9 Diagnosis

ICD-9 Diagnosis

ICD-9 Diagnosis

ICD-9 Diagnosis

ICD-9 Diagnosis

ICD-9 Diagnosis

ICD-9 Diagnosis

ICD-9 Diagnosis

ICD-9 Diagnosis

ICD-9 Diagnosis

ICD-9 Diagnosis

ICD-9 Diagnosis

ICD-9 Diagnosis

ICD-9 Diagnosis

ICD-9 Diagnosis

ICD-9 Diagnosis

ICD-9 Diagnosis

ICD-9 Diagnosis

ICD-9 Diagnosis

ICD-9 Diagnosis

continued on next page 
APPENDIX B Code Lists (continued)

\section{Category}

Serious infection

Serious infection

Serious infection

Serious infection

Serious infection

Serious infection

Serious infection

Serious infection

Serious infection

Serious infection

Serious infection

Serious infection

Serious infection

Serious infection

Serious infection

Serious infection

Serious infection

Serious infection

Serious infection

Serious infection

Serious infection

Serious infection

Serious infection

Serious infection

Serious infection

Serious infection

Serious infection

Serious infection

Serious infection

Serious infection

Serious infection

Serious infection

Serious infection

Serious infection

Serious infection

Serious infection

Serious infection

Serious infection

Serious infection

Serious infection

Serious infection

Serious infection

\section{Description}

TB

TB

TB

TB

TB

TB

TB

TB

TB

TB

TB

TB

TB

TB

TB

TB

TB

TB

TB

TB

TB

TB

TB

TB

TB

TB

TB

TB

TB

TB

TB

TB

TB

TB

TB

TB

TB

TB

TB

TB

TB

TB

\section{Code}

012.33

012.34

012.35

012.36

012.80

012.81

012.82

012.83

012.84

012.85

012.86

013.00

013.01

013.02

013.03

013.04

013.05

013.06

013.10

013.11

013.12

013.13

013.14

013.15

013.16

013.20

013.21

013.22

013.23

013.24

013.25

013.26

013.30

013.31

013.32

013.33

013.34

013.35

013.36

013.40

013.41

013.42
CodeType

ICD-9 Diagnosis

ICD-9 Diagnosis

ICD-9 Diagnosis

ICD-9 Diagnosis

ICD-9 Diagnosis

ICD-9 Diagnosis

ICD-9 Diagnosis

ICD-9 Diagnosis

ICD-9 Diagnosis

ICD-9 Diagnosis

ICD-9 Diagnosis

ICD-9 Diagnosis

ICD-9 Diagnosis

ICD-9 Diagnosis

ICD-9 Diagnosis

ICD-9 Diagnosis

ICD-9 Diagnosis

ICD-9 Diagnosis

ICD-9 Diagnosis

ICD-9 Diagnosis

ICD-9 Diagnosis

ICD-9 Diagnosis

ICD-9 Diagnosis

ICD-9 Diagnosis

ICD-9 Diagnosis

ICD-9 Diagnosis

ICD-9 Diagnosis

ICD-9 Diagnosis

ICD-9 Diagnosis

ICD-9 Diagnosis

ICD-9 Diagnosis

ICD-9 Diagnosis

ICD-9 Diagnosis

ICD-9 Diagnosis

ICD-9 Diagnosis

ICD-9 Diagnosis

ICD-9 Diagnosis

ICD-9 Diagnosis

ICD-9 Diagnosis

ICD-9 Diagnosis

ICD-9 Diagnosis

ICD-9 Diagnosis

continued on next page 
APPENDIX B Code Lists (continued)

\section{Category}

Serious infection

Serious infection

Serious infection

Serious infection

Serious infection

Serious infection

Serious infection

Serious infection

Serious infection

Serious infection

Serious infection

Serious infection

Serious infection

Serious infection

Serious infection

Serious infection

Serious infection

Serious infection

Serious infection

Serious infection

Serious infection

Serious infection

Serious infection

Serious infection

Serious infection

Serious infection

Serious infection

Serious infection

Serious infection

Serious infection

Serious infection

Serious infection

Serious infection

Serious infection

Serious infection

Serious infection

Serious infection

Serious infection

Serious infection

Serious infection

Serious infection

Serious infection

\section{Description}

TB

TB

TB

TB

TB

TB

TB

TB

TB

TB

TB

TB

TB

TB

TB

TB

TB

TB

TB

TB

TB

TB

TB

TB

TB

TB

TB

TB

TB

TB

TB

TB

TB

TB

TB

TB

TB

TB

TB

TB

TB

TB

\section{Code}

013.43

013.44

013.45

013.46

013.50

013.51

013.52

013.53

013.54

013.55

013.56

013.60

013.61

013.62

013.63

013.64

013.65

013.66

013.80

013.81

013.82

013.83

013.84

013.85

013.86

013.90

013.91

013.92

013.93

013.94

013.95

013.96

014.00

014.01

014.02

014.03

014.04

014.05

014.06

014.80

014.81

014.82
CodeType

ICD-9 Diagnosis

ICD-9 Diagnosis

ICD-9 Diagnosis

ICD-9 Diagnosis

ICD-9 Diagnosis

ICD-9 Diagnosis

ICD-9 Diagnosis

ICD-9 Diagnosis

ICD-9 Diagnosis

ICD-9 Diagnosis

ICD-9 Diagnosis

ICD-9 Diagnosis

ICD-9 Diagnosis

ICD-9 Diagnosis

ICD-9 Diagnosis

ICD-9 Diagnosis

ICD-9 Diagnosis

ICD-9 Diagnosis

ICD-9 Diagnosis

ICD-9 Diagnosis

ICD-9 Diagnosis

ICD-9 Diagnosis

ICD-9 Diagnosis

ICD-9 Diagnosis

ICD-9 Diagnosis

ICD-9 Diagnosis

ICD-9 Diagnosis

ICD-9 Diagnosis

ICD-9 Diagnosis

ICD-9 Diagnosis

ICD-9 Diagnosis

ICD-9 Diagnosis

ICD-9 Diagnosis

ICD-9 Diagnosis

ICD-9 Diagnosis

ICD-9 Diagnosis

ICD-9 Diagnosis

ICD-9 Diagnosis

ICD-9 Diagnosis

ICD-9 Diagnosis

ICD-9 Diagnosis

ICD-9 Diagnosis

continued on next page 
APPENDIX B Code Lists (continued)

\section{Category}

Serious infection

Serious infection

Serious infection

Serious infection

Serious infection

Serious infection

Serious infection

Serious infection

Serious infection

Serious infection

Serious infection

Serious infection

Serious infection

Serious infection

Serious infection

Serious infection

Serious infection

Serious infection

Serious infection

Serious infection

Serious infection

Serious infection

Serious infection

Serious infection

Serious infection

Serious infection

Serious infection

Serious infection

Serious infection

Serious infection

Serious infection

Serious infection

Serious infection

Serious infection

Serious infection

Serious infection

Serious infection

Serious infection

Serious infection

Serious infection

Serious infection

Serious infection

\section{Description}

TB

TB

TB

TB

TB

TB

TB

TB

TB

TB

TB

TB

TB

TB

TB

TB

TB

TB

TB

TB

TB

TB

TB

TB

TB

TB

TB

TB

TB

TB

TB

TB

TB

TB

TB

TB

TB

TB

TB

TB

TB

TB

\section{Code}

014.83

014.84

014.85

014.86

015.00

015.01

015.02

015.03

015.04

015.05

015.06

015.10

015.11

015.12

015.13

015.14

015.15

015.16

015.20

015.21

015.22

015.23

015.24

015.25

015.26

015.50

015.51

015.52

015.53

015.54

015.55

015.56

015.60

015.61

015.62

015.63

015.64

015.65

015.66

015.70

015.71

015.72

\section{CodeType}

ICD-9 Diagnosis

ICD-9 Diagnosis

ICD-9 Diagnosis

ICD-9 Diagnosis

ICD-9 Diagnosis

ICD-9 Diagnosis

ICD-9 Diagnosis

ICD-9 Diagnosis

ICD-9 Diagnosis

ICD-9 Diagnosis

ICD-9 Diagnosis

ICD-9 Diagnosis

ICD-9 Diagnosis

ICD-9 Diagnosis

ICD-9 Diagnosis

ICD-9 Diagnosis

ICD-9 Diagnosis

ICD-9 Diagnosis

ICD-9 Diagnosis

ICD-9 Diagnosis

ICD-9 Diagnosis

ICD-9 Diagnosis

ICD-9 Diagnosis

ICD-9 Diagnosis

ICD-9 Diagnosis

ICD-9 Diagnosis

ICD-9 Diagnosis

ICD-9 Diagnosis

ICD-9 Diagnosis

ICD-9 Diagnosis

ICD-9 Diagnosis

ICD-9 Diagnosis

ICD-9 Diagnosis

ICD-9 Diagnosis

ICD-9 Diagnosis

ICD-9 Diagnosis

ICD-9 Diagnosis

ICD-9 Diagnosis

ICD-9 Diagnosis

ICD-9 Diagnosis

ICD-9 Diagnosis

ICD-9 Diagnosis

continued on next page 
APPENDIX B Code Lists (continued)

\section{Category}

Serious infection

Serious infection

Serious infection

Serious infection

Serious infection

Serious infection

Serious infection

Serious infection

Serious infection

Serious infection

Serious infection

Serious infection

Serious infection

Serious infection

Serious infection

Serious infection

Serious infection

Serious infection

Serious infection

Serious infection

Serious infection

Serious infection

Serious infection

Serious infection

Serious infection

Serious infection

Serious infection

Serious infection

Serious infection

Serious infection

Serious infection

Serious infection

Serious infection

Serious infection

Serious infection

Serious infection

Serious infection

Serious infection

Serious infection

Serious infection

Serious infection

Serious infection

\section{Description}

TB

TB

TB

TB

TB

TB

TB

TB

TB

TB

TB

TB

TB

TB

TB

TB

TB

TB

TB

TB

TB

TB

TB

TB

TB

TB

TB

TB

TB

TB

TB

TB

TB

TB

TB

TB

TB

TB

TB

TB

TB

TB

\section{Code}

015.73

015.74

015.75

015.76

015.80

015.81

015.82

015.83

015.84

015.85

015.86

015.90

015.91

015.92

015.93

015.94

015.95

015.96

016.00

016.01

016.02

016.03

016.04

016.05

016.06

016.10

016.11

016.12

016.13

016.14

016.15

016.16

016.20

016.21

016.22

016.23

016.24

016.25

016.26

016.30

016.31

016.32

\section{CodeType}

ICD-9 Diagnosis

ICD-9 Diagnosis

ICD-9 Diagnosis

ICD-9 Diagnosis

ICD-9 Diagnosis

ICD-9 Diagnosis

ICD-9 Diagnosis

ICD-9 Diagnosis

ICD-9 Diagnosis

ICD-9 Diagnosis

ICD-9 Diagnosis

ICD-9 Diagnosis

ICD-9 Diagnosis

ICD-9 Diagnosis

ICD-9 Diagnosis

ICD-9 Diagnosis

ICD-9 Diagnosis

ICD-9 Diagnosis

ICD-9 Diagnosis

ICD-9 Diagnosis

ICD-9 Diagnosis

ICD-9 Diagnosis

ICD-9 Diagnosis

ICD-9 Diagnosis

ICD-9 Diagnosis

ICD-9 Diagnosis

ICD-9 Diagnosis

ICD-9 Diagnosis

ICD-9 Diagnosis

ICD-9 Diagnosis

ICD-9 Diagnosis

ICD-9 Diagnosis

ICD-9 Diagnosis

ICD-9 Diagnosis

ICD-9 Diagnosis

ICD-9 Diagnosis

ICD-9 Diagnosis

ICD-9 Diagnosis

ICD-9 Diagnosis

ICD-9 Diagnosis

ICD-9 Diagnosis

ICD-9 Diagnosis

continued on next page 
APPENDIX B Code Lists (continued)

\section{Category}

Serious infection

Serious infection

Serious infection

Serious infection

Serious infection

Serious infection

Serious infection

Serious infection

Serious infection

Serious infection

Serious infection

Serious infection

Serious infection

Serious infection

Serious infection

Serious infection

Serious infection

Serious infection

Serious infection

Serious infection

Serious infection

Serious infection

Serious infection

Serious infection

Serious infection

Serious infection

Serious infection

Serious infection

Serious infection

Serious infection

Serious infection

Serious infection

Serious infection

Serious infection

Serious infection

Serious infection

Serious infection

Serious infection

Serious infection

Serious infection

Serious infection

Serious infection

\section{Description}

TB

TB

TB

TB

TB

TB

TB

TB

TB

TB

TB

TB

TB

TB

TB

TB

TB

TB

TB

TB

TB

TB

TB

TB

TB

TB

TB

TB

TB

TB

TB

TB

TB

TB

TB

TB

TB

TB

TB

TB

TB

TB

\section{Code}

016.33

016.34

016.35

016.36

016.40

016.41

016.42

016.43

016.44

016.45

016.46

016.50

016.51

016.52

016.53

016.54

016.55

016.56

016.60

016.61

016.62

016.63

016.64

016.65

016.66

016.70

016.71

016.72

016.73

016.74

016.75

016.76

016.90

016.91

016.92

016.93

016.94

016.95

016.96

017.00

017.01

017.02
CodeType

ICD-9 Diagnosis

ICD-9 Diagnosis

ICD-9 Diagnosis

ICD-9 Diagnosis

ICD-9 Diagnosis

ICD-9 Diagnosis

ICD-9 Diagnosis

ICD-9 Diagnosis

ICD-9 Diagnosis

ICD-9 Diagnosis

ICD-9 Diagnosis

ICD-9 Diagnosis

ICD-9 Diagnosis

ICD-9 Diagnosis

ICD-9 Diagnosis

ICD-9 Diagnosis

ICD-9 Diagnosis

ICD-9 Diagnosis

ICD-9 Diagnosis

ICD-9 Diagnosis

ICD-9 Diagnosis

ICD-9 Diagnosis

ICD-9 Diagnosis

ICD-9 Diagnosis

ICD-9 Diagnosis

ICD-9 Diagnosis

ICD-9 Diagnosis

ICD-9 Diagnosis

ICD-9 Diagnosis

ICD-9 Diagnosis

ICD-9 Diagnosis

ICD-9 Diagnosis

ICD-9 Diagnosis

ICD-9 Diagnosis

ICD-9 Diagnosis

ICD-9 Diagnosis

ICD-9 Diagnosis

ICD-9 Diagnosis

ICD-9 Diagnosis

ICD-9 Diagnosis

ICD-9 Diagnosis

ICD-9 Diagnosis

continued on next page 
APPENDIX B Code Lists (continued)

\section{Category}

Serious infection

Serious infection

Serious infection

Serious infection

Serious infection

Serious infection

Serious infection

Serious infection

Serious infection

Serious infection

Serious infection

Serious infection

Serious infection

Serious infection

Serious infection

Serious infection

Serious infection

Serious infection

Serious infection

Serious infection

Serious infection

Serious infection

Serious infection

Serious infection

Serious infection

Serious infection

Serious infection

Serious infection

Serious infection

Serious infection

Serious infection

Serious infection

Serious infection

Serious infection

Serious infection

Serious infection

Serious infection

Serious infection

Serious infection

Serious infection

Serious infection

Serious infection

\section{Description}

TB

TB

TB

TB

TB

TB

TB

TB

TB

TB

TB

TB

TB

TB

TB

TB

TB

TB

TB

TB

TB

TB

TB

TB

TB

TB

TB

TB

TB

TB

TB

TB

TB

TB

TB

TB

TB

TB

TB

TB

TB

TB

\section{Code}

017.03

017.04

017.05

017.06

017.10

017.11

017.12

017.13

017.14

017.15

017.16

017.20

017.21

017.22

017.23

017.24

017.25

017.26

017.30

017.31

017.32

017.33

017.34

017.35

017.36

017.40

017.41

017.42

017.43

017.44

017.45

017.46

017.50

017.51

017.52

017.53

017.54

017.55

017.56

017.60

017.61

017.62
CodeType

ICD-9 Diagnosis

ICD-9 Diagnosis

ICD-9 Diagnosis

ICD-9 Diagnosis

ICD-9 Diagnosis

ICD-9 Diagnosis

ICD-9 Diagnosis

ICD-9 Diagnosis

ICD-9 Diagnosis

ICD-9 Diagnosis

ICD-9 Diagnosis

ICD-9 Diagnosis

ICD-9 Diagnosis

ICD-9 Diagnosis

ICD-9 Diagnosis

ICD-9 Diagnosis

ICD-9 Diagnosis

ICD-9 Diagnosis

ICD-9 Diagnosis

ICD-9 Diagnosis

ICD-9 Diagnosis

ICD-9 Diagnosis

ICD-9 Diagnosis

ICD-9 Diagnosis

ICD-9 Diagnosis

ICD-9 Diagnosis

ICD-9 Diagnosis

ICD-9 Diagnosis

ICD-9 Diagnosis

ICD-9 Diagnosis

ICD-9 Diagnosis

ICD-9 Diagnosis

ICD-9 Diagnosis

ICD-9 Diagnosis

ICD-9 Diagnosis

ICD-9 Diagnosis

ICD-9 Diagnosis

ICD-9 Diagnosis

ICD-9 Diagnosis

ICD-9 Diagnosis

ICD-9 Diagnosis

ICD-9 Diagnosis

continued on next page 
APPENDIX B Code Lists (continued)

\section{Category}

Serious infection

Serious infection

Serious infection

Serious infection

Serious infection

Serious infection

Serious infection

Serious infection

Serious infection

Serious infection

Serious infection

Serious infection

Serious infection

Serious infection

Serious infection

Serious infection

Serious infection

Serious infection

Serious infection

Serious infection

Serious infection

Serious infection

Serious infection

Serious infection

Serious infection

Serious infection

Serious infection

Serious infection

Serious infection

Serious infection

Serious infection

Serious infection

Serious infection

Serious infection

Serious infection

Serious infection

Serious infection

Serious infection

Serious infection

Serious infection

Serious infection

Serious infection

\section{Description}

TB

TB

TB

TB

TB

TB

TB

TB

TB

TB

TB

TB

TB

TB

TB

TB

TB

TB

TB

TB

TB

TB

TB

TB

TB

TB

TB

TB

TB

TB

TB

TB

TB

TB

TB

TB

TB

TB

TB

TB

TB

TB

\section{Code}

017.63

017.64

017.65

017.66

017.70

017.71

017.72

017.73

017.74

017.75

017.76

017.80

017.81

017.82

017.83

017.84

017.85

017.86

017.90

017.91

017.92

017.93

017.94

017.95

017.96

018.00

018.01

018.02

018.03

018.04

018.05

018.06

018.80

018.81

018.82

018.83

018.84

018.85

018.86

018.90

018.91

018.92

\section{CodeType}

ICD-9 Diagnosis

ICD-9 Diagnosis

ICD-9 Diagnosis

ICD-9 Diagnosis

ICD-9 Diagnosis

ICD-9 Diagnosis

ICD-9 Diagnosis

ICD-9 Diagnosis

ICD-9 Diagnosis

ICD-9 Diagnosis

ICD-9 Diagnosis

ICD-9 Diagnosis

ICD-9 Diagnosis

ICD-9 Diagnosis

ICD-9 Diagnosis

ICD-9 Diagnosis

ICD-9 Diagnosis

ICD-9 Diagnosis

ICD-9 Diagnosis

ICD-9 Diagnosis

ICD-9 Diagnosis

ICD-9 Diagnosis

ICD-9 Diagnosis

ICD-9 Diagnosis

ICD-9 Diagnosis

ICD-9 Diagnosis

ICD-9 Diagnosis

ICD-9 Diagnosis

ICD-9 Diagnosis

ICD-9 Diagnosis

ICD-9 Diagnosis

ICD-9 Diagnosis

ICD-9 Diagnosis

ICD-9 Diagnosis

ICD-9 Diagnosis

ICD-9 Diagnosis

ICD-9 Diagnosis

ICD-9 Diagnosis

ICD-9 Diagnosis

ICD-9 Diagnosis

ICD-9 Diagnosis

ICD-9 Diagnosis

continued on next page 
APPENDIX B Code Lists (continued)

\section{Category}

Serious infection

Serious infection

Serious infection

Serious infection

Serious infection

Serious infection

Serious infection

Serious infection

Serious infection

Serious infection

Serious infection

Serious infection

Serious infection

Serious infection

Serious infection

Serious infection

Serious infection

Serious infection

Serious infection

Serious infection

Serious infection

Serious infection

Serious infection

Serious infection

Serious infection

Serious infection

Serious infection

Serious infection

Serious infection

Serious infection

Serious infection

Serious infection

Serious infection

Serious infection

Serious infection

Serious infection

Serious infection

Serious infection

Serious infection

Serious infection

Serious infection

Serious infection

\section{Description}

TB

TB

TB

TB

TB

$\mathrm{TB}$

TB

TB

TB

TB

Tonsillitis

Tonsillitis

Tonsillitis

Tonsillitis

Tonsillitis

Tonsillitis

Tonsillitis

Tonsillitis

Tonsillitis

Tonsillitis

Tonsillitis

Tonsillitis

Skin ulcer

Skin ulcer

Skin ulcer

Skin ulcer

Skin ulcer

Skin ulcer

Skin ulcer

Skin ulcer

Skin ulcer

Skin ulcer

Skin ulcer

Skin ulcer

Skin ulcer

Skin ulcer

Skin ulcer

Skin ulcer

Skin ulcer

Skin ulcer

Skin ulcer

Skin ulcer

\begin{tabular}{|c|c|}
\hline Code & CodeType \\
\hline 018.93 & ICD-9 Diagnosis \\
\hline 018.94 & ICD-9 Diagnosis \\
\hline 018.95 & ICD-9 Diagnosis \\
\hline 018.96 & ICD-9 Diagnosis \\
\hline 137.0 & ICD-9 Diagnosis \\
\hline 137.1 & ICD-9 Diagnosis \\
\hline 137.2 & ICD-9 Diagnosis \\
\hline 137.3 & ICD-9 Diagnosis \\
\hline 137.4 & ICD-9 Diagnosis \\
\hline V12.01 & ICD-9 Diagnosis \\
\hline 463 & ICD-9 Diagnosis \\
\hline 474.0 & ICD-9 Diagnosis \\
\hline 474.00 & ICD-9 Diagnosis \\
\hline 474.01 & ICD-9 Diagnosis \\
\hline 474.02 & ICD-9 Diagnosis \\
\hline 474.10 & ICD-9 Diagnosis \\
\hline 474.11 & ICD-9 Diagnosis \\
\hline 474.12 & ICD-9 Diagnosis \\
\hline 474.2 & ICD-9 Diagnosis \\
\hline 474.8 & ICD-9 Diagnosis \\
\hline 474.9 & ICD-9 Diagnosis \\
\hline 475 & ICD-9 Diagnosis \\
\hline 707.0 & ICD-9 Diagnosis \\
\hline 707.00 & ICD-9 Diagnosis \\
\hline 707.01 & ICD-9 Diagnosis \\
\hline 707.02 & ICD-9 Diagnosis \\
\hline 707.03 & ICD-9 Diagnosis \\
\hline 707.04 & ICD-9 Diagnosis \\
\hline 707.05 & ICD-9 Diagnosis \\
\hline 707.06 & ICD-9 Diagnosis \\
\hline 707.07 & ICD-9 Diagnosis \\
\hline 707.09 & ICD-9 Diagnosis \\
\hline 707.1 & ICD-9 Diagnosis \\
\hline 707.10 & ICD-9 Diagnosis \\
\hline 707.11 & ICD-9 Diagnosis \\
\hline 707.12 & ICD-9 Diagnosis \\
\hline 707.13 & ICD-9 Diagnosis \\
\hline 707.14 & ICD-9 Diagnosis \\
\hline 707.15 & ICD-9 Diagnosis \\
\hline 707.19 & ICD-9 Diagnosis \\
\hline 707.20 & ICD-9 Diagnosis \\
\hline 707.21 & ICD-9 Diagnosis \\
\hline
\end{tabular}


APPENDIX B Code Lists (continued)

\section{Category}

Serious infection

Serious infection

Serious infection

Serious infection

Serious infection

Serious infection

Serious infection

Serious infection

Serious infection

Serious infection

Serious infection

Serious infection

Serious infection

Serious infection

Serious infection

Serious infection

Serious infection

Serious infection

Serious infection

Serious infection

Serious infection

Serious infection

Serious infection

Serious infection

Serious infection

Serious infection

Serious infection

Serious infection

Serious infection

Serious infection

Serious infection

Serious infection

Serious infection

Serious infection

Serious infection

Serious infection

Serious infection

Serious infection

Serious infection

Serious infection

Serious infection

Serious infection

\section{Description}

Skin ulcer

Skin ulcer

Skin ulcer

Skin ulcer

Skin ulcer

Skin ulcer

Upper respiratory tract infection

Upper respiratory tract infection

Upper respiratory tract infection

Upper respiratory tract infection

Upper respiratory tract infection

Upper respiratory tract infection

Upper respiratory tract infection

Upper respiratory tract infection

Upper respiratory tract infection

Upper respiratory tract infection

Upper respiratory tract infection

Upper respiratory tract infection

Upper respiratory tract infection

Upper respiratory tract infection

Upper respiratory tract infection

Upper respiratory tract infection

Upper respiratory tract infection

Upper respiratory tract infection

Upper respiratory tract infection

Upper respiratory tract infection

Upper respiratory tract infection

Upper respiratory tract infection

Upper respiratory tract infection

Upper respiratory tract infection

Upper respiratory tract infection

Upper respiratory tract infection

Upper respiratory tract infection

Upper respiratory tract infection

Upper respiratory tract infection

Upper respiratory tract infection

Upper respiratory tract infection

Upper respiratory tract infection

Upper respiratory tract infection

Upper respiratory tract infection

Upper respiratory tract infection

Urinary tract infection

\section{Code}

707.22

707.23

707.24

707.25

707.8

707.9

032.0

032.1

032.2

032.3

034.0

460

461.0

461.1

461.2

461.3

461.8

461.9

462

464.0

464.00

464.01

464.10

464.11

464.20

464.21

464.30

464.31

464.4

464.50

464.51

465.0

465.8

465.9

473.0

473.1

473.2

473.3

473.8

473.9

784.91

032.84
CodeType

ICD-9 Diagnosis

ICD-9 Diagnosis

ICD-9 Diagnosis

ICD-9 Diagnosis

ICD-9 Diagnosis

ICD-9 Diagnosis

ICD-9 Diagnosis

ICD-9 Diagnosis

ICD-9 Diagnosis

ICD-9 Diagnosis

ICD-9 Diagnosis

ICD-9 Diagnosis

ICD-9 Diagnosis

ICD-9 Diagnosis

ICD-9 Diagnosis

ICD-9 Diagnosis

ICD-9 Diagnosis

ICD-9 Diagnosis

ICD-9 Diagnosis

ICD-9 Diagnosis

ICD-9 Diagnosis

ICD-9 Diagnosis

ICD-9 Diagnosis

ICD-9 Diagnosis

ICD-9 Diagnosis

ICD-9 Diagnosis

ICD-9 Diagnosis

ICD-9 Diagnosis

ICD-9 Diagnosis

ICD-9 Diagnosis

ICD-9 Diagnosis

ICD-9 Diagnosis

ICD-9 Diagnosis

ICD-9 Diagnosis

ICD-9 Diagnosis

ICD-9 Diagnosis

ICD-9 Diagnosis

ICD-9 Diagnosis

ICD-9 Diagnosis

ICD-9 Diagnosis

ICD-9 Diagnosis

ICD-9 Diagnosis

continued on next page 
APPENDIX B Code Lists (continued)

\section{Category}

Serious infection

Serious infection

Serious infection

Serious infection

Serious infection

Serious infection

Serious infection

Serious infection

Serious infection

Serious infection

Serious infection

Serious infection

Serious infection

Serious infection

Serious infection

Serious infection

Serious infection

Serious infection

Serious infection

Serious infection

Serious infection

Serious infection

Serious infection

Serious infection

Serious infection

Serious infection

Serious infection

Serious infection

Serious infection

Serious infection

Serious infection

Serious infection

Serious infection

Serious infection

Serious infection

Serious infection

Serious infection

Serious infection

Serious infection

Serious infection

Serious infection

Serious infection

\section{Description}

Urinary tract infection

Urinary tract infection

Urinary tract infection

Urinary tract infection

Urinary tract infection

Urinary tract infection

Urinary tract infection

Urinary tract infection

Urinary tract infection

Urinary tract infection

Urinary tract infection

Urinary tract infection

Urinary tract infection

Urinary tract infection

Urinary tract infection

Urinary tract infection

Urinary tract infection

Urinary tract infection

Urinary tract infection

Urinary tract infection

Urinary tract infection

Urinary tract infection

Urinary tract infection

Urinary tract infection

Urinary tract infection

Viral

Viral

Viral

Viral

Viral

Viral

Viral

Viral

Viral

Viral

Viral

Viral

Viral

Viral

Viral

Viral

Viral

\section{Code}

590.00

590.01

590.10

590.11

590.2

590.3

590.80

590.81

590.9

595.0

595.1

595.2

595.3

595.4

595.81

595.82

595.89

595.9

597.0

597.80

597.81

597.89

598.00

598.01

599.0

050.0

050.1

050.2

050.9

051.0

051.01

051.02

051.1

051.2

051.9

052.2

052.7

052.8

052.9

053.10

053.11

053.12
CodeType

ICD-9 Diagnosis

ICD-9 Diagnosis

ICD-9 Diagnosis

ICD-9 Diagnosis

ICD-9 Diagnosis

ICD-9 Diagnosis

ICD-9 Diagnosis

ICD-9 Diagnosis

ICD-9 Diagnosis

ICD-9 Diagnosis

ICD-9 Diagnosis

ICD-9 Diagnosis

ICD-9 Diagnosis

ICD-9 Diagnosis

ICD-9 Diagnosis

ICD-9 Diagnosis

ICD-9 Diagnosis

ICD-9 Diagnosis

ICD-9 Diagnosis

ICD-9 Diagnosis

ICD-9 Diagnosis

ICD-9 Diagnosis

ICD-9 Diagnosis

ICD-9 Diagnosis

ICD-9 Diagnosis

ICD-9 Diagnosis

ICD-9 Diagnosis

ICD-9 Diagnosis

ICD-9 Diagnosis

ICD-9 Diagnosis

ICD-9 Diagnosis

ICD-9 Diagnosis

ICD-9 Diagnosis

ICD-9 Diagnosis

ICD-9 Diagnosis

ICD-9 Diagnosis

ICD-9 Diagnosis

ICD-9 Diagnosis

ICD-9 Diagnosis

ICD-9 Diagnosis

ICD-9 Diagnosis

ICD-9 Diagnosis

continued on next page 
APPENDIX B Code Lists (continued)

\section{Category}

Serious infection

Serious infection

Serious infection

Serious infection

Serious infection

Serious infection

Serious infection

Serious infection

Serious infection

Serious infection

Serious infection

Serious infection

Serious infection

Serious infection

Serious infection

Serious infection

Serious infection

Serious infection

Serious infection

Serious infection

Serious infection

Serious infection

Serious infection

Serious infection

Serious infection

Serious infection

Serious infection

Serious infection

Serious infection

Serious infection

Serious infection

Serious infection

Serious infection

Serious infection

Serious infection

Serious infection

Serious infection

Serious infection

Serious infection

Serious infection

Serious infection

Serious infection

\section{Description}

Viral

Viral

Viral

Viral

Viral

Viral

Viral

Viral

Viral

Viral

Viral

Viral

Viral

Viral

Viral

Viral

Viral

Viral

Viral

Viral

Viral

Viral

Viral

Viral

Viral

Viral

Viral

Viral

Viral

Viral

Viral

Viral

Viral

Viral

Viral

Viral

Viral

Viral

Viral

Viral

Viral

Viral

\section{Code}

053.13

053.14

053.19

053.71

053.79

053.8

053.9

054.0

054.10

054.11

054.12

054.13

054.19

054.2

054.6

054.71

054.73

054.74

054.79

054.8

054.9

055.79

055.8

055.9

056.00

056.09

056.79

056.8

056.9

057.0

057.8

057.9

058.10

058.11

058.12

058.81

058.82

058.89

059.00

059.01

059.09

059.10

\section{CodeType}

ICD-9 Diagnosis

ICD-9 Diagnosis

ICD-9 Diagnosis

ICD-9 Diagnosis

ICD-9 Diagnosis

ICD-9 Diagnosis

ICD-9 Diagnosis

ICD-9 Diagnosis

ICD-9 Diagnosis

ICD-9 Diagnosis

ICD-9 Diagnosis

ICD-9 Diagnosis

ICD-9 Diagnosis

ICD-9 Diagnosis

ICD-9 Diagnosis

ICD-9 Diagnosis

ICD-9 Diagnosis

ICD-9 Diagnosis

ICD-9 Diagnosis

ICD-9 Diagnosis

ICD-9 Diagnosis

ICD-9 Diagnosis

ICD-9 Diagnosis

ICD-9 Diagnosis

ICD-9 Diagnosis

ICD-9 Diagnosis

ICD-9 Diagnosis

ICD-9 Diagnosis

ICD-9 Diagnosis

ICD-9 Diagnosis

ICD-9 Diagnosis

ICD-9 Diagnosis

ICD-9 Diagnosis

ICD-9 Diagnosis

ICD-9 Diagnosis

ICD-9 Diagnosis

ICD-9 Diagnosis

ICD-9 Diagnosis

ICD-9 Diagnosis

ICD-9 Diagnosis

ICD-9 Diagnosis

ICD-9 Diagnosis

continued on next page 
APPENDIX B Code Lists (continued)

\section{Category}

Serious infection

Serious infection

Serious infection

Serious infection

Serious infection

Serious infection

Serious infection

Serious infection

Serious infection

Serious infection

Serious infection

Serious infection

Serious infection

Serious infection

Serious infection

Serious infection

Serious infection

Serious infection

Serious infection

Serious infection

Serious infection

Serious infection

Serious infection

Serious infection

Serious infection

Serious infection

Serious infection

Serious infection

Serious infection

Serious infection

Serious infection

Serious infection

Serious infection

Serious infection

Serious infection

Serious infection

Serious infection

Serious infection

Serious infection

Serious infection

Serious infection

Serious infection

\section{Description}

Viral

Viral

Viral

Viral

Viral

Viral

Viral

Viral

Viral

Viral

Viral

Viral

Viral

Viral

Viral

Viral

Viral

Viral

Viral

Viral

Viral

Viral

Viral

Viral

Viral

Viral

Viral

Viral

Viral

Viral

Viral

Viral

Viral

Viral

Viral

Viral

Viral

Viral

Viral

Viral

Viral

Viral

\begin{tabular}{|c|c|}
\hline Code & CodeType \\
\hline 059.11 & ICD-9 Diagnosis \\
\hline 059.12 & ICD-9 Diagnosis \\
\hline 059.19 & ICD-9 Diagnosis \\
\hline 059.20 & ICD-9 Diagnosis \\
\hline 059.21 & ICD-9 Diagnosis \\
\hline 059.22 & ICD-9 Diagnosis \\
\hline 059.8 & ICD-9 Diagnosis \\
\hline 059.9 & ICD-9 Diagnosis \\
\hline 060.0 & ICD-9 Diagnosis \\
\hline 060.1 & ICD-9 Diagnosis \\
\hline 060.9 & ICD-9 Diagnosis \\
\hline 061 & ICD-9 Diagnosis \\
\hline 065.0 & ICD-9 Diagnosis \\
\hline 065.1 & ICD-9 Diagnosis \\
\hline 065.2 & ICD-9 Diagnosis \\
\hline 065.3 & ICD-9 Diagnosis \\
\hline 065.4 & ICD-9 Diagnosis \\
\hline 065.8 & ICD-9 Diagnosis \\
\hline 065.9 & ICD-9 Diagnosis \\
\hline 066.0 & ICD-9 Diagnosis \\
\hline 066.1 & ICD-9 Diagnosis \\
\hline 066.3 & ICD-9 Diagnosis \\
\hline 066.4 & ICD-9 Diagnosis \\
\hline 066.40 & ICD-9 Diagnosis \\
\hline 066.42 & ICD-9 Diagnosis \\
\hline 066. & $I C D$ \\
\hline 066.8 & ICD-9 Diagnosis \\
\hline 066.9 & ICD-9 Diagnosis \\
\hline 071 & ICD-9 Diagnosis \\
\hline 072.0 & ICD-9 Diagnosis \\
\hline 072.3 & ICD-9 Diagnosis \\
\hline 072.72 & ICD-9 Diagnosis \\
\hline 072.79 & ICD-9 Diagnosis \\
\hline 072.8 & ICD-9 Diagnosis \\
\hline 072.9 & ICD-9 Diagnosis \\
\hline 073.7 & ICD-9 Diagnosis \\
\hline 073.8 & ICD-9 Diagnosis \\
\hline 073.9 & ICD-9 Diagnosis \\
\hline 074.0 & ICD-9 Diagnosis \\
\hline 074.1 & ICD-9 Diagnosis \\
\hline 074.3 & ICD-9 Diagnosis \\
\hline 074.8 & ICD-9 Diagnosis \\
\hline
\end{tabular}

continued on next page 
APPENDIX B Code Lists (continued)

\section{Category}

Serious infection

Serious infection

Serious infection

Serious infection

Serious infection

Serious infection

Serious infection

Serious infection

Serious infection

Serious infection

Serious infection

Serious infection

Serious infection

Serious infection

Serious infection

Serious infection

Serious infection

Serious infection

Serious infection

Serious infection

Serious infection

Serious infection

Serious infection

Serious infection

Serious infection

Serious infection

Serious infection

Serious infection

Serious infection

Serious infection

Serious infection

Serious infection

Serious infection

Serious infection

Serious infection

Serious infection

\section{Description}

Viral

Viral

Viral

Viral

Viral

Viral

Viral

Viral

Viral

Viral

Viral

Viral

Viral

Viral

Viral

Viral

Viral

Viral

Viral

Viral

Viral

Viral

Viral

Viral

Viral

Viral

Viral

Viral

Viral

Viral

Viral

Viral

Viral

Viral

Viral

Viral

\section{Code}

075

078.0

078.1

078.10

078.11

078.12

078.19

078.2

078.4

078.5

078.6

078.7

078.81

078.82

078.88

078.89

079.0

079.1

079.2

079.3

079.4

079.50

079.51

079.52

079.59

079.6

079.8

079.81

079.82

079.83

079.88

079.89

079.9

079.98

079.99

790.8
CodeType

ICD-9 Diagnosis

ICD-9 Diagnosis

ICD-9 Diagnosis

ICD-9 Diagnosis

ICD-9 Diagnosis

ICD-9 Diagnosis

ICD-9 Diagnosis

ICD-9 Diagnosis

ICD-9 Diagnosis

ICD-9 Diagnosis

ICD-9 Diagnosis

ICD-9 Diagnosis

ICD-9 Diagnosis

ICD-9 Diagnosis

ICD-9 Diagnosis

ICD-9 Diagnosis

ICD-9 Diagnosis

ICD-9 Diagnosis

ICD-9 Diagnosis

ICD-9 Diagnosis

ICD-9 Diagnosis

ICD-9 Diagnosis

ICD-9 Diagnosis

ICD-9 Diagnosis

ICD-9 Diagnosis

ICD-9 Diagnosis

ICD-9 Diagnosis

ICD-9 Diagnosis

ICD-9 Diagnosis

ICD-9 Diagnosis

ICD-9 Diagnosis

ICD-9 Diagnosis

ICD-9 Diagnosis

ICD-9 Diagnosis

ICD-9 Diagnosis

ICD-9 Diagnosis 